\title{
EQUIVARIANT K-HOMOLOGY FOR HYPERBOLIC REFLECTION GROUPS
}

\author{
JEAN-FRANÇOIS LAFONT, IVONNE J. ORTIZ, ALEXANDER RAHM, \\ AND RUBÉN J. SÁNCHEZ-GARCÍA
}

\begin{abstract}
We compute the equivariant $K$-homology of the classifying space for proper actions, for cocompact 3-dimensional hyperbolic reflection groups. This coincides with the topological $K$-theory of the reduced $C^{*}$-algebra associated to the group, via the Baum-Connes conjecture. We show that, for any such reflection group, the associated $K$-theory groups are torsion-free. This means that we can complete previous computations with rational coefficients to get results with integral coefficients. On the way, we establish an efficient criterion for checking torsion-freeness of $K$-theory groups, which can be applied far beyond the scope of the present paper.
\end{abstract}

\section{INTRODUCTION}

For a discrete group $\Gamma$, a general problem is to compute $K_{*}\left(C_{r}^{*} \Gamma\right)$, the topological $K$-theory of the reduced $C^{*}$-algebra of $\Gamma$. The Baum-Connes Conjecture predicts that this functor can be determined, in a homological manner, from the complex representation rings of the finite subgroups of $\Gamma$. This viewpoint led to general recipes for computing the rational topological $K$-theory $K_{*}\left(C_{r}^{*} \Gamma\right) \otimes \mathbb{Q}$ of groups, through the use of Chern characters (see for instance Lück and Oliver [16] and Lück [13], [15], as well as related earlier work of Adem [1]). When $\Gamma$ has small homological dimension, one can sometimes even give completely explicit formulas for the rational topological $K$-theory, see for instance Lafont, Ortiz, and SánchezGarcía [11] for the case where $\Gamma$ is a 3-orbifold group.

On the other hand, performing integral calculations for these $K$-theory groups is much harder. For 2-dimensional crystallographic groups, such calculations have been done in M. Yang's thesis [25]. This was subsequently extended to the class of cocompact planar groups by Lück and Stamm [18], and to certain higher dimensional dimensional crystallographic groups by Davis and Lück [4] (see also Langer and Lück [12]). For 3-dimensional groups, Lück [14] completed this calculation for the semi-direct product $\mathrm{Hei}_{3}(\mathbb{Z}) \rtimes \mathbb{Z}_{4}$ of the 3 -dimensional integral Heisenberg group with a specific action of the cyclic group $\mathbb{Z}_{4}$. Some further computations were completed by Isely [7] for groups of the form $\mathbb{Z}^{2} \rtimes \mathbb{Z}$; by Rahm [22] for the class of Bianchi groups; by Pooya and Valette [21] for solvable Baumslag-Solitar groups; and by Flores, Pooya and Valette [5] for lamplighter groups of finite groups.

When the result was that the $K$-theory groups are torsion-free, like in the above quoted papers [14] and [4], then so far, this was considered as an ad-hoc computational result. In the present paper, we can however explain such lack of torsion with a new criterion (Theorem 4), which can be checked very efficiently (we do this

Date: October 25, 2017. 
for the Heisenberg semidirect product group of [14] in Appendix C, and for the crystallographic groups of [4] in Appendix D).

The main purpose of the present paper is to establish the following formula for the integral $K$-theory groups of cocompact 3-dimensional hyperbolic reflection groups. We note that, by a celebrated result of Andre'ev [2], there is a simple algorithm that inputs a Coxeter group $\Gamma$, and decides whether or not there exists a hyperbolic polyhedron $P_{\Gamma} \subset \mathbb{H}^{3}$ which generates $\Gamma$. In particular, given an arbitrary Coxeter group, one can easily verify if it is covered by the following Main Theorem.

Main Theorem. Let $\Gamma$ be a cocompact 3-dimensional hyperbolic reflection group, generated by reflections in the side of a hyperbolic polyhedron $\mathcal{P} \subset \mathbb{H}^{3}$. Then

$$
K_{0}\left(C_{r}^{*}(\Gamma)\right) \cong \mathbb{Z}^{c f(\Gamma)} \text { and } K_{1}\left(C_{r}^{*}(\Gamma)\right) \cong \mathbb{Z}^{c f(\Gamma)-\chi(\mathcal{C})},
$$

where the integers $c f(\Gamma), \chi(\mathcal{C})$ can be explicitly computed from the combinatorics of the polyhedron $\mathcal{P}$.

Let us briefly describe the contents of the paper. In Section 2, we provide background material on hyperbolic reflection groups, topological $K$-theory, and the Baum-Connes Conjecture. We also introduce our main tool, the Atiyah-Hirzebruch type spectral sequence. In Section 3, we use the spectral sequence to show that the $K$-theory groups we are interested in coincide with the Bredon homology groups $\mathrm{H}_{0}^{\mathfrak{F i n}}\left(\Gamma ; R_{\mathbb{C}}\right)$ and $\mathrm{H}_{1}^{\mathfrak{F i n}}\left(\Gamma ; R_{\mathbb{C}}\right)$ respectively. We also explain, using the $\Gamma$-action on $\mathbb{H}^{3}$, why the homology group $\mathrm{H}_{1}^{\mathfrak{乛 i n}}\left(\Gamma ; R_{\mathbb{C}}\right)$ is torsion-free. In contrast, showing that $\mathrm{H}_{0}^{\mathfrak{F i n}}\left(\Gamma ; R_{\mathbb{C}}\right)$ is torsion-free is much more difficult. In Section 4 , we give a linear algebraic proof, inspired by the "representation ring splitting" technique of [22], and establishing a novel criterion for proving torsion-freedom of $\mathrm{H}_{0}^{\mathfrak{F i n}}\left(\Gamma ; R_{\mathbb{C}}\right)$ for any collection of groups $\Gamma$ with specified types of finite subgroups. Note that our proven lack of torsion in the Bredon homology is not a property shared by all discrete groups acting on hyperbolic 3-space: for example, 2-torsion occurs in $\mathrm{H}_{0}^{\mathfrak{F i n}}\left(\Gamma ; R_{\mathbb{C}}\right)$ for a Bianchi group $\Gamma$ whenever it has a 2-dihedral subgroup $C_{2} \times C_{2}$ [22].

Finally, in Section 5, we provide an explicit formula for the rank of the Bredon homology groups (and hence for the $K$-groups we are interested in), in terms of the combinatorics of the polyhedron $\mathcal{P}$. Our paper concludes with two fairly long Appendices, which contain all the character tables and induction homomorphisms used in our proofs, making our results explicit and self-contained.

\section{Funding}

Portions of this work were carried out during multiple collaborative visits at Ohio State University, Miami University, and the University of Southampton. The authors would like to thank these institutions for their hospitality. Lafont was partly supported by the NSF, under grant DMS-1510640. Ortiz was partly supported by the NSF, under grant DMS-1207712. Rahm was supported by Gabor Wiese's University of Luxembourg grant AMFOR.

\section{BACKGROUND MATERIAL}

2.1. $K$-theory and the Baum-Connes Conjecture. Associated to a discrete group $\Gamma$, one has $C_{r}^{*} \Gamma$, the reduced $C^{*}$-algebra of $\Gamma$. This algebra is defined to be the closure, in the operator norm, of the linear span of the image of the regular representation $\lambda: \Gamma \rightarrow B\left(l^{2}(\Gamma)\right)$ of $\Gamma$ on the Hilbert space $l^{2}(\Gamma)$ of square-summable 
complex valued functions on $\Gamma$. This algebra encodes various analytic properties of the group $\Gamma[20]$.

For a $C^{*}$-algebra $A$, one can define the topological $K$-theory groups $K_{*}(A):=$ $\pi_{*-1}(G L(A))$, which are the homotopy groups of the space $G L(A)$ of invertible matrices with entries in $A$. Due to Bott periodicity, there are canonical isomorphisms $K_{*}(A) \cong K_{*+2}(A)$, and thus it is sufficient to consider $K_{0}(A)$ and $K_{1}(A)$.

In the special case where $A=C_{r}^{*} \Gamma$, the Baum-Connes Conjecture predicts that there is a canonical isomorphism $K_{n}^{\Gamma}(X) \rightarrow K_{n}\left(C_{r}^{*}(\Gamma)\right)$, where $X$ is a model for $\underline{E} \Gamma$ (the classifying space for $\Gamma$-actions with isotropy in the family of finite subgroups), and $K_{*}^{\Gamma}(-)$ is the equivariant $K$-homology functor. The Baum-Connes conjecture has been verified for many classes of groups. We refer the interested reader to the monograph by Mislin and Valette [20] or the survey article by Lück and Reich [17] for more information about these topics.

2.2. Hyperbolic reflection groups. By a $d$-dimensional hyperbolic polyhedron, we mean the region of $\mathbb{H}^{d}$ enclosed by a given finite number of (geodesic) hyperplanes, that is, the intersection of a collection of half-spaces associated to the hyperplanes. Let $\mathcal{P} \subset \mathbb{H}^{d}$ be a polyhedron such that all the interior angles between faces are of the form $\pi / m_{i j}$ where $m_{i j} \geq 2$ an integer (although some pairs of faces may be disjoint). Let $\Gamma=\Gamma_{\mathcal{P}}$ the associated Coxeter group generated by the reflections in the hyperplanes containing the faces of $\mathcal{P}$.

The $\Gamma$-space $\mathbb{H}^{d}$ is then a model of $\underline{E} \Gamma$ with fundamental domain $\mathcal{P}$. This is a strict fundamental domain, that is, no further points of $\mathcal{P}$ are identified under the group action, and hence $\mathcal{P}=\Gamma \backslash \mathbb{H}^{d}$ (we will use left-action notation). Recall that $\Gamma$ admits the following Coxeter presentation

$$
\Gamma=\left\langle s_{1}, \ldots, s_{n} \mid\left(s_{i} s_{j}\right)^{m_{i j}}\right\rangle
$$

where $n$ is the number of distinct hyperplanes enclosing $\mathcal{P}, s_{i}$ denotes the reflection on the $i^{\text {th }}$ face, and $m_{i j} \geq 2$ are integers such that: $m_{i i}=1$ for all $i$, and, if $i \neq j$, the corresponding faces meet with interior angle $\pi / m_{i j}$. We will write $m_{i j}=\infty$ if the corresponding faces do not intersect.

Note that $\mathcal{P}$ may not be a $\mathrm{CW}$ or simplicial complex with the natural structure given by vertices, edges, faces, etc (for example, an ideal triangle in $\mathbb{H}^{2}$ or the region enclosed by two distinct hyperplanes). However, if $\mathcal{P}$ has finite volume, it is a simplicial complex except from possibly ideal vertices at infinity. In such a case (i.e. $\mathcal{P}$ non-compact) we can obtain a cocompact model of $\underline{E} \Gamma$ by equivariantly removing some horoballs in $\mathbb{H}^{d}$ (see $\S 4$ in [8]). Hence a fundamental domain of the resulting action would be a copy of $\mathcal{P}$ with all ideal vertices truncated.

For the rest of this article, $d=3, \mathcal{P}$ is compact, and $X$ is $\mathbb{H}^{3}$ with the $\Gamma$-action described above.

2.3. Cell structure of the orbit space. Let $J=\{1, \ldots, n\}$ and write $\langle S\rangle$ for the subgroup generated by a subset $S \subset \Gamma$. At a vertex of $\mathcal{P}$, the concurrent faces (a minimum of 3) must generate a reflection group acting on the 2 -sphere, hence it must be a spherical triangle group and in particular it forces the number of incident faces to be exactly three. The only finite Coxeter groups with 3 generators are the triangle groups $\Delta(2,2, m)$ for some $m \geq 2, \Delta(2,3,3), \Delta(2,3,4)$ and $\Delta(2,3,5)$, where we use the notation

$$
\Delta(p, q, r)=\left\langle s_{1}, s_{2}, s_{3} \mid s_{1}^{2}, s_{2}^{2}, s_{3}^{2},\left(s_{1} s_{2}\right)^{p},\left(s_{1} s_{3}\right)^{q},\left(s_{2} s_{3}\right)^{r}\right\rangle .
$$


Starting from our compact polyhedron $\mathcal{P}$, we obtain a $\Gamma$-CW-structure on $X=\mathbb{H}^{3}$ with:

- one orbit of 3-cells, with trivial stabilizer;

- $n$ orbits of 2 -cells (faces) with stabilisers $\left\langle s_{i}\right\rangle \cong \mathbb{Z} / 2(i=1, \ldots, n)$;

- one orbit of 1-cells (edges) per (unordered) pair $i, j \in J$ with $m_{i j} \neq \infty$, with stabilizer $\left\langle s_{i}, s_{j}\right\rangle \cong D_{m_{i j}}$ dihedral;

- one orbit of 0-cells (vertices) per (unordered) triple $i, j, k \in J$ with $\left\langle s_{i}, s_{j}, s_{k}\right\rangle$ finite, with stabilizer $\left\langle s_{i}, s_{j}, s_{k}\right\rangle \cong \Delta\left(m_{i j}, m_{i k}, m_{j k}\right)$.

We introduce the following notation (after having fixed an order on the Coxeter generators, or equivalently, on the faces of the polyhedron) for the simplices of $\mathcal{P}$ :

$$
\begin{aligned}
& f_{i} \text { (faces), } \\
& e_{i j}=f_{i} \cap f_{j} \quad \text { (edges), } \\
& v_{i j k}=f_{i} \cap f_{j} \cap f_{k}=e_{i j} \cap e_{i k} \cap e_{j k} \quad \text { (vertices), }
\end{aligned}
$$

whenever the intersections are non-empty, that is, whenever $m_{i j} \neq \infty$, respectively when $\left\{m_{i j}, m_{i k}, m_{j k}\right\}$ equals $\{2,2, m\}$ for some $m \geq 2,\{2,3,3\},\{2,3,4\}$ or $\{2,3,5\}$.

2.4. A spectral sequence. We ultimately want to compute the $K$-theory groups of the reduced $C^{*}$-algebra of $\Gamma$ via the Baum-Connes conjecture. Note that the conjecture holds for these groups: Coxeter groups have the Haagerup property [3] and hence satisfy Baum-Connes [6]. Therefore, it suffices to compute the equivariant $K$-homology groups $K_{*}^{\Gamma}(X)$, since $X$ is a model of $\underline{E} \Gamma$. In turn, these groups can be obtained by the Bredon homology of $X$ and an equivariant Atiyah-Hirezebruch spectral sequence coming from the inclusion of the skeleta of the $\Gamma-\mathrm{CW}$-complex $X$ [19]. The second page of this spectral sequence is given by the Bredon homology groups (cf. [19])

$$
E_{p, q}^{2}= \begin{cases}\mathrm{H}_{p}^{\mathfrak{F i n}}\left(\Gamma ; R_{\mathbb{C}}\right) & q \text { even } \\ 0 & q \text { odd }\end{cases}
$$

The coefficients of the Bredon homology groups (a functor from the orbit category with respect to the family of finite subgroups of $\Gamma$, to $\mathbb{Z}$-modules) are given by the complex representation ring of the cell stabilizers, which are finite subgroups. In order to simplify notation, we will often write $H_{p}$ to denote $\mathrm{H}_{p}^{\mathfrak{F} \mathfrak{i n}}\left(\Gamma ; R_{\mathbb{C}}\right)$.

Before defining Bredon homology, we note that $\operatorname{dim}(X)=3$ already implies, by homological algebra arguments, the following.

Proposition 1. There are short exact sequences

$$
0 \longrightarrow \operatorname{coker}\left(d_{3,0}^{3}\right) \longrightarrow K_{0}^{\Gamma}(X) \longrightarrow H_{2} \longrightarrow 0
$$

and

$$
0 \longrightarrow H_{1} \longrightarrow K_{1}^{\Gamma}(X) \longrightarrow \operatorname{ker}\left(d_{3,0}^{3}\right) \longrightarrow 0 \text {. }
$$

(Here $d_{3,0}^{3}: E_{3,0}^{3}=H_{3} \longrightarrow E_{0,2}^{3}=H_{2}$ is the differential on the $E^{3}$-page.)

Proof. This follows at once from [19, Theorem 5.29] but, for completeness, we give a direct proof based on the Atiyah-Hirzebruch spectral sequence. Write $K_{n}$ for $K_{n}^{\Gamma}(X)$. Firstly, note that the $E^{2}$-page is concentrated in the $0 \leq p \leq 3$ columns (since $\operatorname{dim}(X) \leq 3$ ), and in the $q$ even rows - see (4) above. 
Secondly, recall that the bidegree of the differentials $d_{p, q}^{k}$ on the $E^{k}$-page is $(-k, k-1)$. Thus the only non-trivial differentials are $d_{3, q}^{3}, q \geq 0$ even, from $E_{3, q}^{3}=$ $H_{3}$ to $E_{0, q+2}^{3}=H_{0}\left(\right.$ since $\left.E^{3}=E^{2}\right)$. All in all, $E^{\infty}=E^{2}$ except $E_{3, q}^{\infty}=\operatorname{ker}\left(d_{3, q}^{3}\right)$ and $E_{0, q}^{\infty}=\operatorname{coker}\left(d_{3, q}^{3}\right)$, that is,

$$
E_{p, q}^{\infty}= \begin{cases}H_{p} & p=1,2 \text { and } q \text { even, } \\ \operatorname{coker}\left(d_{3,0}^{3}\right) & p=0 \text { and } q \text { even, } \\ \operatorname{ker}\left(d_{3,0}^{3}\right) & p=3 \text { and } q \text { even, } \\ 0 & \text { otherwise. }\end{cases}
$$

Now consider the filtration $\ldots \subset F_{p-1, q+1} \subset F_{p, q} \subset \ldots$ of $K_{p+q}$, where each $F_{p, q}=\operatorname{Im}\left(K_{p+q}^{\Gamma}\left(X^{p}\right) \rightarrow K_{p+q}^{\Gamma}(X)\right)$, the image of the map induced in $K$-homology by the inclusion of the $p$-skeleton in $X$. In particular, $F_{p, q}=0$ for $p<0$ and $F_{p, q}=K_{p+q}$ for $p \geq \operatorname{dim}(X)=3$. The quotient $F_{p, q} / F_{p-1, q+1}$ is isomorphic to $E_{p, q}^{\infty}=E_{p, q}^{2}$, as given above. Therefore we have, on one hand, the filtration:

$$
0=F_{-1,2}=F_{0,1} \subset F_{1,0}=F_{2,-1} \subset F_{3,-2}=K_{1},
$$

with only two non-trivial quotients isomorphic to $E_{1,0}^{\infty}=H_{1}$ and $E_{3,-2}^{\infty}=\operatorname{ker}\left(d_{3,0}^{3}\right)$. On the other hand, we have the filtration:

$$
0=F_{-1,1} \subset F_{0,0}=F_{1,-1} \subset F_{2,-2}=F_{3,-3}=K_{0},
$$

with non-trivial quotients isomorphic to $E_{0,0}^{\infty}=\operatorname{coker}\left(d_{3,0}^{3}\right)$ and $E_{2,-2}^{\infty}=H_{2}$. These results combined together give the short exact sequences above.

2.5. Bredon Homology. To shorten notation, write $\Gamma_{e}$ for $\operatorname{stab}_{\Gamma}(e)$. The Bredon homology groups in (4) can be defined as the homology groups of the following chain complex (recall that $X$ is a model of $\underline{E} \Gamma$ )

$$
\ldots \longrightarrow \bigoplus_{e \in I_{d}} R_{\mathbb{C}}\left(\Gamma_{e}\right) \stackrel{\partial_{d}}{\longrightarrow} \bigoplus_{e \in I_{d-1}} R_{\mathbb{C}}\left(\Gamma_{e}\right) \longrightarrow \ldots,
$$

where $I_{d}$ is a set of orbit representatives of $d$-cells $(d \geq 0)$, and $\partial_{d}$ is defined via the geometric boundary map and induction between representation rings, as follows. If $g e^{\prime}$ is in the boundary of $e\left(e \in I_{d}, e^{\prime} \in I_{d-1}, g \in \Gamma\right)$, then $\partial$ restricted to $R_{\mathbb{C}}\left(\Gamma_{e}\right) \rightarrow R_{\mathbb{C}}\left(\Gamma_{e^{\prime}}\right)$ is given by the composition

$$
R_{\mathbb{C}}\left(\Gamma_{e}\right) \stackrel{\text { ind }}{\longrightarrow} R_{\mathbb{C}}\left(\Gamma_{g e^{\prime}}\right) \stackrel{\cong}{\longrightarrow} R_{\mathbb{C}}\left(\Gamma_{e^{\prime}}\right),
$$

where the first map is the induction homomorphism of representation rings associated to the subgroup inclusion $\Gamma_{e} \subset \Gamma_{g e^{\prime}}$, and the second is the isomorphism induced by conjugation $\Gamma_{g e^{\prime}}=g \Gamma_{e^{\prime}} g^{-1}$. Finally, we add a sign depending on a chosen (and thereafter fixed) orientation on the faces of $\mathcal{P}$. The value $\partial_{d}(e)$ equals the sum of these maps over all boundary cells of $e$.

Since $\mathcal{P}$ is a strict fundamental domain, we can choose the faces of $\mathcal{P}$ as orbit representatives and thus $g$ (as above) is always the identity. We will implicitly make this assumption from now on.

\section{Analyzing the Bredon chain complex for $\Gamma$}

Let $S=\left\{s_{i}: 1 \leq i \leq n\right\}$ be the set of Coxeter generators and $J=\{1, \ldots, n\}$. Let

$$
0 \longrightarrow \mathcal{C}_{3} \stackrel{\partial_{3}}{\longrightarrow} \mathcal{C}_{2} \stackrel{\partial_{2}}{\longrightarrow} \mathcal{C}_{1} \stackrel{\partial_{1}}{\longrightarrow} \mathcal{C}_{0} \longrightarrow 0
$$


be the Bredon chain complex associated to $X$ (since $X$ is 3-dimensional).

Next we analyse each differential in the chain complex above. Recall that for a finite group $G$ the complex representation ring $R_{\mathbb{C}}(G)$ is defined as the free abelian group with basis the set of irreducible representations of $G$ (the ring structure is not relevant in this setting). Hence $R_{\mathbb{C}}(G) \cong \mathbb{Z}^{c(G)}$, where we write $c(G)$ for the set of conjugacy classes in $G$.

3.1. Analysis of $\partial_{3}$. Let $G$ be a finite group with irreducible representations $\rho_{1}, \ldots, \rho_{m}$ of degree $n_{1}, \ldots, n_{m}$, and $\tau$ the only representation of the trivial subgroup $\left\{1_{G}\right\} \leq G$. Then $\tau$ induces the regular representation in $G$ :

$$
\operatorname{Ind}_{\left\{1_{G}\right\}}^{G}(\tau)=n_{1} \rho_{1}+\ldots+n_{m} \rho_{m} .
$$

Lemma 1. Let $X$ be a $\Gamma-C W$-complex with finite stabilizers, and $k \in \mathbb{N}$. If there is a unique orbit of $k$-cells and this orbit has trivial stabiliser, then $H_{k}=0$, provided that $\partial_{k} \neq 0$.

Proof. The Bredon module $\mathcal{C}_{k}$ equals $R_{\mathbb{C}}(\langle 1\rangle) \cong \mathbb{Z}$ with generator $\tau$, the trivial representation. Then $\partial_{k}(\tau) \neq 0$ implies $\operatorname{ker}\left(\partial_{k}\right)=0$ and therefore the corresponding homology group vanishes.

From the lemma we immediately conclude that $H_{3}=0$ if $\partial_{3} \neq 0$. The case $\partial_{3}=0$ occurs if and only if all boundary faces are pairwise identified, which cannot happen since $\mathcal{P}$ is a strict fundamental domain (the group acts by reflections on the faces). We conclude that $H_{3}=0$ and, using Proposition 1, we obtain:

Proposition 2. We have $K_{1}^{\Gamma}(X) \cong H_{1}$, and there is a short exact sequence

$$
0 \longrightarrow H_{0} \longrightarrow K_{0}^{\Gamma}(X) \longrightarrow H_{2} \longrightarrow 0
$$

3.2. Analysis of $\partial_{2}$. Let $f$ be a face of $\mathcal{P}$ and $e \in \partial f$ an edge. Suppose, using the notation in (3), that $f=f_{i}$ and $e=e_{i j}$. Then we have a map $R_{\mathbb{C}}\left(\left\langle s_{i}\right\rangle\right) \rightarrow$ $R_{\mathbb{C}}\left(\left\langle s_{i}, s_{j}\right\rangle\right)$ induced by inclusion. Recall that $\left\langle s_{i}\right\rangle \cong C_{2}$ and $\left\langle s_{i}, s_{j}\right\rangle \cong D_{m_{i j}}$. A straightforward analysis (see Appendix A for character tables, and Appendix B for induction homomorphism notation and calculations) shows that

if $i<j$, or

$$
\begin{aligned}
& \rho_{1} \uparrow=\chi_{1}+\widehat{\chi_{4}}+\sum \phi_{p}, \\
& \rho_{2} \uparrow=\chi_{2}+\widehat{\chi_{3}}+\sum \phi_{p},
\end{aligned}
$$

$$
\begin{aligned}
& \rho_{1} \uparrow=\chi_{1}+\widehat{\chi_{3}}+\sum \phi_{p}, \\
& \rho_{2} \uparrow=\chi_{2}+\widehat{\chi_{4}}+\sum \phi_{p},
\end{aligned}
$$

if $j<i$. That is, as a map of free abelian groups $\mathbb{Z}^{2} \rightarrow \mathbb{Z}^{c\left(D_{m_{i j}}\right)}$,

$$
\begin{aligned}
& (a, b) \mapsto \pm(a, b, \widehat{b}, \widehat{a}, a+b, \ldots, a+b) \quad \text { or } \\
& (a, b) \mapsto \pm(a, b, \widehat{a}, \widehat{b}, a+b, \ldots, a+b),
\end{aligned}
$$

Using the analysis above, we can now show

Theorem 1. For any compact $\mathcal{P}$, we have that $H_{2}=0$.

From this theorem and Proposition 2, we immediately obtain 
Corollary 1. $K_{0}^{\Gamma}(X)=H_{0}$ and $K_{1}^{\Gamma}(X)=H_{1}$.

Proof of Theorem. Fix an orientation on the polyhedron $\mathcal{P}$, and consider the induced orientations on the faces. At an edge we have two incident faces $f_{i}$ and $f_{j}$ with opposite orientations so without loss of generality we have, as a map of free abelian groups,

$$
\begin{aligned}
R_{\mathbb{C}}\left(\left\langle s_{i}\right\rangle\right) \oplus R_{\mathbb{C}}\left(\left\langle s_{j}\right\rangle\right) \cong \mathbb{Z}^{2} \oplus \mathbb{Z}^{2} & \rightarrow \mathbb{Z}^{c\left(D_{m_{i j}}\right)} \cong R_{\mathbb{C}}\left(\left\langle s_{i}, s_{j}\right\rangle\right) \\
(a, b \mid c, d) & \mapsto(a-c, b-d, \widehat{a-d}, \widehat{b-c}, S, \ldots, S)
\end{aligned}
$$

where $S=a+b-c-d$, and the elements with a hat ${ }^{-}$appear only when $m_{i j}$ is even. Note that we use vertical bars '|' for clarity, to separate elements coming from different representation rings.

By the preceding analysis, $\partial_{2}(x)=0$ implies that, for each $i, j \in J$, if the faces $f_{i}$ and $f_{j}$ meet, then

(1) $a_{i}=a_{j}$ and $b_{i}=b_{j}$, if $m_{i j}$ is odd, and

(2) $a_{i}=a_{j}=b_{i}=b_{j}$, if $m_{i j}$ is even.

Suppose that $f_{1}, \ldots, f_{n}$ are the faces of $\mathcal{P}$. Let $x=\left(a_{1}, b_{1}|\ldots| a_{n}, b_{n}\right) \in \mathcal{C}_{2}$ be an element in $\operatorname{Ker}\left(\partial_{2}\right)$. Note that $\partial \mathcal{P}$ is connected (since $P$ is homeomorphic to $\mathbb{D}^{3}$ ), so by (1) and (2) above, we have that $a_{1}=\ldots=a_{n}$ and $b_{1}=\ldots=b_{n}$. Since the stabilizer of a vertex is a spherical triangle group, there is an even $m_{i j}$, which also forces $a=b$. Therefore we have $x=(a, a|\ldots| a, a)$ so $x=\partial_{3}(a)$ (note that the choice of orientation above forces all signs to be positive), and this gives $\operatorname{ker}\left(\partial_{2}\right) \subseteq \operatorname{im}\left(\partial_{3}\right)$, which suffices to prove equality and hence the vanishing of the second homology group.

3.3. Analysis of $\partial_{1}$. Let $e=e_{i j}$ be an edge and $v=v_{i j k} \in \partial e$ a vertex, using the notation in (3). We study all possible induction homomorphisms $R_{\mathbb{C}}\left(\left\langle s_{i}, s_{j}\right\rangle\right) \rightarrow$ $R_{\mathbb{C}}\left(\left\langle s_{i}, s_{j}, s_{k}\right\rangle\right)$ in Appendix B and conclude that $H_{1}$ is torsion-free, as follows.

Theorem 2. There is no torsion in $H_{1}$.

Proof. Consider the Bredon chain complex

$$
\mathcal{C}_{2} \stackrel{\partial_{2}}{\longrightarrow} \mathcal{C}_{1} \stackrel{\partial_{1}}{\longrightarrow} \mathcal{C}_{0}
$$

To prove that $H_{1}=\operatorname{ker}\left(\partial_{1}\right) / \operatorname{im}\left(\partial_{2}\right)$ is torsion-free, it suffices to prove that $\mathcal{C}_{1} / \operatorname{im}\left(\partial_{2}\right)$ is torsion-free. Let $\alpha \in \mathcal{C}_{1}$ and $0 \neq k \in \mathbb{Z}$ such that $k \alpha \in \operatorname{im}\left(\partial_{2}\right)$. We shall prove that $\alpha \in \operatorname{im}\left(\partial_{2}\right)$.

Since $k \alpha \in \operatorname{im}\left(\partial_{2}\right)$, we can find $\beta \in \mathcal{C}_{2}$ with $\partial_{2}(\beta)=k \alpha$. Suppose that $\mathcal{P}$ has $n$ faces, and write $\beta=\left(a_{1}, b_{1}|\ldots| a_{n}, b_{n}\right) \in \mathcal{C}_{2}$, using vertical bars '|' to separate elements coming from different representation rings. We shall see that one can find a 1-chain $\beta^{\prime}$, homologous to $\beta$, and with every entry of $\beta^{\prime}$ a multiple of $k$.

At an edge $e_{i j}$, the differential $\partial_{2}$ takes the form (cf. $\S 3.2$ )

$$
\begin{aligned}
& R_{\mathbb{C}}\left(\left\langle s_{i}\right\rangle\right) \oplus R_{\mathbb{C}}\left(\left\langle s_{j}\right\rangle\right) \longrightarrow R_{\mathbb{C}}\left(\left\langle s_{i}, s_{j}\right\rangle\right) \\
& \left(a, b \mid a^{\prime}, b^{\prime}\right) \quad \mapsto \quad \pm\left(a-a^{\prime}, b-b^{\prime}, \widehat{b-a^{\prime}}, \widehat{a-b^{\prime}} \mid S, \ldots, S\right),
\end{aligned}
$$

where $S=a+b-a^{\prime}-b^{\prime}$, and the elements with a hat ${ }^{\wedge}$ appearing only when $m_{i j}$ is even. Since every entry of $\partial_{2}(\beta)$ is a multiple of $k$, using (9), we have that for every pair of intersecting faces $f_{i}$ and $f_{j}$,

$$
a_{i} \equiv a_{j} \quad(\bmod k) \quad \text { and } \quad b_{i} \equiv b_{j} \quad(\bmod k) .
$$


Equation (9) also shows that $\mathbf{1}_{\partial P}=(1,1|\ldots| 1,1)$, the formal sum over all generators of representation rings of face stabilizers of $\partial P \in \mathcal{C}_{2}$, is in the kernel of $\partial_{2}$. In particular, setting $\beta^{\prime}=\beta-a_{1} \mathbf{1}_{\partial P}$, we see that $\partial_{2}\left(\beta^{\prime}\right)=\partial_{2}(\beta)=\alpha$ and we can assume without loss of generality that $\beta^{\prime}$ satisfies $a_{1}^{\prime} \equiv 0(\bmod k)$.

Let us consider the coefficients for the 1 -chain $\beta^{\prime}$. For every face $f_{j}$ intersecting $f_{1}$, we have $a_{1}^{\prime}-a_{j}^{\prime} \equiv 0(\bmod k)$, which implies $a_{j}^{\prime} \equiv 0(\bmod k)$. Since $\partial \mathcal{P}$ is connected, repeating this argument we have $a_{i}^{\prime} \equiv 0(\bmod k)$ for all $i$. In addition, there are even $m_{i j}$ (the stabilizer of a vertex is a spherical triangle group), and hence $(9)$ also gives $a_{i}^{\prime}-b_{j}^{\prime} \equiv 0(\bmod k)$, which implies $b_{j}^{\prime} \equiv 0(\bmod k)$. Exactly the same argument as above gives then $b_{i}^{\prime} \equiv 0(\bmod k)$ for all $i$.

Since all coefficients of $\beta^{\prime}$ are divisible by $k$, we conclude that $\alpha=\partial_{2}\left(\beta^{\prime} / k\right) \in$ $\operatorname{im}\left(\partial_{2}\right)$, as desired.

We note that a similar method of proof can be used (in many cases) to show that $H_{0}$ is torsion-free. This approach is carried out in [10].

Corollary 2. Let $c f(\Gamma)$ be the number of conjugacy classes of elements of finite order in $\Gamma$, and $\chi(\mathcal{C})$ the Euler characteristic of the Bredon chain complex (6). Then we have

$$
H_{1} \cong \mathbb{Z}^{c f(\Gamma)-\chi(\mathcal{C})}
$$

Proof. The Euler characteristic of a chain complex coincides with the alternating sum of the ranks of the homology groups

$$
\chi(\mathcal{C})=\operatorname{rank}\left(H_{0}\right)-\operatorname{rank}\left(H_{1}\right)+\operatorname{rank}\left(H_{2}\right)-\operatorname{rank}\left(H_{3}\right) .
$$

Since $H_{3}=H_{2}=0$, we have $\operatorname{rank}\left(H_{1}\right)=\operatorname{rank}\left(H_{0}\right)-\chi(\mathcal{C})$, and $\operatorname{rank}\left(H_{0}\right)=$ cf $(\Gamma)$ [19]. Since $H_{1}$ is torsion-free (Theorem 2), the result follows.

Note that both $c f(\Gamma)$ and $\chi(\mathcal{C})$ can be obtained directly from the geometry of the polyhedron $\mathcal{P}$ or, equivalently, from the Coxeter integers $m_{i j}$. We show this explicitly in Section 5 .

Remark 1. Our results agree with a previous article by three of the authors [11], where we computed the rank of the Bredon homology for groups $\Gamma$ with a cocompact, 3-manifold model $X$ of the classifying space $\underline{E} \Gamma$. Firstly, note that Proposition 1 coincides with [11, Lemma 3], and, with respect to the vanishing of $H_{3}$, Proposition 2 follows from [11, Lemma 7]. The rank of $H_{2}(X)$ is given in [11, Corollary $14]$ by $\beta_{2}(Y)$ if $X / \Gamma$ is a closed oriented 3-manifold, or $s+t^{\prime}+2 t+\beta_{2}(Y)-1$ otherwise. Here $Y$ is the union of the closures of all interior faces of $X / \Gamma$ along with all the non-dihedral boundary components, $s$ is the number of orientable non-odd dihedral components, $t$ the number of orientable odd dihedral components (see [11] for definitions), $t^{\prime}$ is the number of orientable, odd, connected components in $\partial Y$, and $\beta_{2}()$ indicates the second Betti number. In our case, $X / G=\mathcal{P}$ has a unique boundary component, which must be dihedral (all edges stabilizers are of the form $D_{m_{i j}}$ with $m_{i j} \geq 2$ ), and there are no interior faces. Therefore $Y=\emptyset, t^{\prime}=0$ and either $s=0$ and $t=1$ (if all $m_{i j}$ are odd), or $s=0$ and $t=1$ (if there is at least one $m_{i j}$ even). Picking any vertex $v$ on $\partial P$, it has stabilizer which is a finite triangle group, and hence at least one $m_{i j}$ equals 2 . Thus we have indeed $s=0$ and $t=1$ and we conclude that $\operatorname{rank}\left(H_{2}\right)=0$, as expected. The rank of $H_{0}$ coincides with the number of conjugacy classes of elements of finite order in $\Gamma$. This number can be deduced from the 1-skeleton of a model of $\underline{E} \Gamma$, as explained in [11, §3.2], or in 
$\S 5.1$ below. Finally, the rank of $H_{1}$ is obtained, in both [11] and this article, from the rank of the other homology groups, and the Euler characteristic of the chain complex (5) (or (1) in [11]), which equals the alternating sum of the number of conjugacy classes in the stabilizers of $\Gamma$-orbits of cells.

To complete the computation of the Bredon homology, and hence of the equivariant $K$-homology, all that remains is the compute the torsion subgroup of $H_{0}$. We will show that in fact $H_{0}$ is also torsion-free.

Theorem 3. There is no torsion in $H_{0}$.

We postpone the proof to Section 4 below. An immediate consequence of Theorem 3 is

Corollary 3. $K_{0}^{\Gamma}(X)$ is torsion-free of rank $c f(\Gamma)$.

Combining Corollaries 1, 2, and 3 immediately yields the above Main Theorem. Moreover, in Section 5, we will give a formula for $c f(\Gamma)$ and $\chi(\mathcal{C})$ from the geometry of the polyhedron.

\section{NO TORSION IN $H_{0}$}

In this section, we give a proof of Theorem 3 inspired by the representation ring splitting technique of [22]. For this purpose, we establish a criterion for $\mathrm{H}_{0}^{\mathfrak{F i n}}\left(\Gamma ; R_{\mathbb{C}}\right)$ to be torsion-free which is quite efficient to check (by elementary linear algebra), and which is satisfied for $\Gamma$ a hyperbolic Coxeter group. The verification of that criterion relies on simultaneous base transformations of the representation rings, bringing the induction homomorphisms into the desired form, as carried out in Appendices A and B. An alternative, geometric, proof for Theorem 3 is outlined for hyperbolic Coxeter groups, and completed for a specific collection of hyperbolic Coxeter groups, in [10].

Definition 1. The vertex block of a given vertex $v$ in a Bredon chain complex differential matrix $\partial_{1}$ consists of all the blocks of $\partial_{1}$ that are representing maps induced (on complex representation rings from $\Gamma_{e} \rightarrow \Gamma_{v}$ ) by edges $e$ adjacent to $v$.

Therefore, denoting by $n_{0}$ the rank of $R_{\mathbb{C}}\left(\Gamma_{v}\right)$, if $v$ is adjacent to edges $e_{1}, e_{2}$ and $e_{3}$, with corresponding representation rings of rank $n_{1}, n_{2}$ and $n_{3}$, then its vertex block is a submatrix of $\partial_{1}$ (identified with its matrix representation after fixing a basis) of size $n_{1}+n_{2}+n_{3}$ times $n_{0}$.

Our proof of Theorem 3 consists of checking on the vertex blocks the following criterion that guarantees that $\mathrm{H}_{0}^{\mathfrak{F i n}}\left(\Gamma ; R_{\mathbb{C}}\right)$ is torsion-free.

Theorem 4. If there exists a base transformation such that all minors in all vertex blocks are in the set $\{-1,0,1\}$, then $\mathrm{H}_{0}^{\mathfrak{F i n}}\left(\Gamma ; R_{\mathbb{C}}\right)$ is torsion-free.

For the proof of this criterion, we will use a simple linear algebra lemma, which we prove again here for the sake of completeness, even though it was known to linear algebraists before the authors re-discovered it.

Lemma 2. For square matrices $M$ and $B$, we have

$$
\operatorname{det}\left(\begin{array}{cc}
M & 0 \\
* & B
\end{array}\right)=\operatorname{det}(M) \cdot \operatorname{det}(B) .
$$


Proof. Writing $M=\left(a_{i, j}\right)$ and denoting by $M^{i, j}$ the $(i, j)$-minor of $M$ (that is, the determinant of the block obtained by omitting the $i$-th row and the $j$-th column), we have

$$
\operatorname{det}\left(\begin{array}{cc}
M & 0 \\
* & B
\end{array}\right)=\sum_{j=1}^{\# M} a_{1, j}(-1)^{1+j} M^{1, j} \cdot \operatorname{det}(B),
$$

by iterating the development of the determinant by minors $(\# M-1)$ times, and making use of the zero block each time.

Proof of Theorem 4. As a generality on Smith Normal Forms, known already to Smith [24], we note that the elementary divisors of a matrix $A$ can be computed (up to multiplication by a unit) as $\alpha_{i}=\frac{d_{i}(A)}{d_{i-1}(A)}$, where $d_{i}(A)$ (called $i$-th determinant divisor) equals the greatest common divisor of all $i \times i$ minors of the matrix $A$, and $d_{0}(A):=1$.

Let us use the notation

$$
\operatorname{pre-rank}\left(\partial_{1}\right):=\operatorname{rank}_{\mathbb{Z}} C_{1}-\operatorname{rank}_{\mathbb{Z}} \operatorname{ker} \partial_{1},
$$

where $C_{1}$ is the module of 1-chains in the Bredon chain complex.

Then $\mathrm{H}_{0}^{\mathfrak{乛 i n}}\left(\Gamma ; R_{\mathbb{C}}\right)$ is torsion-free if and only if $\alpha_{i}= \pm 1$ for all $1 \leq i \leq \operatorname{pre-rank}\left(\partial_{1}\right)$, which, by the remark above, follows from finding an $(i \times i)$-minor in the Bredon chain complex differential matrix $\partial_{1}$ with value \pm 1 , for each $1 \leq i \leq \operatorname{pre-rank}\left(\partial_{1}\right)$. Let us show this is indeed the case, by induction on $i$.

Induction basis. For $i=1$, we observe that there are vertices with adjacent edges for the action of $\Gamma$, hence there are non-zero vertex blocks. As by assumption all the entries in the vertex blocks are in the set $\{-1,0,1\}$, there exists an entry of value \pm 1 .

Inductive step. Let $2 \leq i \leq \operatorname{pre-rank}\left(\partial_{1}\right)$, and assume that there exists an $(i-1) \times(i-1)$-minor of $\partial_{1}$ of value \pm 1 . We have to find an $i \times i$-minor of $\partial_{1}$ of value \pm 1 . Let $B^{\prime}$ be the $(i-1) \times(i-1)$-block of $\partial_{1}$, whose determinant is \pm 1 according to the inductive hypothesis. As $i \leq \operatorname{pre}-\operatorname{rank}\left(\partial_{1}\right)$, there exists a vertex block $V$ with the following property: After suitable base transformation, which puts $V$ into the upper left corner of $\partial_{1}$, the block $B^{\prime}$ can be extended to an $i \times i$-block

$$
B^{\prime \prime}=\operatorname{det}\left(\begin{array}{cc}
M & 0 \\
* & B
\end{array}\right)
$$

with $B$ a square sub-block of $B^{\prime}$, and $M$ a square sub-block of $V$. Here, $B$ is completely disjoint with $V$, and therefore we get the zero block in the upper right corner of $B^{\prime \prime}$ (note that the vertex blocks have been constructed to contain all entries from adjacent edges, so the remainders of their rows are zero). In case that $B^{\prime}$ is already disjoint with $V$, we simply have $B=B^{\prime}$, and $M$ is then a single entry of $V$. Otherwise, we construct $B$ as the maximal square sub-block of $B^{\prime}$ that has all of its rows and columns outside rows and columns in which $V$ is present. Then $M$ is constructed by taking the intersection of $B^{\prime}$ and $V$, and extending it to a square block of size $n-\operatorname{size}(B)$ inside $V$. Lemma 2 yields $\operatorname{det}\left(B^{\prime \prime}\right)=\operatorname{det}(M) \cdot \operatorname{det}(B)$. Now $\operatorname{det}(M) \in\{-1,0,1\}$ by the assumption that there is no torsion in the vertex blocks; and as $n \leq \operatorname{pre-rank}\left(\partial_{1}\right)$, we can reach $\operatorname{det}\left(B^{\prime \prime}\right) \neq 0$ and hence $\operatorname{det}(M) \neq 0$. For $B$ as a sub-block of $B^{\prime}, \operatorname{det}\left(B^{\prime}\right) \in\{-1,1\}$ entails $\operatorname{det}(B) \in\{-1,1\}$. This implies $\operatorname{det}\left(B^{\prime \prime}\right)= \pm 1$ and we are done. 
Using Theorem 4, the lack of non-trivial torsion in $H_{0}$ is a consequence of the following result, whose proof depends on the simultaneous base transformations in Appendix B.

Proposition 3. For a system of finite subgroups of types $A_{5} \times C_{2}, S_{4}, S_{4} \times C_{2}$, $\Delta(2,2,2)=\left(C_{2}\right)^{3}$ and $\Delta(2,2, m)=C_{2} \times D_{m}$ for $m \geq 3$ as vertex stabilizers, with their three 2-generator Coxeter subgroups as adjacent edge stabilizers, there is a simultaneous base transformation such that all vertex blocks have all their minors contained in the set $\{-1,0,1\}$.

Proof. We apply the base transformation specified in Appendix A. Then we already see that all of the induced maps (Appendix B, all Tables referenced in this proof can be found there) have all of their entries in the set $\{-1,0,1\}$. Next, we assemble the vertex blocks from the three vertex-edge-adjacency induced maps for any given vertex stabilizer type. By Tables 24 and 25, the vertex block of a stabilizer of type $D_{m}$ for $m \geq 3$ odd consists of

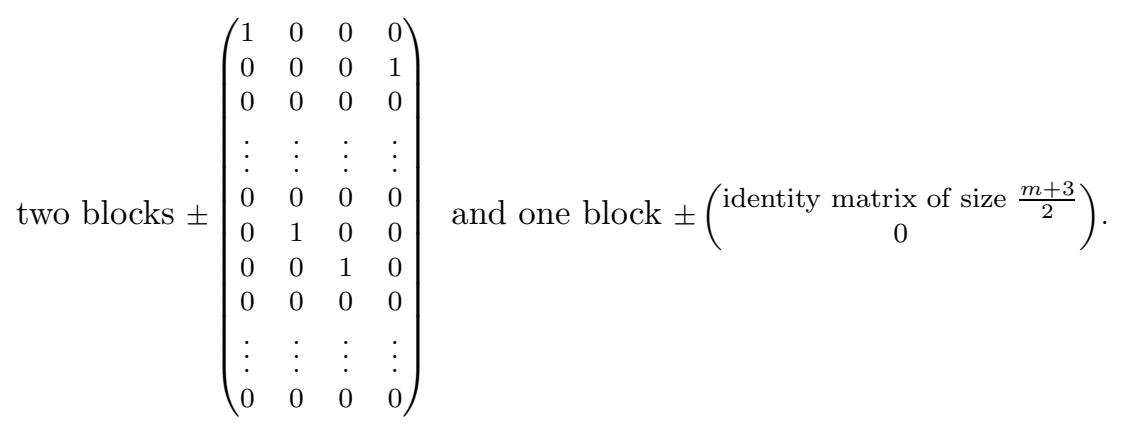

By Lemma 2, the columns which are concentrated in one entry \pm 1 cannot increase the absolute value of the determinant of a block which is being extended into them. We conclude that all minors are in $\{0, \pm 1\}$.

For $m \geq 6$ even, but not a power of 2 , Tables 26 and 27 yield the following vertex block, where each matrix block is specified up to orientation sign (we make this assumption from now on),

\begin{tabular}{|c|c|c|c|c|c|c|c|c|c|c|c|c|c|c|c|}
\hline$D_{m} \times C_{2}$ & \multicolumn{7}{|c|}{$D_{m} \hookrightarrow D_{m} \times C_{2}$} & \multicolumn{4}{|c|}{$D_{2} \hookrightarrow D_{m} \times C_{2}$} & \multicolumn{4}{|c|}{$D_{2} \hookrightarrow D_{m} \times C_{2}$} \\
\hline$\rho_{1} \otimes \chi_{1} \downarrow$ & 1 & 0 & 0 & 0 & 0 & 0 & 0 & 1 & 0 & 0 & 0 & 1 & 0 & 0 & 0 \\
\hline$\rho_{1} \otimes\left(\chi_{2}-\chi_{1}\right) \downarrow$ & 0 & 1 & 0 & 1 & 0 & 0 & 0 & 0 & 0 & 0 & 1 & 0 & 0 & 0 & 1 \\
\hline$\rho_{1} \otimes\left(\chi_{3}-\chi_{2}\right) \downarrow$ & 0 & 0 & 1 & -1 & 0 & 0 & 0 & 0 & 0 & 0 & 0 & 0 & 0 & 0 & 0 \\
\hline$\rho_{1} \otimes\left(\chi_{4}-\chi_{1}\right) \downarrow$ & 0 & 0 & 0 & 1 & 0 & 0 & 0 & 0 & 0 & 0 & 0 & 0 & 0 & 0 & 0 \\
\hline$\rho_{1} \otimes\left(\phi_{1}-\chi_{3}-\chi_{1}\right) \downarrow$ & 0 & 0 & 0 & 1 & 1 & 0 & 0 & 0 & 0 & 0 & 0 & 0 & 0 & 0 & 0 \\
\hline$\vdots \quad \rho_{1} \otimes\left(\phi_{p}-\phi_{p-1}\right) \downarrow \quad \vdots$ & 0 & 0 & 0 & 0 & 0 & $\ddots$ & 0 & : & : & : & $\vdots$ & $\vdots$ & $\vdots$ & $\vdots$ & $\vdots$ \\
\hline$\rho_{1} \otimes\left(\phi \frac{m}{2}-1-\phi \frac{m}{2}-2\right) \downarrow$ & 0 & 0 & 0 & 0 & 0 & 0 & 1 & 0 & 0 & 0 & 0 & 0 & 0 & 0 & 0 \\
\hline$\left(\rho_{2}-\rho_{1}\right) \otimes \chi_{1} \downarrow$ & & & & & & & & 0 & 1 & 0 & 0 & 0 & 1 & 0 & 0 \\
\hline$\left(\rho_{2}-\rho_{1}\right) \otimes\left(\chi_{2}-\chi_{1}\right) \downarrow$ & & & & & & & & 0 & 0 & 1 & 0 & 0 & 0 & 1 & 0 \\
\hline$\left(\rho_{2}-\rho_{1}\right) \otimes\left(\chi_{3}-\chi_{2}\right) \downarrow$ & & & & & & & & 0 & 0 & 0 & 0 & 0 & 0 & 0 & 0 \\
\hline$\left(\rho_{2}-\rho_{1}\right) \otimes\left(\chi_{4}+\chi_{3}-\chi_{2}-\chi_{1}\right) \downarrow$ & & & & $\mathbf{0}$ & & & & 0 & 0 & 0 & 0 & 0 & 0 & 0 & 0 \\
\hline$\left(\rho_{2}-\rho_{1}\right) \otimes\left(\phi_{1}-\chi_{2}-\chi_{1}\right) \downarrow$ & & & & & & & & 0 & 0 & 0 & 0 & 0 & 0 & 0 & 0 \\
\hline$: \quad \rho_{1} \otimes\left(\phi_{p}-\phi_{p-1}\right) \downarrow \quad:$ & & & & & & & & : & : & : & $\vdots$ & $\vdots$ & $\vdots$ & $\vdots$ & $\vdots$ \\
\hline$\rho_{1} \otimes\left(\phi \frac{m}{2}-1-\phi \frac{m}{2}-2\right) \downarrow$ & & & & & & & & 0 & 0 & 0 & 0 & 0 & 0 & 0 & 0 \\
\hline
\end{tabular}


By Lemma 2, we can ignore the rows and columns which have at most one entry \pm 1 , and reduce the above vertex block to the finite block

$$
\left(\begin{array}{cccccc}
1 & 0 & 1 & 0 & \pm 1 & \pm 1 \\
0 & 1 & -1 & 0 & 0 & 0 \\
0 & 0 & 1 & 0 & 0 & 0 \\
0 & 0 & 1 & 1 & 0 & 0
\end{array}\right)
$$

for which we can easily check that it has all its minors in $\{0, \pm 1\}$.

For $m \geq 4$ a power of 2 , Tables 28 and 29 yield the following vertex block,

\begin{tabular}{|c|c|c|c|c|c|c|c|c|c|c|c|c|c|c|c|}
\hline$D_{m} \times C_{2}$ & \multicolumn{7}{|c|}{$D_{m} \hookrightarrow D_{m} \times C_{2}$} & \multicolumn{4}{|c|}{$D_{2} \hookrightarrow D_{m} \times C_{2}$} & \multicolumn{4}{|c|}{$D_{2} \hookrightarrow D_{m} \times C_{2}$} \\
\hline$\rho_{1} \otimes \chi_{1} \downarrow$ & 1 & 0 & 0 & 0 & 0 & 0 & 0 & 1 & 0 & 0 & 0 & 1 & 0 & 0 & 0 \\
\hline$\rho_{1} \otimes\left(\chi_{2}-\chi_{1}\right) \downarrow$ & 0 & 1 & 0 & 1 & 0 & 0 & 0 & 0 & 0 & 0 & 1 & 0 & 0 & 0 & 1 \\
\hline$\rho_{1} \otimes\left(\chi_{3}-\chi_{1}\right) \downarrow$ & 0 & 1 & 1 & 0 & 0 & 0 & 0 & 0 & 0 & 0 & 0 & 0 & 0 & 0 & 0 \\
\hline$\rho_{1} \otimes\left(\chi_{4}-\chi_{2}\right) \downarrow$ & 0 & -1 & 0 & 0 & 0 & 0 & 0 & 0 & 0 & 0 & 0 & 0 & 0 & 0 & 0 \\
\hline$\rho_{1} \otimes\left(\phi_{1}-\chi_{2}-\chi_{1}\right) \downarrow$ & 0 & 0 & 1 & 0 & 1 & 0 & 0 & 0 & 0 & 0 & 0 & 0 & 0 & 0 & 0 \\
\hline$\vdots \quad \rho_{1} \otimes\left(\phi_{p}-\phi_{p-1}\right) \downarrow \quad \vdots$ & 0 & 0 & 0 & 0 & 0 & & 0 & : & $:$ & : & : & $\vdots$ & $\vdots$ & $\vdots$ & $\vdots$ \\
\hline$\left(\phi \frac{m}{2}-1-\phi \frac{m}{2}-2\right) \downarrow$ & 0 & 0 & 0 & 0 & 0 & 0 & 1 & 0 & 0 & 0 & 0 & 0 & 0 & 0 & 0 \\
\hline$\left(\rho_{2}-\rho_{1}\right) \otimes \chi_{1} \downarrow$ & & & & & & & & 0 & 1 & 0 & 0 & 0 & 1 & 0 & 0 \\
\hline$\left(\rho_{2}-\rho_{1}\right) \otimes\left(\chi_{2}-\chi_{1}\right) \downarrow$ & & & & & & & & 0 & 0 & 1 & 0 & 0 & 0 & 1 & 0 \\
\hline$\left(\rho_{2}-\rho_{1}\right) \otimes\left(\chi_{3}-\chi_{1}\right) \downarrow$ & & & & & & & & 0 & 0 & 0 & 0 & 0 & 0 & 0 & 0 \\
\hline$\left(\rho_{2}-\rho_{1}\right) \otimes\left(\chi_{4}+\chi_{3}-\chi_{2}-\chi_{1}\right) \downarrow$ & & & & $\mathbf{0}$ & & & & 0 & 0 & 0 & 0 & 0 & 0 & 0 & 0 \\
\hline$\left(\rho_{2}-\rho_{1}\right) \otimes\left(\phi_{1}-\chi_{2}-\chi_{1}\right) \downarrow$ & & & & & & & & 0 & 0 & 0 & 0 & 0 & 0 & 0 & 0 \\
\hline$\vdots \quad \rho_{1} \otimes\left(\phi_{p}-\phi_{p-1}\right) \downarrow \quad \vdots$ & & & & & & & & : & : & : & : & : & : & : & $:$ \\
\hline$\rho_{1} \otimes\left(\phi \frac{m}{2}-1-\phi \frac{m}{2}-2\right) \downarrow$ & & & & & & & & 0 & 0 & 0 & 0 & 0 & 0 & 0 & 0 \\
\hline
\end{tabular}

By Lemma 2, we can ignore the rows and columns which have at most one entry \pm 1 , and reduce the above vertex block to the finite block

$$
\left(\begin{array}{ccccccccc}
1 & 0 & 0 & 0 & 0 & \pm 1 & 0 & \pm 1 & 0 \\
0 & 1 & 0 & 1 & 0 & 0 & \pm 1 & 0 & \pm 1 \\
0 & 1 & 1 & 0 & 0 & 0 & 0 & 0 & 0 \\
0 & -1 & 0 & 0 & 0 & 0 & 0 & 0 & 0 \\
0 & 0 & 1 & 0 & 1 & 0 & 0 & 0 & 0
\end{array}\right)
$$

for which we can easily check that it has all its minors in $\{0, \pm 1\}$. For the finitely many remaining stabilizer types, we can proceed case-by-case: we input each vertex block into a computer routine which computes all of its minors (such a routine is straightforward to implement and takes approximately two seconds per vertex block on a standard computer; the third author's implementation is available at http://math.uni.lu/ rahm/vertexBlocks/). Note that for the groups under consideration, the matrix rank of the vertex block is at most 7 , so the $8 \times 8$-minors are all zero, and it is enough to compute the $n \times n$-minors for $n \leq 7$.

Corollary 4. For any Coxeter group $\Gamma$ having a system of finite subgroups of types $\Delta(2,2,2)=\left(C_{2}\right)^{3}, \Delta(2,2, m)=C_{2} \times D_{m}$ for $m \geq 3, S_{4}, S_{4} \times C_{2}$ or $A_{5} \times C_{2}$ as vertex stabilizers, we have that the Bredon homology group $\mathrm{H}_{0}^{\mathfrak{F i n}}\left(\Gamma ; R_{\mathbb{C}}\right)$ is torsionfree.

Remark 2. Note that to use the criterion in Theorem 4 for proving that $\mathrm{H}_{n}^{\mathfrak{F i n}}\left(\Gamma ; R_{\mathbb{C}}\right)$ is torsion-free for a group $\Gamma$, the following should be taken into account:

(a) The proof of the theorem, stated for $n=0$, implicitly uses the fact that $H_{0}(\underline{B} \Gamma ; \mathbb{Z})$ is always torsion-free. To extend the theorem to $n>0$, one should either work with groups for which $H_{n}(\underline{\mathrm{B}} \Gamma ; \mathbb{Z})$ is torsion-free (such as Coxeter groups), or use the splitting described in section 7 of [22] in order to make a statement only about the part of $\mathrm{H}_{n}^{\mathfrak{乛 i n}}\left(\Gamma ; R_{\mathbb{C}}\right)$ complementary to $H_{n}(\underline{\mathrm{B}} \Gamma ; \mathbb{Z})$. 
(b) Before trying to prove the hypothesis in the theorem using base transformations (as in Appendix A), which can become quite laborious, one should construct the vertex blocks without any base transformation and compute their elementary divisors. If there exists a suitable simultaneous base transformation which proves the criterion, then those elementary divisors must be in the set $\{-1,0,1\}$.

\section{5. $c f(\Gamma)$ AND $\chi(\mathcal{C})$ FROM THE GEOMETRY OF $\mathcal{P}$}

Let $\Gamma$ be the reflection group of the compact 3-dimensional hyperbolic polyhedron $\mathcal{P}$. In this section, we compute the number of conjugacy classes of elements of finite order of $\Gamma, c f(\Gamma)$, and the Euler characteristic of the Bredon chain complex (5), $\chi(\mathcal{C})$, from the geometry of the polyhedron $\mathcal{P}$. This makes our Bredon homology and equivariant $K$-theory results, in particular the above Main Theorem, explicit.

5.1. Conjugacy classes of elements of finite order. We now give an algorithm to calculate $c f(\Gamma)$, the number of conjugacy classes of elements of finite order in the Coxeter group $\Gamma$. We know that each element of finite order can be conjugated to one which stabilizes one of the $k$-dimensional faces of the polyhedron, for some $k \in\{0,1,2\}$. Of course, the only element which stabilizes all faces is the identity element. Let us set that aside, and consider the non-identity elements, to which we associate the integer $k$. We now count the elements according to the integer $k$, in descending order.

Case $k=2$ : These are the conjugacy classes represented by the canonical generators of the Coxeter group $\Gamma$. The number of these is given by the total number $\left|\mathcal{P}^{(2)}\right|$ of facets of the polyhedron $\mathcal{P}$.

Case $k=1$ : These elements are edge stabilizers which are not conjugate to the stabilizer of a face. We first note that there are some possible conjugacies between edge stabilizers. Geometrically, these occur when there is a geodesic $\gamma \subset \mathbb{H}^{3}$ whose projection onto the fundamental domain $\mathcal{P}$ covers multiple edges inside the 1-skeleton $\mathcal{P}^{(1)}$. A detailed analysis of when this can happen is given in [9]. Following the description in that paper, we decompose the 1-skeleton into equivalence classes of edges, where two edges are equivalent if there exists a geodesic whose projection passes through both edges. Denote by $\left[\mathcal{P}^{(1)}\right]$ the set of equivalence classes of edges, and note that each equivalence class $[e]$ has a well defined group associated to it, which is just the dihedral group $\Gamma_{e}$ stabilizing a representative edge. We can thus count the conjugacy classes in the corresponding dihedral group, and discard the three conjugacy classes already accounted for (the conjugacy class of the two canonical generators counted in case $k=2$, as well as the identity). Thus the contribution from finite elements of this type is given by

$$
\sum_{[e] \in\left[\mathcal{P}^{(1)}\right]}\left(c\left(\Gamma_{e}\right)-3\right) .
$$

(Recall that $c\left(D_{m}\right)$, the number of conjugacy classes in a dihedral group of order $2 m$, is $m / 2+3$ if $m$ even, and $(m-1) / 2+2$ if $m$ is odd.)

Case $k=0$ : Finally, we consider the contribution from the elements in the vertex stabilizers which have not already been counted. That is to say, for each vertex $v \in$ $\mathcal{P}^{(0)}$, we count the conjugacy classes of elements in the corresponding 3-generated spherical triangle group, which cannot be conjugated into one of the canonical 2generated special subgroups. This number, $\bar{c}\left(\Gamma_{v}\right)$, depends only on the isomorphism 
type of the spherical triangle group $\Gamma_{v}$, see Table 1 . The contribution from these types of finite elements is thus

$$
\sum_{v \in \mathcal{P}^{(0)}} \bar{c}\left(\Gamma_{v}\right)
$$

\begin{tabular}{c|cc}
$\Gamma_{v}$ & $c\left(\Gamma_{v}\right)$ & $\bar{c}\left(\Gamma_{v}\right)$ \\
\hline$\Delta(2,2, m)$ & $2 c\left(D_{m}\right)$ & $c\left(D_{m}\right)-3$ \\
$\Delta(2,3,3)$ & 5 & 1 \\
$\Delta(2,3,4)$ & 10 & 3 \\
$\Delta(2,3,5)$ & 10 & 5
\end{tabular}

TABLE 1. Number of conjugacy classes in spherical triangle groups. The left column is the total number (cf. Appendix A), and the right column the number of those not conjugated into one of the three canonical 2-generated special subgroups.

Combining all these, we obtain the desired (combinatorial) formula for the number of conjugacy classes of elements of finite order inside the group $\Gamma$ :

$$
c f(\Gamma)=1+\left|\mathcal{P}^{(2)}\right|+\sum_{[e] \in\left[\mathcal{P}^{(1)}\right]}\left(c\left(\Gamma_{e}\right)-3\right)+\sum_{v \in \mathcal{P}^{(0)}} \bar{c}\left(\Gamma_{v}\right) .
$$

5.2. Euler characteristic. The Euler characteristic of the Bredon chain complex can be easily calculated from the stabilizers of the various faces of the polyhedron $\mathcal{P}$, according to the formula:

$$
\chi(\mathcal{C})=\sum_{f \in \mathcal{P}}(-1)^{\operatorname{dim}(f)} \operatorname{dim}\left(R_{\mathbb{C}}\left(\Gamma_{f}\right)\right) .
$$

Depending on the dimension of the faces, we know exactly what the dimension of the complex representation ring is (the number of conjugacy classes in the stabilizer):

- for the 3-dimensional face (the interior), the stabilizer is trivial, so there is a 1-dimensional complex representation ring;

- for the 2-dimensional faces, the stabilizer are $\mathbb{Z}_{2}$, and there is a 2-dimensional complex representation ring;

- for the 1-dimensional faces $e$, the stabilizers are dihedral groups, and there is a $c\left(\Gamma_{e}\right)$-dimensional complex representation ring;

- for the 0-dimensional faces $v$, the stabilizers are spherical triangle groups, and there is a $c\left(\Gamma_{v}\right)$-dimensional complex representation ring.

Putting these together, we obtain

$$
\chi(\mathcal{C})=-1+2\left|\mathcal{P}^{(2)}\right|-\sum_{e \in \mathcal{P}^{(1)}} c\left(\Gamma_{e}\right)+\sum_{v \in \mathcal{P}^{(0)}} c\left(\Gamma_{v}\right) .
$$

All in all, we have a more explicit version of the above Main Theorem, from the geometry of the polyhedron $\mathcal{P}$. 
Main Theorem (explicit). Let $\Gamma$ be a cocompact 3-dimensional hyperbolic reflection group, generated by reflections in the side of a hyperbolic polyhedron $\mathcal{P} \subset \mathbb{H}^{3}$. Then $K_{0}\left(C_{r}^{*}(\Gamma)\right)$ is a torsion-free abelian group of rank

$$
c f(\Gamma)=1+\left|\mathcal{P}^{(2)}\right|+\sum_{[e] \in\left[\mathcal{P}^{(1)}\right]}\left(c\left(\Gamma_{e}\right)-3\right)+\sum_{v \in \mathcal{P}^{(0)}} \bar{c}\left(\Gamma_{v}\right),
$$

and $K_{1}\left(C_{r}^{*}(\Gamma)\right)$ is a torsion-free abelian group of rank

$c f(\Gamma)-\chi(\mathcal{C})=2-\left|\mathcal{P}^{(2)}\right|+\sum_{[e] \in\left[\mathcal{P}^{(1)}\right]}\left(c\left(\Gamma_{e}\right)-3\right)+\sum_{e \in \mathcal{P}^{(1)}} c\left(\Gamma_{e}\right)-\sum_{v \in \mathcal{P}^{(0)}}\left(c\left(\Gamma_{v}\right)-\bar{c}\left(\Gamma_{v}\right)\right)$, and the values $c\left(\Gamma_{v}\right)$ and $\bar{c}\left(\Gamma_{v}\right)$ can be obtained from Table 1 .

\section{REFERENCES}

[1] A. Adem. Characters and K-theory of discrete groups. Invent. Math., 114(3):489-514, 1993.

[2] E. M. Andreev. Convex polyhedra of finite volume in Lobačevskiǔ space. Mat. Sb. (N.S.), 83 (125):256-260, 1970

[3] M. Bożejko, T. Januszkiewicz, and R. J. Spatzier. Infinite Coxeter groups do not have Kazhdan's property. J. Operator Theory, 19(1):63-67, 1988.

[4] J. F. Davis and W. Lück. The topological K-theory of certain crystallographic groups. J. Noncommut. Geom., 7(2):373-431, 2013.

[5] R. Flores, S. Pooya, and A. Valette. K-homology and K-theory for the lamplighter groups of finite groups. To appear in Proc. LMS, arXiv:1610.02798, 2016.

[6] N. Higson and G. Kasparov. Operator $K$-theory for groups which act properly and isometrically on Hilbert space. Electron. Res. Announc. Amer. Math. Soc., 3:131-142 (electronic), 1997.

[7] O. Isely. K-theory and K-homology for semi-direct products of $\mathbb{Z}_{2}$ by $\mathbb{Z}$. 2011. Thèse de doctorat : Université de Neuchâtel (Switzerland).

[8] J.-F. Lafont, B. A. Magurn, and I. J. Ortiz. Lower algebraic $K$-theory of certain reflection groups. Math. Proc. Cambridge Philos. Soc., 148(2):193-226, 2010.

[9] J.-F. Lafont and I. J. Ortiz. Lower algebraic $K$-theory of hyperbolic 3-simplex reflection groups. Comment. Math. Helv., 84(2):297-337, 2009.

[10] J.-F. Lafont, I. J. Ortiz, A. D. Rahm and R. J. Sánchez-García. Equivariant K-homology for hyperbolic reflection groups. Preprint version of the present paper, arXiv:1707.05133, 2017.

[11] J.-F. Lafont, I. J. Ortiz, and R. J. Sánchez-García. Rational equivariant $K$-homology of low dimensional groups. In Topics in noncommutative geometry, volume 16 of Clay Math. Proc., pages 131-163. Amer. Math. Soc., Providence, RI, 2012.

[12] M. Langer and W. Lück. Topological $K$-theory of the group $C^{*}$-algebra of a semi-direct product $\mathbb{Z}^{n} \rtimes \mathbb{Z} / m$ for a free conjugation action. J. Topol. Anal., 4(2):121-172, 2012.

[13] W. Lück. Chern characters for proper equivariant homology theories and applications to $K$ and $L$-theory. J. Reine Angew. Math., 543:193-234, 2002.

[14] W. Lück. $K$ - and $L$-theory of the semi-direct product of the discrete 3-dimensional Heisenberg group by $\mathbb{Z} / 4$. Geom. Topol., 9:1639-1676, 2005.

[15] W. Lück. Rational computations of the topological $K$-theory of classifying spaces of discrete groups. J. Reine Angew. Math., 611:163-187, 2007.

[16] W. Lück and B. Oliver. Chern characters for the equivariant $K$-theory of proper $G$-CWcomplexes. In Cohomological methods in homotopy theory (Bellaterra, 1998), volume 196 of Progr. Math., pages 217-247. Birkhäuser, Basel, 2001.

[17] W. Lück and H. Reich. The Baum-Connes and the Farrell-Jones conjectures in $K$ - and $L$ theory. In Handbook of K-theory. Vol. 1, 2, pages 703-842. Springer, Berlin, 2005.

[18] W. Lück and R. Stamm. Computations of $K$ - and $L$-theory of cocompact planar groups. K-Theory, 21(3):249-292, 2000.

[19] G. Mislin. Equivariant $K$-homology of the classifying space for proper actions. In Proper group actions and the Baum-Connes conjecture, Adv. Courses Math. CRM Barcelona, pages 1-78. Birkhäuser, Basel, 2003.

[20] G. Mislin and A. Valette. Proper group actions and the Baum-Connes conjecture. Advanced Courses in Mathematics. CRM Barcelona. Birkhäuser Verlag, Basel, 2003. 
[21] S. Pooya and A. Valette. $K$-theory for the $C^{*}$-algebras of the solvable Baumslag-Solitar groups. Preprint, arXiv:1610.02798, 2016.

[22] A. D. Rahm. Sur la $K$-homologie équivariante de $\mathrm{PSL}_{2}$ sur les entiers quadratiques imaginaires. Ann. Inst. Fourier, 66(4):1667-1689, 2016.

[23] J.-P. Serre. Linear representations of finite groups. Springer-Verlag, New York-Heidelberg, 1977. Translated from the second French edition by Leonard L. Scott, Graduate Texts in Mathematics, Vol. 42.

[24] H. J. S. Smith. On systems of linear indeterminate equations and congruences. Philosophical transactions of the Royal Society of London, 151:293-326, 1861.

[25] M. Yang. Crossed products by finite groups acting on low dimensional complexes and applications. ProQuest LLC, Ann Arbor, MI, 1997. Thesis (Ph.D.)-The University of Saskatchewan (Canada).

\section{Appendix A. Character tables And Base transformations}

In this Appendix, we list the character tables of all the groups involved in the Bredon chain complex (6), that is, finite Coxeter subgroups of $\Gamma$ up to rank three. This serves as a reference for the main text, and fixes the notation. In the character tables below, rows correspond to irreducible representations, and columns to representatives of conjugacy classes, written in term of the Coxeter generators $s_{1}, \ldots, s_{n}$ in a fixed Coxeter presentation of $\Gamma$, as in (1).

In addition, for each character table, we apply elementary row operations to obtain the transformed tables needed for Appendix B, which are in turn used in our proof that $H_{0}$ is torsion-free (Section 4). Although the rows of the transformed tables do no more consist of irreducible characters, it is easy to check that they still constitute bases of the complex representation rings.

Note that, for consistency across subgroups, we will pay attention to the order of the Coxeter generators in a subgroup. We write $e$ for the identity element in $\Gamma$.

A.1. Rank 0. This is the trivial group, with character table given below.

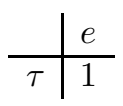

TABlE 2. Character table of the trivial group.

A.2. Rank 1. A rank 1 Coxeter group is a cyclic group of order 2. Write $s_{i}$ for its Coxeter generator, then its character table is Table 3 below.

\begin{tabular}{r|rr}
$C_{2}$ & $e$ & $s_{i}$ \\
\hline$\rho_{1}$ & 1 & 1 \\
$\rho_{2}$ & 1 & -1
\end{tabular}

TABLE 3. Character table of $\left\langle s_{i}\right\rangle \cong C_{2}$. 
A.3. Rank 2. A finite rank 2 Coxeter group with Coxeter generators $s_{i}$ and $s_{j}$ is a dihedral group of order $m=m_{i j} \geq 2$,

$$
D_{m}=\left\langle s_{i}, s_{j} \mid s_{i}^{2}=s_{j}^{2}=\left(s_{i} s_{j}\right)^{m}\right\rangle .
$$

The character table of this group is given in Table 4 below, where $0 \leq r \leq m-1$, $p$ varies between 1 and $m / 2-1$ if $m$ is even or $(m-1) / 2$ if $m$ is odd, and the hat denotes a character which appear only when $m$ is even. In order to be consistent,

\begin{tabular}{c|cc}
$D_{m}$ & $\left(s_{i} s_{j}\right)^{r}$ & $s_{j}\left(s_{i} s_{j}\right)^{r}$ \\
\hline$\chi_{1}$ & 1 & 1 \\
$\chi_{2}$ & 1 & -1 \\
$\widehat{\chi_{3}}$ & $(-1)^{r}$ & $(-1)^{r}$ \\
$\widehat{\chi_{4}}$ & $(-1)^{r}$ & $(-1)^{r+1}$ \\
$\phi_{p}$ & $2 \cos \left(\frac{2 \pi p r}{m}\right)$ & 0
\end{tabular}

TABLE 4. Character table of $\left\langle s_{i}, s_{j}>\cong D_{m}\right.$, where $i<j$.

we assume the Coxeter generators are ordered so that $i<j$. If $j<i$, then the character table is identical except that the third and fourth rows (the characters $\widehat{\chi}_{3}$ and $\left.\widehat{\chi_{4}}\right)$ are interchanged, since $\left(s_{j} s_{i}\right)^{r}=\left(s_{i} s_{j}\right)^{-r}$ and $s_{i}\left(s_{j} s_{i}\right)^{r}=s_{j}\left(s_{i} s_{j}\right)^{-r-1}$.

For the case $m=2$, that is, $D_{2}=C_{2} \times C_{2}$, we will sometimes use the notation coming from the character table of $C_{2}$ (Table 3 ) instead. (Recall that the irreducible characters of a direct product $G \times H$ are obtained from the irreducible characters of $G$ and $H$ as $\rho_{i} \otimes \tau_{j}$, where $\left(\rho_{i} \otimes \tau_{j}\right)(g, h)=\rho_{i}(g) \cdot \tau_{j}(h)$.) This gives the notation and characters in Table 5 , which are equivalent to Table 4 with $\rho_{1} \otimes \rho_{1}=\chi_{1}$, $\rho_{1} \otimes \rho_{2}=\chi_{4}, \rho_{2} \otimes \rho_{1}=\chi_{3}$ and $\rho_{2} \otimes \rho_{2}=\chi_{2}$. As before, we assume $i<j$, or, if $j<i$, the third and fourth rows (characters) must be interchanged.

\begin{tabular}{c|rrrr}
$C_{2} \times C_{2}$ & $e$ & $s_{i}$ & $s_{j}$ & $s_{i} s_{j}$ \\
\hline$\rho_{1} \otimes \rho_{1}$ & 1 & 1 & 1 & 1 \\
$\rho_{1} \otimes \rho_{2}$ & 1 & 1 & -1 & -1 \\
$\rho_{2} \otimes \rho_{1}$ & 1 & -1 & 1 & -1 \\
$\rho_{2} \otimes \rho_{2}$ & 1 & -1 & -1 & 1
\end{tabular}

TABLE 5. Alternative character table of $\left\langle s_{i}, s_{j}\right\rangle \cong D_{2}=C_{2} \times C_{2}$, $i<j$.

We now give the base transformations of the character table of $D_{m}$ needed later, shown in Tables 6,7 and 8.

A.4. Rank 3. A finite rank 3 Coxeter subgroup is one of the spherical triangle groups $\Delta(2,2, m)$, with $m \geq 2, \Delta(2,3,3), \Delta(2,3,4)$, or $\Delta(2,3,5)$, using the notation in (2), or, more compactly, the Coxeter diagrams in Figure 1.

In these diagrams, the vertices represent Coxeter generators and the edges are labelled by $m_{i j}$, with the conventions: no edge if $m_{i j}=2$, and no label if $m_{i j}=3$. 


\begin{tabular}{c|cccc}
$D_{2}$ & $e$ & $s_{i} s_{j}$ & $s_{i}$ & $s_{j}$ \\
\hline$\sum \chi_{i}$ & 4 & 0 & 0 & 0 \\
$\chi_{2}+\chi_{3}$ & 2 & 0 & -2 & 0 \\
$\chi_{3}$ & 1 & -1 & -1 & 1 \\
$\chi_{3}+\chi_{4}$ & 2 & -2 & 0 & 0
\end{tabular}

TABLE 6 . Base transformation of the character table of $D_{2}$.

\begin{tabular}{c|ccccc}
$D_{m}$ & $e$ & $s_{i}$ & $s_{i} s_{j}$ & $\left(s_{i} s_{j}\right)^{r}$ & $\left(s_{i} s_{j}\right)^{\frac{m-1}{2}}$ \\
\hline$\chi_{1}+\chi_{2}+2 \sum_{p=1}^{\frac{m-1}{2}} \phi_{p}$ & $2 m$ & 0 & 0 & $\ldots$ & 0 \\
$\chi_{2}+\sum_{p=1}^{\frac{m-1}{2}} \phi_{p}$ & $m$ & -1 & 0 & $\ldots$ & 0 \\
$\sum_{p=1}^{\frac{m-1}{2}} \phi_{p}$ & $m-1$ & 0 & -1 & $\ldots$ & -1 \\
$\vdots \quad \sum_{p=k}^{\frac{m-1}{2}} \phi_{p}$ & $m-2 k+1$ & $\vdots$ & $a_{k, 1}$ & $a_{k, r}$ & $a_{k, \frac{m-1}{2}}$ \\
$\phi_{\frac{m-1}{2}}$ & 2 & 0 & $b_{1}$ & $b_{r}$ & $b_{\frac{m-1}{2}}$
\end{tabular}

TABLE 7. Base transformation of the character table of $D_{m}, m \geq 3$ odd. Here, $a_{k, r}:=\sum_{p=k}^{\frac{m-1}{2}} 2 \cos \left(\frac{2 \pi p r}{m}\right), b_{r}:=2 \cos \left(\frac{\pi(m-1) r}{m}\right)$, and $1<k, r<\frac{m-1}{2}$.

\begin{tabular}{c|cccccc}
$D_{m}$ & $e$ & $s_{i}$ & $s_{i} s_{j}$ & $\left(s_{i} s_{j}\right)^{r}$ & $\left(s_{i} s_{j}\right)^{\frac{m}{2}}$ & $s_{j} s_{i} s_{j}$ \\
\hline$\sum_{p=1}^{4} \chi_{p}+2 \sum_{p=1}^{\frac{m l}{2}-1} \phi_{p}$ & $2 m$ & 0 & 0 & $\ldots$ & 0 & 0 \\
$\chi_{2}+\chi_{3}+\sum_{p=1}^{\frac{m}{2}-1} \phi_{p}$ & $m$ & 0 & 0 & $\ldots$ & 0 & -2 \\
$\chi_{3}+\sum_{p=1}^{\frac{m}{2}}-1 \phi_{p}$ & $m-1$ & 1 & -1 & $\ldots$ & -1 & -1 \\
$\chi_{2}+\chi_{4}+\sum_{p=1}^{\frac{m}{2}}-1$ & $m$ & -2 & 0 & $\ldots$ & 0 & 0 \\
$\sum_{p=1}^{\frac{m}{2}}-1 \phi_{p}$ & $m-2$ & 0 & 0 & $-1-(-1)^{r}$ & $-1-(-1)^{\frac{m}{2}}$ & 0 \\
$\vdots \quad \sum_{p=k}^{\frac{m}{2}-1} \phi_{p} \vdots$ & $m-2 k$ & $\vdots$ & $a_{k, 1}$ & $a_{k, r}$ & $2 \sum_{p=k}^{\frac{m}{2}-1}(-1)^{p}$ & 0 \\
$\phi_{\frac{m}{2}-1}$ & 2 & 0 & $b_{1}$ & $b_{r}$ & $2(-1)^{\frac{m}{2}-1}$ & 0
\end{tabular}

TABLE 8. Base transformation of the character table of $D_{m}, m \geq$ 4 even. Here, $a_{k, r}:=\sum_{p=k}^{\frac{m}{2}-1} 2 \cos \left(\frac{2 \pi p r}{m}\right), b_{r}:=2 \cos \left(\frac{\pi(m-2) r}{m}\right)$, $1<k<\frac{m}{2}-1$ and $1<r<\frac{m}{2}$.
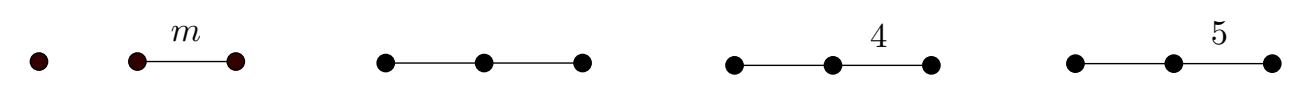

Figure 1. Coxeter diagrams of rank 3 spherical Coxeter groups.

A.4.1. $\Delta(2,2,2)$. This triangle group is isomorphic to $C_{2} \times C_{2} \times C_{2}$ and we have irreducible characters $\rho_{a b c}:=\rho_{a} \otimes \rho_{b} \otimes \rho_{c}, a, b, c \in\{1,2\}$, from Table 3, listed in Table 9 below, where $\rho_{a} \otimes \rho_{b} \otimes \rho_{c}(x)=\rho_{a}\left(x_{1}\right) \cdot \rho_{b}\left(x_{2}\right) \cdot \rho_{c}\left(x_{3}\right)$ for all $x=$ 
$\left(x_{1}, x_{2}, x_{3}\right) \in C_{2} \times C_{2} \times C_{2}$. Here we assume that we have ordered the Coxeter

\begin{tabular}{c|rrrrrrrr}
$C_{2} \times C_{2} \times C_{2}$ & $e$ & $s_{i}$ & $s_{j}$ & $s_{k}$ & $s_{i} s_{j}$ & $s_{i} s_{k}$ & $s_{j} s_{k}$ & $s_{i} s_{j} s_{k}$ \\
\hline$\rho_{111}:=\rho_{1} \otimes \rho_{1} \otimes \rho_{1}$ & 1 & 1 & 1 & 1 & 1 & 1 & 1 & 1 \\
$\rho_{112}:=\rho_{1} \otimes \rho_{1} \otimes \rho_{2}$ & 1 & 1 & 1 & -1 & 1 & -1 & -1 & -1 \\
$\rho_{121}:=\rho_{1} \otimes \rho_{2} \otimes \rho_{1}$ & 1 & 1 & -1 & 1 & -1 & 1 & -1 & -1 \\
$\rho_{122}:=\rho_{1} \otimes \rho_{2} \otimes \rho_{2}$ & 1 & 1 & -1 & -1 & -1 & -1 & 1 & 1 \\
$\rho_{211}:=\rho_{2} \otimes \rho_{1} \otimes \rho_{1}$ & 1 & -1 & 1 & 1 & -1 & -1 & 1 & -1 \\
$\rho_{212}:=\rho_{2} \otimes \rho_{1} \otimes \rho_{2}$ & 1 & -1 & 1 & -1 & -1 & 1 & -1 & 1 \\
$\rho_{221}:=\rho_{2} \otimes \rho_{2} \otimes \rho_{1}$ & 1 & -1 & -1 & 1 & 1 & -1 & -1 & 1 \\
$\rho_{222}:=\rho_{2} \otimes \rho_{2} \otimes \rho_{2}$ & 1 & -1 & -1 & -1 & 1 & 1 & 1 & -1
\end{tabular}

TABLE 9. Character table of $\left\langle s_{i}, s_{j}, s_{k}\right\rangle \cong \Delta(2,2,2)=C_{2} \times C_{2} \times$ $C_{2}, i<j<k$, from Table 3 .

generators $s_{i}, s_{j}, s_{k}$ so that $i<j<k$. Finally, the base transformation of the character table of $\Delta(2,2,2)$ needed later is shown in Table 10.

\begin{tabular}{c|rrrrrrrr}
$C_{2} \times C_{2} \times C_{2}$ & $e$ & $s_{i}$ & $s_{j}$ & $s_{k}$ & $s_{i} s_{j}$ & $s_{i} s_{k}$ & $s_{j} s_{k}$ & $s_{i} s_{j} s_{k}$ \\
\hline$\rho_{111}$ & 1 & 1 & 1 & 1 & 1 & 1 & 1 & 1 \\
$\rho_{112}-\rho_{111}$ & 0 & 0 & 0 & -2 & 0 & -2 & -2 & -2 \\
$\rho_{121}-\rho_{111}$ & 0 & 0 & -2 & 0 & -2 & 0 & -2 & -2 \\
$\rho_{122}-\rho_{121}$ & 0 & 0 & 0 & -2 & 0 & -2 & 2 & 2 \\
$\rho_{211}-\rho_{111}$ & 0 & -2 & 0 & 0 & -2 & -2 & 0 & -2 \\
$\rho_{212}-\rho_{211}$ & 0 & 0 & 0 & -2 & 0 & 2 & -2 & 2 \\
$\rho_{221}-\rho_{121}$ & 0 & -2 & 0 & 0 & 2 & -2 & 0 & 2 \\
$\rho_{222}-\rho_{221}$ & 0 & 0 & 0 & -2 & 0 & 2 & 2 & -2
\end{tabular}

TABLE 10. Base transformation of the character table of $\left\langle s_{i}, s_{j}, s_{k}\right\rangle \cong \Delta(2,2,2)=C_{2} \times C_{2} \times C_{2}, i<j<k$.

A.4.2. $\Delta(2,2, m)$ with $m>2$. This group is isomorphic to $C_{2} \times D_{m}$, and has Coxeter presentation

$$
\Delta(2,2, m)=\left\langle s_{i}, s_{j}, s_{k} \mid s_{i}^{2}, s_{j}^{2}, s_{k}^{2},\left(s_{i} s_{j}\right)^{2},\left(s_{i} s_{k}\right)^{2},\left(s_{j} s_{k}\right)^{m}\right\rangle,
$$

where we have sorted the Coxeter generators $s_{j}$ and $s_{k}$ such that $j<k$ (the generator $s_{i}$ is uniquely determined from the presentation). As a direct product of two groups, the character table of this group can be obtained from those of $C_{2}$ (Table 3) and $D_{m}$ (Table 4). This is shown on Table 11, where $T_{D_{m}}$ is the matrix of entries of the character table of $D_{m}$ (Table 4). As explained before, if $k<j$ one needs to swap the characters $\chi_{3}$ and $\chi_{4}$, that is, swap $\rho_{1} \otimes \chi_{3}$ and $\rho_{1} \otimes \chi_{4}$, and $\rho_{2} \otimes \chi_{3}$ and $\rho_{2} \otimes \chi_{4}$.

The corresponding base transformations for $\Delta(2,2, m) \cong C_{2} \times D_{m}$ are given in Table 12 ( $m$ odd), Table 13 ( $m \geq 6$ even not a power of 2$)$, and Table $14(m \geq 4$ a power of 2). 


\begin{tabular}{|c|c|c|}
\hline$\Delta(2,2, m)$ & $\left(s_{j} s_{k}\right)^{r} \quad s_{k}\left(s_{j} s_{k}\right)^{r}$ & $s_{i}\left(s_{j} s_{k}\right)^{r} \quad s_{i} s_{k}\left(s_{j} s_{k}\right)^{r}$ \\
\hline $\begin{array}{l}\rho_{1} \otimes \chi_{1} \\
\rho_{1} \otimes \chi_{2} \\
\rho_{1} \otimes \widehat{\chi_{3}} \\
\rho_{1} \otimes \widehat{\chi_{4}} \\
\rho_{1} \otimes \phi_{p}\end{array}$ & $T_{D_{m}}$ & $T_{D_{m}}$ \\
\hline $\begin{array}{l}\rho_{2} \otimes \chi_{1} \\
\rho_{2} \otimes \chi_{2} \\
\rho_{2} \otimes \widehat{\chi_{3}} \\
\rho_{2} \otimes \widehat{\chi_{4}} \\
\rho_{2} \otimes \phi_{p}\end{array}$ & $T_{D_{m}}$ & $-T_{D_{m}}$ \\
\hline
\end{tabular}

TABLE 11. Character table of $\Delta(2,2, m), m=m_{j k}>2, j<k$.

\begin{tabular}{c|ccc|ccc}
$D_{m} \times C_{2}$ & $e$ & $s_{i}$ & $\left(s_{i} s_{j}\right)^{r}$ & $\alpha$ & $\alpha s_{i}$ & $\alpha\left(s_{i} s_{j}\right)^{r}$ \\
\hline$\rho_{1} \otimes \chi_{1}$ & 1 & 1 & 1 & 1 & 1 & 1 \\
$\rho_{1} \otimes\left(\chi_{2}-\chi_{1}\right)$ & 0 & -2 & 0 & 0 & -2 & 0 \\
$\rho_{1} \otimes\left(\phi_{1}-\chi_{2}-\chi_{1}\right)$ & 0 & 0 & $b_{r}$ & 0 & 0 & $b_{r}$ \\
$\rho_{1} \otimes\left(\phi_{p}-\phi_{p-1}\right)$, & 0 & 0 & $a_{p, r}$ & 0 & 0 & $a_{p, r}$ \\
\hline$\left(\rho_{2}-\rho_{1}\right) \otimes \chi_{1}$ & 0 & 0 & 1 & -2 & -2 & -2 \\
$\left(\rho_{2}-\rho_{1}\right) \otimes\left(\chi_{2}-\chi_{1}\right)$ & 0 & 0 & 0 & 0 & 4 & 0 \\
$\left(\rho_{2}-\rho_{1}\right) \otimes\left(\phi_{1}-\chi_{2}-\chi_{1}\right)$ & 0 & 0 & 0 & 0 & -4 & $-2 b_{r}$ \\
$\left(\rho_{2}-\rho_{1}\right) \otimes\left(\phi_{p}-\phi_{p-1}\right)$, & 0 & 0 & 0 & 0 & 0 & $-2 a_{p, r}$
\end{tabular}

TABLE 12. Base transformation of the character table of $D_{m} \times C_{2}$ for $m \geq 3$ odd. Here $a_{p, r}:=2 \cos \left(\frac{2 \pi p r}{m}\right)-2 \cos \left(\frac{2 \pi(p-1) r}{m}\right), b_{r}:=$ $2 \cos \left(\frac{2 \pi r}{m}\right)-2,2 \leq p \leq \frac{m-1}{2}$ and $1 \leq r \leq m-1$.

A.4.3. $\Delta(2,3,3)$. This triangle group has Coxeter presentation

$$
\Delta(2,3,3)=\left\langle s_{i}, s_{j}, s_{k} \mid s_{i}^{2}, s_{j}^{2}, s_{k}^{2},\left(s_{i} s_{j}\right)^{3},\left(s_{i} s_{k}\right)^{2},\left(s_{j} s_{k}\right)^{3}\right\rangle .
$$

This group is, in standard notation, $A_{3}$, and it is isomorphic to the symmetric group $S_{4}$, with Coxeter generators $s_{i}=\left(\begin{array}{ll}1 & 2\end{array}\right), s_{j}=\left(\begin{array}{l}2 \\ 2\end{array}\right)$ and $s_{k}=\left(\begin{array}{ll}3 & 4\end{array}\right)$, for instance. Then we have conjugacy classes (cycle types in $S_{4}$ ) represented, in terms of the Coxeter generators, by $s_{i}=\left(\begin{array}{ll}1 & 2\end{array}\right), s_{i} s_{j}=\left(\begin{array}{lll}1 & 3 & 2\end{array}\right), s_{i} s_{j} s_{k}=\left(\begin{array}{llll}1 & 4 & 3 & 2\end{array}\right)$ and $s_{i} s_{k}=\left(\begin{array}{ll}1 & 2\end{array}\right)(34)$. In particular, we have the character table for $\Delta(2,3,3)$ shown in Table 15 below. In this case, the Coxeter generators are unique up to conjugation since Out $\left(S_{4}\right)$ is trivial, hence the choice of $s_{i}$ and $s_{k}$ does not affect the character table.

The required base transformation of the character table Table 15 is given in Table 16.

A.4.4. $\Delta(2,3,4)$. This triangle group has Coxeter presentation

$$
\Delta(2,3,4)=\left\langle s_{i}, s_{j}, s_{k} \mid s_{i}^{2}, s_{j}^{2}, s_{k}^{2},\left(s_{i} s_{j}\right)^{3},\left(s_{i} s_{k}\right)^{2},\left(s_{j} s_{k}\right)^{4}\right\rangle,
$$

and the Coxeter generators are uniquely determined from the presentation. In standard notation, this is the finite Coxeter group $B_{3}$ (or $C_{3}$ ). This group is 


\begin{tabular}{c|ccc|ccc}
$D_{m} \times C_{2}$ & $s_{i}$ & $\left(s_{i} s_{j}\right)^{r}$ & $s_{j} s_{i} s_{j}$ & $\alpha s_{i}$ & $\alpha\left(s_{i} s_{j}\right)^{r}$ & $\alpha s_{j} s_{i} s_{j}$ \\
\hline$\rho_{1} \otimes \chi_{1}$ & 1 & 1 & 1 & 1 & 1 & 1 \\
$\rho_{1} \otimes\left(\chi_{2}-\chi_{1}\right)$ & -2 & 0 & -2 & -2 & 0 & -2 \\
$\rho_{1} \otimes\left(\chi_{3}-\chi_{2}\right)$ & 2 & $c_{r}$ & 0 & 2 & $c_{r}$ & 0 \\
$\rho_{1} \otimes\left(\chi_{4}-\chi_{1}\right)$ & -2 & $c_{r}$ & 0 & -2 & $c_{r}$ & 0 \\
$\rho_{1} \otimes\left(\phi_{1}-\chi_{3}-\chi_{1}\right)$ & -2 & $b_{r}$ & 0 & -2 & $b_{r}$ & 0 \\
$\vdots \rho_{1} \otimes\left(\phi_{p}-\phi_{p-1}\right)$ & & & & & & 0 \\
\hline$\left(\rho_{2}-\rho_{1}\right) \otimes \chi_{1}$ & 0 & $a_{p, r}$ & 0 & 0 & $a_{p, r}$ & -2 \\
$\left(\rho_{2}-\rho_{1}\right) \otimes\left(\chi_{2}-\chi_{1}\right)$ & 0 & 0 & 0 & -2 & -2 & 0 \\
$\left(\rho_{2}-\rho_{1}\right) \otimes\left(\chi_{3}-\chi_{2}\right)$ & 0 & 0 & 0 & -4 & $-2 c_{r}$ & 0 \\
$\left(\rho_{2}-\rho_{1}\right) \otimes\left(\chi_{4}+\chi_{3}-\chi_{2}-\chi_{1}\right)$ & 0 & 0 & 0 & 0 & $-4 c_{r}$ & 0 \\
$\left(\rho_{2}-\rho_{1}\right) \otimes\left(\phi_{1}-\chi_{2}-\chi_{1}\right)$ & 0 & 0 & 0 & 0 & $-2\left(b_{r}+c_{r}\right)$ & 0 \\
$\left(\rho_{2}-\rho_{1}\right) \otimes\left(\phi_{p}-\phi_{p-1}\right)$ & 0 & 0 & 0 & 0 & $-2 a_{p, r}$ & 0
\end{tabular}

TABLE 13. Base transformation of the character table of $D_{m} \times C_{2}$ for $m \geq 6$ even, not a power of 2 . Here $a_{p, r}:=2 \cos \left(\frac{2 \pi p r}{m}\right)-$ $2 \cos \left(\frac{2 \pi(p-1) r}{m}\right), b_{r}:=2 \cos \left(\frac{2 \pi r}{m}\right)-(-1)^{r}-1, c_{r}=(-1)^{r}-1$ and $1<p, r<\frac{m}{2}$.

\begin{tabular}{|c|c|c|c|c|c|c|}
\hline$D_{m} \times C_{2}$ & $s_{i}$ & $\left(s_{i} s_{j}\right)^{r}$ & $s_{j} s_{i} s_{j}$ & $\alpha s_{i}$ & $\alpha\left(s_{i} s_{j}\right)^{r}$ & $\alpha s_{j} s_{i} s_{j}$ \\
\hline$\rho_{1} \otimes \chi_{1}$ & 1 & 1 & 1 & 1 & 1 & 1 \\
\hline$\rho_{1} \otimes\left(\chi_{2}-\chi_{1}\right)$ & -2 & 0 & -2 & -2 & 0 & -2 \\
\hline$\rho_{1} \otimes\left(\chi_{3}-\chi_{2}\right)$ & 0 & $c_{r}$ & -2 & 0 & $c_{r}$ & -2 \\
\hline$\rho_{1} \otimes\left(\chi_{4}-\chi_{1}\right)$ & 0 & $c_{r}$ & 2 & 0 & $c_{r}$ & 2 \\
\hline$\rho_{1} \otimes\left(\phi_{1}-\chi_{3}-\chi_{1}\right)$ & 0 & $b_{r}$ & 0 & 0 & $b_{r}$ & 0 \\
\hline$\vdots \quad \rho_{1} \otimes\left(\phi_{p}-\phi_{p-1}\right) \quad \vdots$ & 0 & $a_{p, r}$ & 0 & 0 & $a_{p, r}$ & 0 \\
\hline$\left(\rho_{2}-\rho_{1}\right) \otimes \chi_{1}$ & 0 & 0 & 0 & -2 & -2 & -2 \\
\hline$\left(\rho_{2}-\rho_{1}\right) \otimes\left(\chi_{2}-\chi_{1}\right)$ & 0 & 0 & 0 & 4 & 0 & 4 \\
\hline$\left(\rho_{2}-\rho_{1}\right) \otimes\left(\chi_{3}-\chi_{2}\right)$ & 0 & 0 & 0 & 0 & $-2 c_{r}$ & 4 \\
\hline$\left(\rho_{2}-\rho_{1}\right) \otimes\left(\chi_{4}+\chi_{3}-\chi_{2}-\chi_{1}\right)$ & 0 & 0 & 0 & 0 & $-4 c_{r}$ & 0 \\
\hline$\left(\rho_{2}-\rho_{1}\right) \otimes\left(\phi_{1}-\chi_{2}-\chi_{1}\right)$ & 0 & 0 & 0 & 0 & $-2 b_{r}$ & 0 \\
\hline$\left(\rho_{2}-\rho_{1}\right) \otimes\left(\phi_{p}-\phi_{p-1}\right)$ & 0 & 0 & 0 & 0 & $-2 a_{p, r}$ & 0 \\
\hline
\end{tabular}

TABLE 14. Base transformation of the character table of $D_{m} \times$ $C_{2}$ for $m \geq 4$ even a power of 2 . Here $a_{p, r}:=2 \cos \left(\frac{2 \pi p r}{m}\right)-$ $2 \cos \left(\frac{2 \pi(p-1) r}{m}\right), b_{r}:=2 \cos \left(\frac{2 \pi r}{m}\right)-2, c_{r}=(-1)^{r}-1$ and $1<p, r<$ $\frac{m}{2}$.

isomorphic to $S_{4} \times C_{2}$ with Coxeter generators, for instance, $s_{i}=\left(\begin{array}{ll}12 \\ 2\end{array}\right) \alpha, s_{j}=\left(\begin{array}{ll}13 \\ \text { ) } \alpha\end{array}\right.$ and $s_{k}=\left(\begin{array}{lll}1 & 2\end{array}\right)\left(\begin{array}{ll}3 \\ 4\end{array}\right) \alpha$, where $\alpha$ is the generator of the $C_{2}$ factor. We can choose representatives of the conjugacy classes in terms of the Coxeter generators, for 


\begin{tabular}{c|ccccc}
$\Delta(2,3,3)$ & $e$ & $s_{i}$ & $s_{i} s_{j}$ & $s_{i} s_{j} s_{k}$ & $s_{i} s_{k}$ \\
\hline$\xi_{1}$ & 1 & 1 & 1 & 1 & 1 \\
$\xi_{2}$ & 1 & -1 & 1 & -1 & 1 \\
$\xi_{3}$ & 2 & 0 & -1 & 0 & 2 \\
$\xi_{4}$ & 3 & 1 & 0 & -1 & -1 \\
$\xi_{5}$ & 3 & -1 & 0 & 1 & -1
\end{tabular}

TABLE 15. Character table of $\left\langle s_{i}, s_{j}, s_{k}\right\rangle \cong \Delta(2,3,3) \cong S_{4}$.

\begin{tabular}{c|ccccc}
$S_{4}$ & $e$ & $s_{i}$ & $s_{i} s_{j}$ & $s_{i} s_{j} s_{k}$ & $s_{i} s_{k}$ \\
\hline$\xi_{1}$ & 1 & 1 & 1 & 1 & 1 \\
$\widetilde{\xi_{2}}:=\xi_{2}-\xi_{1}$ & 0 & -2 & 0 & -2 & 0 \\
$\widetilde{\xi}_{3}:=\xi_{3}-\xi_{2}-\xi_{1}$ & 0 & 0 & -3 & 0 & 0 \\
$\widetilde{\xi_{4}}:=\xi_{4}-\xi_{3}-\xi_{1}$ & 0 & 0 & 0 & -2 & -4 \\
$\widetilde{\xi_{5}}:=\xi_{5}-\xi_{4}-\xi_{2}+\xi_{1}$ & 0 & 0 & 0 & 4 & 0
\end{tabular}

TABLE 16. Base transformation of the character table of $\Delta(2,3,3) \cong S_{4}$.

example,

$$
\begin{aligned}
& e \sim e \\
& \alpha \sim\left(s_{i} s_{j} s_{k}\right)^{3} \\
& (12) \sim s_{i} s_{k} \\
& (12) \alpha \sim s_{i} \\
& \left(\begin{array}{lll}
1 & 2 & 3
\end{array}\right) \sim s_{i} s_{j} \\
& \text { (1 } 23) \alpha \sim s_{i} s_{j} s_{k} \\
& \left(\begin{array}{lll}
1 & 2 & 3
\end{array}\right) \sim s_{j} s_{k} \\
& \text { (1 } 2334) \alpha \sim s_{i}\left(s_{j} s_{k}\right)^{2} \\
& (12)(34) \sim\left(s_{j} s_{k}\right)^{2} \\
& \text { (1 2)(34) } \alpha \sim s_{k}
\end{aligned}
$$

where ' $\sim$ ' means 'conjugate'. Using this representatives, and the fact that $\Delta(2,3,4)$ is a direct product, the character table of this group can be written as in Table 17 below, where $T_{S_{4}}$ is the matrix of coefficients of the character table of $S_{4}$ (Table 15).

The base transformation needed is given in Table 18 , where $\left\{\widetilde{\xi}_{i}\right\}$ is the transformed basis of the character table of $S_{4}$, Table 16 .

A.4.5. $\Delta(2,3,5)$. This group has Coxeter presentation

$$
\Delta(2,3,5)=\left\langle s_{i}, s_{j}, s_{k} \mid s_{i}^{2}, s_{j}^{2}, s_{k}^{2},\left(s_{i} s_{j}\right)^{3},\left(s_{i} s_{k}\right)^{2},\left(s_{j} s_{k}\right)^{5}\right\rangle
$$

and it is isomorphic to $A_{5} \times C_{2}$ with Coxeter generators $s_{i}=(12)(35) \alpha, s_{j}=$ (1 2) $\left(\begin{array}{ll}3 & 4\end{array}\right) \alpha, s_{k}=\left(\begin{array}{ll}1 & 5\end{array}\right)\left(\begin{array}{ll}2 & 3\end{array}\right) \alpha$, for example. In standard notation, this is the exceptional finite Coxeter group $\mathrm{H}_{3}$.

Remark 3. These Coxeter generators are not unique, since $\operatorname{Out}\left(A_{5} \times C_{2}\right) \cong C_{2}$. There are then two sets of Coxeter generators up to conjugation, the other one given by conjugation by a suitable $g \in S_{5} \backslash A_{5}$, for instance, conjugating by $g=(24)$ : $s_{i}^{\prime}=\left(\begin{array}{ll}1 & 4\end{array}\right)\left(\begin{array}{ll}3 & 5\end{array}\right) \alpha, s_{j}^{\prime}=\left(\begin{array}{ll}1 & 4\end{array}\right)\left(\begin{array}{ll}2 & 3\end{array}\right) \alpha, s_{k}^{\prime}=\left(\begin{array}{ll}1 & 5\end{array}\right)\left(\begin{array}{ll}3 & 4\end{array}\right) \alpha$. In the first case $s_{j} s_{k}=$ 


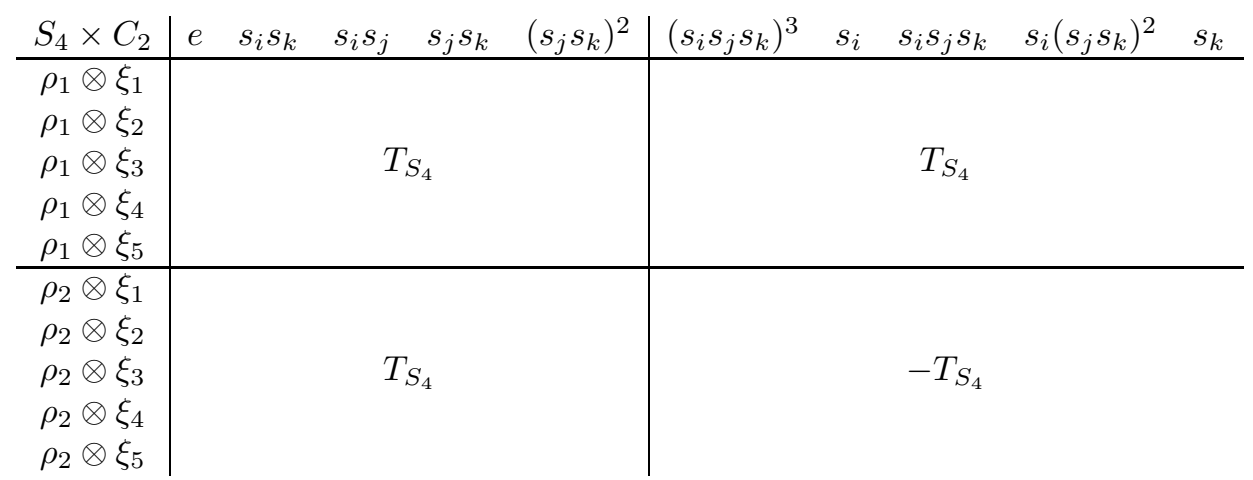

TABLE 17. Character table of $\left\langle s_{i}, s_{j}, s_{k}\right\rangle \cong \Delta(2,3,4)=S_{4} \times C_{2}$.

\begin{tabular}{c|ccccc|ccccc}
$S_{4} \times C_{2}$ & $(1)$ & $(12)$ & $(123)$ & $(1234)$ & $(12)(34)$ & $\alpha$ & $\alpha(12)$ & $\alpha(123)$ & $\alpha(1234)$ & $\alpha(12)(34)$ \\
\hline$\alpha_{1}$ & 1 & 1 & 1 & 1 & 1 & 1 & 1 & 1 & 1 & 1 \\
$\alpha_{2}$ & 0 & -2 & 0 & -2 & 0 & 0 & -2 & 0 & -2 & 0 \\
$\alpha_{3}$ & 0 & 0 & -3 & 0 & 0 & 0 & 0 & -3 & 0 & 0 \\
$\alpha_{4}$ & 0 & -2 & 0 & 4 & -4 & 2 & 0 & 2 & 6 & -2 \\
$\alpha_{5}$ & 0 & 0 & 0 & 4 & 0 & 0 & 0 & 0 & 4 & 0 \\
\hline$\alpha_{6}$ & 0 & 2 & 0 & 2 & 0 & -2 & 0 & -2 & 0 & -2 \\
$\alpha_{7}$ & 0 & -4 & 0 & 0 & 0 & 0 & 0 & 0 & 4 & 0 \\
$\alpha_{8}$ & 0 & 0 & 0 & 0 & 0 & 0 & 0 & 6 & 0 & 0 \\
$\alpha_{9}$ & 0 & 0 & 0 & 6 & -4 & 4 & 0 & 4 & -6 & 8 \\
$\alpha_{10}$ & 0 & 0 & 0 & 0 & 0 & 0 & 0 & 0 & -8 & 0
\end{tabular}

where $\alpha_{1}:=\rho_{1} \otimes \xi_{1}, \alpha_{2}:=\rho_{1} \otimes \widetilde{\xi_{2}}, \alpha_{3}:=\rho_{1} \otimes \widetilde{\xi_{3}}, \alpha_{4}:=\rho_{1} \otimes\left(\widetilde{\xi_{4}}+2 \widetilde{\xi_{5}}\right)-\left(\rho_{2} \otimes \xi_{1}-\rho_{1} \otimes \xi_{2}\right)$, $\alpha_{5}:=\rho_{1} \otimes \widetilde{\xi}_{5}, \alpha_{6}:=\rho_{2} \otimes \xi_{1}-\rho_{1} \otimes \xi_{2}, \alpha_{7}:=\rho_{2} \otimes \xi_{2}-\rho_{2} \otimes \xi_{1}+\rho_{1} \otimes\left(\xi_{5}-\xi_{4}\right)$, $\alpha_{8}:=\left(\rho_{2}-\rho_{1}\right) \otimes \widetilde{\xi_{3}}, \alpha_{9}:=\rho_{2} \otimes\left(\widetilde{\xi_{4}}+2 \widetilde{\xi_{5}}\right)+\left(\rho_{1}-\rho_{2}\right) \otimes\left(\xi_{1}+\xi_{2}\right), \alpha_{10}:=\left(\rho_{2}-\rho_{1}\right) \otimes \widetilde{\xi_{5}}$.

TABLE 18. Base transformation of the character table of $\left\langle s_{i}, s_{j}, s_{k}\right\rangle \cong \Delta(2,3,4)=S_{4} \times C_{2}$.

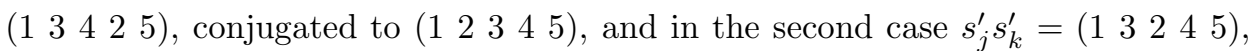
conjugated to (1 32245 ), which represent different conjugacy classes in $A_{5}$.

A character table for the alternating group $A_{5}$ reads as follows.

\begin{tabular}{|c|c|c|c|c|c|}
\hline$A_{5}$ & $e$ & $\left(\begin{array}{lll}1 & 2 & 3\end{array}\right)$ & $\left(\begin{array}{lll}1 & 2\end{array}\right)(34)$ & 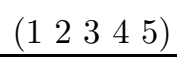 & $\left(\begin{array}{llll}1 & 3 & 4 & 5\end{array}\right)$ \\
\hline$\xi_{1}$ & 1 & 1 & 1 & 1 & 1 \\
\hline$\xi_{2}$ & 4 & 1 & 0 & -1 & -1 \\
\hline$\xi_{3}$ & 5 & -1 & 1 & 0 & 0 \\
\hline$\xi_{4}$ & 3 & 0 & -1 & $\frac{1+\sqrt{5}}{2}$ & $\frac{1-\sqrt{5}}{2}$ \\
\hline$\varepsilon_{-}$ & 3 & 0 & -1 & $\frac{1-\sqrt{5}}{2}$ & $\frac{1+\sqrt{5}}{2}$ \\
\hline
\end{tabular}

If we call $T_{A_{5}}$ the matrix of coefficients of this table then the character table of $\Delta(2,3,5)$ is given by Table 19 , where we have used the following representatives of 
the conjugacy classes in terms of the Coxeter generators (' $\sim$ ' means 'conjugate')

$$
e \sim e \quad \alpha \sim\left(s_{i} s_{j} s_{k}\right)^{5}
$$

$$
\left(\begin{array}{lll}
1 & 2 & 3
\end{array}\right) \sim s_{i} s_{j} \quad(123) \alpha \sim s_{i}\left(s_{j} s_{k}\right)^{2}
$$

$(12)(34) \sim s_{i} s_{k}$

(1 2) (34) $\alpha \sim s_{i}$

$(12345) \sim s_{j} s_{k}$

$\left(\begin{array}{llll}1 & 2 & 3 & 4\end{array}\right) \alpha \sim s_{i} s_{j} s_{k}$

\begin{tabular}{|c|c|c|c|c|c|c|c|}
\hline$A_{5} \times C_{2}$ & $e \quad s_{i} s_{j}$ & $s_{i} s_{k} \quad s_{j} s_{k}$ & $\left(s_{i} s_{j} s_{k}\right)^{4}$ & $\left(s_{i} s_{j} s_{k}\right)^{5}$ & $s_{i}\left(s_{j} s_{k}\right)^{2} \quad s_{i}$ & $s_{i} s_{j} s_{k}$ & $s_{j} s_{i} s_{k}$ \\
\hline $\begin{array}{l}\rho_{1} \otimes \xi_{1} \\
\rho_{1} \otimes \xi_{2} \\
\rho_{1} \otimes \xi_{3} \\
\rho_{1} \otimes \xi_{4} \\
\rho_{1} \otimes \xi_{5}\end{array}$ & & $T_{A_{5}}$ & & & $T_{A_{5}}$ & & \\
\hline $\begin{array}{l}\rho_{2} \otimes \xi_{1} \\
\rho_{2} \otimes \xi_{2} \\
\rho_{2} \otimes \xi_{3} \\
\rho_{2} \otimes \xi_{4} \\
\rho_{2} \otimes \xi_{5}\end{array}$ & & $T_{A_{5}}$ & & & $-T_{A_{5}}$ & & \\
\hline
\end{tabular}

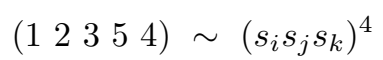

$(12354) \alpha \sim s_{j} s_{i} s_{k}$

TABLE 19. Character table of $\left\langle s_{i}, s_{j}, s_{k}\right\rangle \cong \Delta(2,3,5)=A_{5} \times C_{2}$.

Remark 4. With the non-conjugate set of Coxeter generators $s_{i}^{\prime}, s_{j}^{\prime}, s_{k}^{\prime}$ (see Remark 3 ), we get the analogous set of representatives except swapping the obvious ones, that is, $s_{j} s_{k} \sim\left(s_{i}^{\prime} s_{j}^{\prime} s_{k}^{\prime}\right)^{4},\left(s_{i} s_{j} s_{k}\right)^{4} \sim s_{j}^{\prime} s_{k}^{\prime},\left(s_{i} s_{j} s_{k}\right)^{5} s_{j} s_{k} \sim s_{i}^{\prime} s_{j}^{\prime} s_{k}^{\prime}$ and $s_{i} s_{j} s_{k} \sim$ $\left(s_{i}^{\prime} s_{j}^{\prime} s_{k}^{\prime}\right)^{5} s_{j}^{\prime} s_{k}^{\prime}$. On the character table this amounts to interchanging $\rho_{i} \otimes \xi_{4}$ with $\rho_{i} \otimes \xi_{5}$ for $i=1$ and 2 .

\begin{tabular}{|c|c|c|c|c|c|}
\hline$A_{5}$ & $e$ & $\left(\begin{array}{lll}1 & 2 & 3\end{array}\right)$ & $\left(\begin{array}{lll}1 & 2\end{array}\right)\left(\begin{array}{ll}3 & 4\end{array}\right)$ & $\left(\begin{array}{lllll}1 & 2 & 3 & 4 & 5\end{array}\right)$ & $\left(\begin{array}{lllll}1 & 3 & 4 & 5 & 2\end{array}\right)$ \\
\hline$\xi_{1}$ & 1 & 1 & 1 & 1 & 1 \\
\hline$\widetilde{\xi_{2}}:=\xi_{2}-4 \xi_{1}$ & 0 & -3 & -4 & -5 & -5 \\
\hline$\widetilde{\xi_{3}}:=\xi_{3}-\xi_{2}-\xi_{1}$ & 0 & -3 & 0 & 0 & 0 \\
\hline$\widetilde{\xi_{4}}:=\xi_{4}-\xi_{2}+\xi_{1}$ & 0 & 0 & 0 & $\frac{5+\sqrt{5}}{2}$ & $\frac{5-\sqrt{5}}{2}$ \\
\hline$\widetilde{\xi}_{5}:=\xi_{5}+\xi_{4}-\xi_{1}-\xi_{3}$ & 0 & 0 & -4 & 0 & 0 \\
\hline
\end{tabular}

For the base transformation required, first note that the character table for the alternating group $A_{5}$ above can be transformed as follows,

\begin{tabular}{|c|c|c|c|c|c|}
\hline$A_{5}$ & $e$ & $\left(\begin{array}{lll}1 & 2 & 3\end{array}\right)$ & $\left(\begin{array}{ll}1 & 2\end{array}\right)\left(\begin{array}{ll}3 & 4\end{array}\right)$ & $\left(\begin{array}{lllll}1 & 2 & 3 & 4 & 5\end{array}\right)$ & $\left(\begin{array}{lllll}1 & 3 & 4 & 5 & 2\end{array}\right)$ \\
\hline$\xi_{1}$ & 1 & 1 & 1 & 1 & 1 \\
\hline$\widetilde{\xi_{2}}:=\widetilde{\xi_{2}}-\widetilde{\xi_{3}}-\widetilde{\xi_{5}}$ & 0 & 0 & 0 & -5 & -5 \\
\hline$\widetilde{\xi}_{3}$ & 0 & -3 & 0 & 0 & 0 \\
\hline$\widetilde{\xi_{4}}$ & 0 & 0 & 0 & $\frac{5+\sqrt{5}}{2}$ & $\frac{5-\sqrt{5}}{2}$ \\
\hline$\widetilde{\xi}_{5}$ & 0 & 0 & -4 & 0 & 0 \\
\hline
\end{tabular}

and then

With this notation, the required base transformation is given in Table 20 . 


\begin{tabular}{c|ccccc|ccccc}
$A_{5} \times C_{2}$ & $e$ & $s_{i} s_{j}$ & $s_{i} s_{k}$ & $s_{j} s_{k}$ & $\left(s_{i} s_{j} s_{k}\right)^{4}$ & $\left(s_{i} s_{j} s_{k}\right)^{5}$ & $s_{i}\left(s_{j} s_{k}\right)^{2}$ & $s_{i}$ & $s_{i} s_{j} s_{k}$ & $s_{j} s_{i} s_{k}$ \\
\hline$\beta_{1}$ & 1 & 1 & 1 & 1 & 1 & 1 & 1 & 1 & 1 & 1 \\
$\beta_{2}$ & 0 & 0 & 0 & $\sqrt{5}$ & $-\sqrt{5}$ & 0 & 0 & 0 & $\sqrt{5}$ & $-\sqrt{5}$ \\
$\beta_{3}$ & 0 & -3 & 0 & 0 & 0 & 0 & -3 & 0 & 0 & 0 \\
$\beta_{4}$ & 0 & 0 & 0 & $\frac{5+\sqrt{5}}{2}$ & $\frac{5-\sqrt{5}}{2}$ & 0 & 0 & 0 & $\frac{5+\sqrt{5}}{2}$ & $\frac{5-\sqrt{5}}{2}$ \\
$\beta_{5}$ & 0 & 0 & -4 & 0 & 0 & 4 & 4 & 0 & 4 & 4 \\
\hline$\beta_{6}$ & 0 & 0 & 0 & 0 & 0 & -2 & -2 & -2 & -2 & -2 \\
$\beta_{7}$ & 0 & 0 & 0 & 0 & 0 & 0 & 0 & 0 & 10 & 10 \\
$\beta_{8}$ & 0 & 0 & 0 & 0 & 0 & 0 & 6 & 0 & 0 & 0 \\
$\beta_{9}$ & 0 & 0 & 0 & 0 & 0 & 0 & 0 & 0 & $-5-\sqrt{5}$ & $-5+\sqrt{5}$ \\
$\beta_{10}$ & 0 & 0 & 0 & 0 & 0 & -8 & -8 & 0 & -8 & -8
\end{tabular}

where $\beta_{1}:=\rho_{1} \otimes \xi_{1}, \beta_{2}:=\rho_{1} \otimes \widetilde{\xi_{2}}+2 \rho_{1} \otimes \widetilde{\xi_{4}}, \beta_{3}:=\rho_{1} \otimes \widetilde{\xi_{3}}, \beta_{4}:=\rho_{1} \otimes \widetilde{\xi_{4}}$, $\beta_{5}:=\rho_{1} \otimes \widetilde{\xi_{5}}-2\left(\rho_{2}-\rho_{1}\right) \otimes \xi_{1}, \beta_{6}:=\left(\rho_{2}-\rho_{1}\right) \otimes \xi_{1}, \beta_{7}:=\left(\rho_{2}-\rho_{1}\right) \otimes \widetilde{\xi_{2}}, \beta_{8}:=\left(\rho_{2}-\rho_{1}\right) \otimes \widetilde{\xi_{3}}$, $\beta_{9}:=\left(\rho_{2}-\rho_{1}\right) \otimes \widetilde{\xi_{4}}, \beta_{10}:=\left(\rho_{2}-\rho_{1}\right) \otimes\left(\widetilde{\xi_{5}}+4 \xi_{1}\right)$.

TABLE 20. Base transformation of the character table of $\left\langle s_{i}, s_{j}, s_{k}\right\rangle \cong \Delta(2,3,5)=A_{5} \times C_{2}$.

\section{ApPENDix B. INDUCTION HOMOMORPHISMS}

In this Appendix, we compute all possible induction homomorphisms $R_{\mathbb{C}}(H) \rightarrow$ $R_{\mathbb{C}}(G)$ appearing in the Bredon chain complex (6). That is, $G$ is a finite Coxeter subgroup of $\Gamma$ of rank $n$ generated by $n \leq 3$ of the Coxeter generators (1), and $H$ is a subgroup of $G$ generated by a subset of exactly $n-1$ of those Coxeter generators.

We give explicit induction homomorphisms with respect to the standard character tables, and also with respect to the transformed bases in Appendix A for rank 3 subgroups, as this is needed for the simultaneous base transformation argument in the proof that $\mathrm{H}_{0}^{\mathfrak{F} \mathfrak{i n}}\left(\Gamma ; R_{\mathbb{C}}\right)$ is torsion-free (Section 4).

We implicitly use the character tables and notation in Appendix A, and Frobenius reciprocity [23], throughout this Appendix. We also note that Frobenius reciprocity extends linearly:

Lemma 3. If $H$ is a subgroup of a finite group $G$ and $\phi$ and $\eta$, respectively $\tau$ and $\pi$, are representations of $G$, respectively $H$, then

$$
(\phi \downarrow+\xi \downarrow \mid \tau+\pi)=(\phi+\xi \mid \tau \uparrow+\pi \uparrow) .
$$

Proof.

$$
\begin{aligned}
(\phi \downarrow+\xi \downarrow \mid \tau+\pi) & =\frac{1}{|H|} \sum_{h \in H}(\phi \downarrow+\xi \downarrow)(h) \cdot \overline{(\tau+\pi)(h)} \\
& =\frac{1}{|H|} \sum_{h \in H}(\phi \downarrow \cdot \bar{\tau}+\xi \downarrow \cdot \bar{\tau}+\phi \downarrow \cdot \bar{\pi}+\xi \downarrow \cdot \bar{\pi})(h)
\end{aligned}
$$

which by Frobenius reciprocity on irreducible characters equals

$$
\frac{1}{|G|} \sum_{g \in G}(\phi \cdot \bar{\tau} \uparrow+\xi \cdot \bar{\tau} \uparrow+\phi \cdot \bar{\pi} \uparrow+\xi \cdot \bar{\pi} \uparrow)(h)=(\phi+\xi \mid \tau \uparrow+\pi \uparrow) .
$$

B.1. Rank 1. The only induction homomorphism in this case is

$$
R_{\mathbb{C}}(\{e\}) \rightarrow R_{\mathbb{C}}\left(\left\langle s_{i}\right\rangle\right)
$$


which must the regular representation $\tau \mapsto \rho_{1}+\rho_{2}$ shown, in terms of free abelian groups, in Figure 2.

$$
\begin{aligned}
R_{\mathbb{C}}(\{e\}) & \rightarrow R_{\mathbb{C}}\left(C_{2}\right) \\
\mathbb{Z} & \rightarrow \mathbb{Z}^{2} \\
a & \mapsto(a, a)
\end{aligned}
$$

FiguRE 2. Induction homomorphism from $H=\{e\}$ to $G=\left\langle s_{i}\right\rangle \cong C_{2}$.

B.2. Rank 2. In this case $G$ is a dihedral group with the presentation

$$
G=\left\langle s_{i}, s_{j} \mid s_{i}^{2}=s_{j}^{2}=\left(s_{i} s_{j}\right)^{m}\right\rangle,
$$

where $m=m_{i j}$ and we assume $i<j$. Consider first the case $H=\left\langle s_{i}\right\rangle$. The characters of $D_{m}$ (Table 5) restricted to $H$ are (note that $s_{i}=s_{j}\left(s_{i} s_{j}\right)^{m-1}$ )

\begin{tabular}{c|rr}
$D_{m} \downarrow$ & $e$ & $s_{i}$ \\
\hline$\chi_{1}$ & 1 & 1 \\
$\chi_{2}$ & 1 & -1 \\
$\widetilde{\chi_{3}}$ & 1 & -1 \\
$\widehat{\chi_{4}}$ & 1 & 1 \\
$\phi_{p}$ & 2 & 0
\end{tabular}

Multiplying with the rows of the character table of $H \cong C_{2}$ (Table 3) we obtain the induced representations

$$
\begin{aligned}
& \rho_{1} \uparrow=\chi_{1}+\widehat{\chi_{4}}+\sum \phi_{p}, \\
& \rho_{2} \uparrow=\chi_{2}+\widehat{\chi_{3}}+\sum \phi_{p} .
\end{aligned}
$$

The other case is when $H=\left\langle s_{j}\right\rangle$. This is analogous, but note that, in order to keep the notation consistent with Table 4 , the characters $\chi_{3}$ and $\chi_{4}$ must be interchanged in the even case. Specifically, we have now $s_{j}=s_{j}\left(s_{i} s_{j}\right)^{0}$ and hence

\begin{tabular}{c|rr}
$D_{m} \downarrow$ & $e$ & $s_{j}$ \\
\hline$\chi_{1}$ & 1 & 1 \\
$\chi_{2}$ & 1 & -1 \\
$\widetilde{\chi_{3}}$ & 1 & 1 \\
$\widetilde{\chi_{4}}$ & 1 & -1 \\
$\phi_{p}$ & 2 & 0
\end{tabular}

and therefore

$$
\begin{aligned}
& \rho_{1} \uparrow=\chi_{1}+\widehat{\chi_{3}}+\sum \phi_{p}, \\
& \rho_{2} \uparrow=\chi_{2}+\widehat{\chi_{4}}+\sum \phi_{p} .
\end{aligned}
$$

All in all, as maps of free abelian groups, we have that the induction homomorphisms $R_{\mathbb{C}}(H) \rightarrow R_{\mathbb{C}}(G)$ shown in Figure 3 .

B.3. Rank 3. We compute each case individually. 


$$
\begin{aligned}
& R_{\mathbb{C}}(H) \rightarrow R_{\mathbb{C}}(G) \\
& \mathbb{Z}^{2} \rightarrow \mathbb{Z}^{c\left(D_{m}\right)} \\
& (a, b) \mapsto(a, b, \widehat{b}, \widehat{a}, a+b, \ldots, a+b) \text { for } H=\left\langle s_{i}\right\rangle, \\
& (a, b) \mapsto \quad(a, b, \widehat{a}, \widehat{b}, a+b, \ldots, a+b) \quad \text { for } H=\left\langle s_{j}\right\rangle,
\end{aligned}
$$

FiguRE 3. Induction homomorphisms from $H$ to $G=\left\langle s_{i}, s_{j}\right\rangle \cong$ $D_{m}, m=m_{i j}$ and $i<j$.

B.3.1. $G=\Delta(2,2,2)$. This group is isomorphic to $C_{2} \times C_{2} \times C_{2}$, and has Coxeter generators $s_{i}, s_{j}, s_{k}$ where $i<j<k$. We compute the induction homomorphisms for the Coxeter subgroups $\left\langle s_{i}, s_{j}\right\rangle,\left\langle s_{i}, s_{k}\right\rangle$ and $\left\langle s_{j}, s_{k}\right\rangle$, all three direct factors of $G$ and isomorphic to $C_{2} \times C_{2}$. Using the bases of $R_{\mathbb{C}}\left(C_{2} \times C_{2}\right)$ and $R_{\mathbb{C}}(\Delta(2,2,2))$ induced from $C_{2}$ (Tables 5 and 9), we immediately obtain the induction homomorphisms shown, as maps of free abelian groups, shown in Figure 4.

Remark 5. Recall how to the induction homomorphism works from a group $A$ to direct product $A \times B$ : if $\rho$ is a representation of $A$ then $\operatorname{Ind}_{A}^{A \times B}(\rho)=\rho \otimes r_{B}$, where $r_{B}$ is the regular representation of $B$.

$$
\begin{array}{rll}
R_{\mathbb{C}}(H) & \rightarrow R_{\mathbb{C}}(G) & \\
\mathbb{Z}^{4} & \rightarrow \mathbb{Z}^{8} & \\
(a, b, c, d) & \mapsto(a, a, b, b, c, c, d, d) & \text { for } H=\left\langle s_{i}, s_{j}\right\rangle, \\
(a, b, c, d) & \mapsto(a, b, a, b, c, d, c, d) & \text { for } H=\left\langle s_{i}, s_{k}\right\rangle, \\
(a, b, c, d) & \mapsto(a, b, c, d, a, b, c, d) & \text { for } H=\left\langle s_{j}, s_{k}\right\rangle .
\end{array}
$$

FIgURE 4. Induction homomorphisms from $H$ to $G=$ $\left\langle s_{i}, s_{j}, s_{k}\right\rangle \cong \Delta(2,2,2)=C_{2} \times C_{2} \times C_{2}$, and $i<j<k$.

On the other hand, with respect to the transformed bases (Tables 6 and 10 in Appendix A), the induction homomorphisms take the form shown in Tables 21, 22, and 23. Note that, for the transformed bases, we show the restricted characters, and the induced map, in two adjacent tables separated by double vertical bars.

B.3.2. $G=\Delta(2,2, m), m>2$. This group is isomorphic to $C_{2} \times D_{m}$ with Coxeter presentation

$$
\Delta(2,2, m)=\left\langle s_{i}, s_{j}, s_{k} \mid s_{i}^{2}, s_{j}^{2}, s_{k}^{2},\left(s_{i} s_{j}\right)^{2},\left(s_{i} s_{k}\right)^{2},\left(s_{j} s_{k}\right)^{m}\right\rangle,
$$

We have three relevant Coxeter subgroups $H$, which we treat separately. In each case, we restrict the characters of $G$ (Table 11) to the subgroup $H$ and then use Frobenius reciprocity to write the induced characters of $H$ into $G$ in terms of the characters of $G$.

Case 1: $H=\left\langle s_{i}, s_{j}\right\rangle \cong D_{2}=C_{2} \times C_{2}$.

The elements $e, s_{i}, s_{j}$ and $s_{i} s_{j}$ of $H$ are obtained from the 1st, 3rd, 2nd and 4th 


\begin{tabular}{c|rrrr||rrrr}
$\left\langle s_{j}, s_{k}\right\rangle \hookrightarrow C_{2} \times C_{2} \times C_{2}$ & $e$ & $s_{j}$ & $s_{k}$ & $s_{j} s_{k}$ & $\left(\cdot \mid \sum \chi_{i}\right)$ & $\left(\cdot \mid \chi_{2}+\chi_{3}\right)$ & $\left(\cdot \mid \chi_{3}+\chi_{4}\right)$ & $\left(\cdot \mid \chi_{4}\right)$ \\
\hline$\rho_{111} \downarrow$ & 1 & 1 & 1 & 1 & 1 & 0 & 0 & 0 \\
$\rho_{112}-\rho_{111} \downarrow$ & 0 & 0 & -2 & -2 & 0 & 1 & 0 & 0 \\
$\rho_{121}-\rho_{111} \downarrow$ & 0 & -2 & 0 & -2 & 0 & 0 & 1 & 1 \\
$\rho_{122}-\rho_{121} \downarrow$ & 0 & 0 & -2 & 2 & 0 & 1 & 0 & -1 \\
$\rho_{211}-\rho_{111} \downarrow$ & 0 & 0 & 0 & 0 & 0 & 0 & 0 & 0 \\
$\rho_{212}-\rho_{211} \downarrow$ & 0 & 0 & -2 & -2 & 0 & 1 & 0 & 0 \\
$\rho_{221}-\rho_{121} \downarrow$ & 0 & 0 & 0 & 0 & 0 & 0 & 0 & 0 \\
$\rho_{222}-\rho_{221} \downarrow$ & 0 & 0 & -2 & 2 & 0 & 1 & 0 & -1
\end{tabular}

TABLE 21. Restricted characters and induced map $\left\langle s_{j}, s_{k}\right\rangle \hookrightarrow$ $\Delta(2,2,2)=C_{2} \times C_{2} \times C_{2}$.

\begin{tabular}{c|rrrr||rrrr}
$\left\langle s_{i}, s_{k}\right\rangle \hookrightarrow C_{2} \times C_{2} \times C_{2}$ & $e$ & $s_{i}$ & $s_{k}$ & $s_{i} s_{k}$ & $\left(\cdot \mid \sum \chi_{i}\right)$ & $\left(\cdot \mid \chi_{2}+\chi_{3}\right)$ & $\left(\cdot \mid \chi_{3}+\chi_{4}\right)$ & $\left(\cdot \mid \chi_{4}\right)$ \\
\hline$\rho_{111} \downarrow$ & 1 & 1 & 1 & 1 & 1 & 0 & 0 & 0 \\
$\rho_{112}-\rho_{111} \downarrow$ & 0 & 0 & -2 & -2 & 0 & 1 & 0 & 0 \\
$\rho_{121}-\rho_{111} \downarrow$ & 0 & 0 & 0 & 0 & 0 & 0 & 0 & 0 \\
$\rho_{122}-\rho_{121} \downarrow$ & 0 & 0 & -2 & -2 & 0 & 1 & 0 & 0 \\
$\rho_{211}-\rho_{111} \downarrow$ & 0 & -2 & 0 & -2 & 0 & 0 & 1 & 1 \\
$\rho_{212}-\rho_{211} \downarrow$ & 0 & 0 & -2 & 2 & 0 & 1 & 0 & -1 \\
$\rho_{221}-\rho_{121} \downarrow$ & 0 & -2 & 0 & -2 & 0 & 0 & 1 & 1 \\
$\rho_{222}-\rho_{221} \downarrow$ & 0 & 0 & -2 & 2 & 0 & 1 & 0 & -1
\end{tabular}

TABLE 22. Restricted characters and induced map $\left\langle s_{i}, s_{k}\right\rangle \hookrightarrow$ $\Delta(2,2,2)=C_{2} \times C_{2} \times C_{2}$.

\begin{tabular}{c|rrrr||rrrr}
$\left\langle s_{i}, s_{j}\right\rangle \hookrightarrow C_{2} \times C_{2} \times C_{2}$ & $e$ & $s_{i}$ & $s_{j}$ & $s_{i} s_{j}$ & $\left(\cdot \mid \sum \chi_{i}\right)$ & $\left(\cdot \mid \chi_{2}+\chi_{3}\right)$ & $\left(\cdot \mid \chi_{3}+\chi_{4}\right)$ & $\left(\cdot \mid \chi_{4}\right)$ \\
\hline$\rho_{111} \downarrow$ & 1 & 1 & 1 & 1 & 1 & 0 & 0 & 0 \\
$\left(\rho_{112}-\rho_{111}\right) \downarrow$ & 0 & 0 & 0 & 0 & 0 & 0 & 0 & 0 \\
$\left(\rho_{121}-\rho_{111}\right) \downarrow$ & 0 & 0 & -2 & -2 & 0 & 1 & 0 & 0 \\
$\left(\rho_{122}-\rho_{121}\right) \downarrow$ & 0 & 0 & 0 & 0 & 0 & 0 & 0 & 0 \\
$\left(\rho_{211}-\rho_{111}\right) \downarrow$ & 0 & -2 & 0 & -2 & 0 & 0 & 1 & 1 \\
$\left(\rho_{212}-\rho_{211}\right) \downarrow$ & 0 & 0 & 0 & 0 & 0 & 0 & 0 & 0 \\
$\left(\rho_{221}-\rho_{121}\right) \downarrow$ & 0 & -2 & 0 & 2 & 0 & 0 & 1 & 0 \\
$\left(\rho_{222}-\rho_{221}\right) \downarrow$ & 0 & 0 & 0 & 0 & 0 & 0 & 0 & 0
\end{tabular}

TABLE 23. Restricted characters and induced map $D_{2} \cong$ $\left\langle s_{i}, s_{j}\right\rangle \hookrightarrow \Delta(2,2,2)=C_{2} \times C_{2} \times C_{2}$.

column of Table 11 for $r$ equals $0,0, n-1$ and $n-1$ respectively, giving the restrictions (by abuse of notation we indicate the restricted character with the same 
symbols):

\begin{tabular}{c|cr|rr}
$C_{2} \times D_{m} \downarrow$ & $e$ & $s_{i}$ & $s_{j}$ & $s_{i} s_{j}$ \\
\hline$\rho_{1} \otimes \chi_{1}$ & 1 & 1 & 1 & 1 \\
$\rho_{1} \otimes \widehat{\chi_{2}}$ & 1 & 1 & -1 & -1 \\
$\rho_{1} \otimes \widehat{\chi_{3}}$ & 1 & 1 & -1 & -1 \\
$\rho_{1} \otimes \widehat{\chi_{4}}$ & 1 & 1 & 1 & 1 \\
$\rho_{1} \otimes \phi_{p}$ & 2 & 2 & 0 & 0 \\
\hline$\rho_{2} \otimes \chi_{1}$ & 1 & -1 & 1 & -1 \\
$\rho_{2} \otimes \chi_{2}$ & 1 & -1 & -1 & 1 \\
$\rho_{2} \otimes \widehat{\chi_{3}}$ & 1 & -1 & -1 & 1 \\
$\rho_{2} \otimes \widehat{\chi_{4}}$ & 1 & -1 & 1 & -1 \\
$\rho_{2} \otimes \phi_{p}$ & 2 & -2 & 0 & 0
\end{tabular}

Now we use Frobenius reciprocity (multiply the rows of the character tables of $H$ with those of $G \downarrow H$ above, and divide by $|H|=4$ ) to obtain the coefficients of the induced irreducible representations of $H$ in $G$ in terms of the irreducible representations of $G$. If $i<j$ then the character table of $H$ is the one given in Table 5 , but if $j<i$ we should interchange the second and third characters (rows) in that table. This gives

$$
\begin{aligned}
& \rho_{1} \otimes \rho_{1} \uparrow=\rho_{1} \otimes \chi_{1}+\widehat{\rho_{1} \otimes \chi_{4}}+\sum \rho_{1} \otimes \phi_{p} \\
& \rho_{1} \otimes \rho_{2} \uparrow=\rho_{1} \otimes \chi_{2}+\widehat{\rho_{1} \otimes \chi_{3}}+\sum \rho_{1} \otimes \phi_{p} \\
& \rho_{2} \otimes \rho_{1} \uparrow=\rho_{2} \otimes \chi_{1}+\widehat{\rho_{2} \otimes \chi_{4}}+\sum \rho_{2} \otimes \phi_{p} \\
& \rho_{2} \otimes \rho_{2} \uparrow=\rho_{2} \otimes \chi_{2}+\widehat{\rho_{2} \otimes \chi_{3}}+\sum \rho_{2} \otimes \phi_{p}
\end{aligned}
$$

if $i<j$, and

$$
\begin{aligned}
& \rho_{1} \otimes \rho_{1} \uparrow=\rho_{1} \otimes \chi_{1}+\widehat{\rho_{1} \otimes \chi_{4}}+\sum \rho_{1} \otimes \phi_{p} \\
& \rho_{1} \otimes \rho_{2} \uparrow=\rho_{2} \otimes \chi_{1}+\widehat{\rho_{2} \otimes \chi_{4}}+\sum \rho_{2} \otimes \phi_{p} \\
& \rho_{2} \otimes \rho_{1} \uparrow=\rho_{1} \otimes \chi_{2}+\widehat{\rho_{1} \otimes \chi_{3}}+\sum \rho_{1} \otimes \phi_{p} \\
& \rho_{2} \otimes \rho_{2} \uparrow=\rho_{2} \otimes \chi_{2}+\widehat{\rho_{2} \otimes \chi_{3}}+\sum \rho_{2} \otimes \phi_{p}
\end{aligned}
$$

if $j<i$. Equivalently, as a map of free abelian groups, we have the homomorphisms shown in Figure 5.

$$
\begin{aligned}
R_{\mathbb{C}}(H) & \rightarrow R_{\mathbb{C}}(G) \\
\mathbb{Z}^{4} & \rightarrow \mathbb{Z}^{2 \cdot c\left(D_{m}\right)} \\
(a, b, c, d) & \mapsto(a, b, \widehat{b}, \widehat{a}, a+b, \ldots, a+b, c, d, \widehat{d}, \widehat{c}, c+d, \ldots, c+d) \text { if } i<j, \\
(a, b, c, d) & \mapsto(a, c, \widehat{c}, \widehat{a}, a+c, \ldots, a+c, b, d, \widehat{d}, \widehat{b}, b+d, \ldots, b+d) \text { if } j<i
\end{aligned}
$$

Figure 5. Induction homomorphisms from $H=\left\langle s_{i}, s_{j}\right\rangle \cong C_{2} \times C_{2}$ to $G=\left\langle s_{i}, s_{j}, s_{k}\right\rangle \cong \Delta(2,2, m)=C_{2} \times D_{m}, m=m_{j k}>2$, and $j<k$.

Case 2: $H=\left\langle s_{i}, s_{k}\right\rangle \cong C_{2} \times C_{2}$. 
Restricting Table 11 to $H$ (1st, 3rd, 2nd and 4th column for $k$ equals 0 ) we get

\begin{tabular}{c|rr|rr}
$C_{2} \times D_{m} \downarrow$ & $e$ & $s_{i}$ & $s_{k}$ & $s_{i} s_{k}$ \\
\hline$\rho_{1} \otimes \chi_{1}$ & 1 & 1 & 1 & 1 \\
$\rho_{1} \otimes \chi_{2}$ & 1 & 1 & -1 & -1 \\
$\rho_{1} \otimes \widehat{\chi_{3}}$ & 1 & 1 & 1 & 1 \\
$\rho_{1} \otimes \widehat{\chi_{4}}$ & 1 & 1 & -1 & -1 \\
$\rho_{1} \otimes \phi_{p}$ & 2 & 2 & 0 & 0 \\
\hline$\rho_{1} \otimes \chi_{1}$ & 1 & -1 & 1 & -1 \\
$\rho_{1} \otimes \widehat{\chi_{2}}$ & 1 & -1 & -1 & 1 \\
$\rho_{1} \otimes \widehat{\chi_{3}}$ & 1 & -1 & 1 & -1 \\
$\rho_{1} \otimes \widehat{\chi_{4}}$ & 1 & -1 & -1 & 1 \\
$\rho_{1} \otimes \phi_{p}$ & 2 & -2 & 0 & 0
\end{tabular}

Via Frobenius reciprocity we obtain, assumming first $i<k$,

$$
\begin{aligned}
& \rho_{1} \otimes_{-} \quad \rho_{2} \otimes_{-} \\
& \rho_{1} \otimes \rho_{1} \uparrow=\rho_{1} \otimes \chi_{1}+\widehat{\rho_{1} \otimes \chi_{3}}+\sum \rho_{1} \otimes \phi_{p} \\
& \rho_{1} \otimes \rho_{2} \uparrow=\rho_{1} \otimes \chi_{2}+\widehat{\rho_{1} \otimes \chi_{4}}+\sum \rho_{1} \otimes \phi_{p} \\
& \rho_{2} \otimes \rho_{1} \uparrow=\quad \rho_{2} \otimes \chi_{1}+\widehat{\rho_{2} \otimes \chi_{3}}+\sum \rho_{2} \otimes \phi_{p} \\
& \rho_{2} \otimes \rho_{2} \uparrow=\quad \rho_{2} \otimes \chi_{2}+\widehat{\rho}_{2} \otimes \chi_{4}+\sum \rho_{2} \otimes \phi_{p}
\end{aligned}
$$

If $k<i$, the calculation is the same but we must interchange again the 2 nd and 3rd generators. All in all, we have the homomorphisms shown in Figure 6 as maps between free abelian groups.

$$
\begin{aligned}
R_{\mathbb{C}}(H) & \rightarrow R_{\mathbb{C}}(G) \\
\mathbb{Z}^{4} & \rightarrow \mathbb{Z}^{2 \cdot c\left(D_{m}\right)} \\
(a, b, c, d) & \mapsto(a, b, \widehat{a}, \widehat{b}, a+b, \ldots, a+b, c, d, \widehat{c}, \widehat{d}, c+d, \ldots, c+d) \text { if } i<k \\
(a, b, c, d) & \mapsto(a, c, \widehat{a}, \widehat{c}, a+c, \ldots, a+c, b, d, \widehat{b}, \widehat{d}, b+d, \ldots, b+d) \text { if } k<i
\end{aligned}
$$

FiguRE 6. Induction homomorphisms from $H=\left\langle s_{i}, s_{k}\right\rangle \cong C_{2} \times C_{2}$ to $G=\left\langle s_{i}, s_{j}, s_{k}\right\rangle \cong \Delta(2,2, m)=C_{2} \times D_{m}, m=m_{j k}>2$, and $j<k$.

Case 3: $H=\left\langle s_{j}, s_{k}\right\rangle \cong D_{m}$

In this case the condition $j<k$ already holds. Restricting Table 11 to $H$ (the first 
two columns)

\begin{tabular}{c|c}
$C_{2} \times D_{m} \downarrow$ & $\left(s_{j} s_{k}\right)^{r} \quad s_{k}\left(s_{j} s_{k}\right)^{r}$ \\
\hline$\rho_{1} \otimes \chi_{1}$ & \\
$\rho_{1} \otimes \chi_{2}$ & \\
$\rho_{1} \otimes \widehat{\chi_{3}}$ & $T_{m}$ \\
$\rho_{1} \otimes \widehat{\chi_{4}}$ & \\
$\rho_{1} \otimes \phi_{p}$ & \\
\hline$\rho_{2} \otimes \chi_{1}$ & \\
$\rho_{2} \otimes \chi_{2}$ & \\
$\rho_{2} \otimes \widehat{\chi_{3}}$ & $T_{m}$ \\
$\rho_{2} \otimes \widehat{\chi_{4}}$ & \\
$\rho_{2} \otimes \phi_{p}$ &
\end{tabular}

where $T_{m}$ are the coefficients of the character table of $D_{m}$ as in Table 4 . This immediately gives

$$
\begin{aligned}
& \chi_{i} \uparrow=\rho_{1} \otimes \chi_{i}+\rho_{2} \otimes \chi_{i} \text { for all } i \text {, and } \\
& \phi_{p} \uparrow=\rho_{1} \otimes \phi_{i}+\rho_{2} \otimes \phi_{p} \quad \text { for all } p \text {. }
\end{aligned}
$$

Equivalently, as a map of free abelian groups, this induction homomorphism is the one given in Figure 7.

$$
\begin{aligned}
R_{\mathbb{C}}(H) & \rightarrow R_{\mathbb{C}}(G) \\
\mathbb{Z}^{c\left(D_{m}\right)} & \rightarrow \mathbb{Z}^{2 \cdot c\left(D_{m}\right)} \\
\left(a, b, \widehat{c}, \widehat{d}, r_{1}, \ldots, r_{N}\right) & \mapsto\left(a, b, \widehat{c}, \widehat{d}, r_{1}, \ldots, r_{N}, a, b, \widehat{c}, \widehat{d}, r_{1}, \ldots, r_{N}\right) .
\end{aligned}
$$

FiguRE 7. Induction homomorphisms from $H=\left\langle s_{j}, s_{k}\right\rangle \cong D_{m}$ to $G=\left\langle s_{i}, s_{j}, s_{k}\right\rangle \cong \Delta(2,2, m)=C_{2} \times D_{m}, m=m_{j k}>2$, and $j<k$.

Finally, we compute the induction homomorphisms with respect to the transformed basis (Tables 6, 7, 8, 12, 13, 14 in Appendix A), summarising the results in Tables 24 to 29 .

B.3.3. $G=\Delta(2,3,3)$. This group is isomorphic to the symmetric group $S_{4}$ with Coxeter presentation

$$
\Delta(2,3,3)=\left\langle s_{i}, s_{j}, s_{k} \mid s_{i}^{2}, s_{j}^{2}, s_{k}^{2},\left(s_{i} s_{j}\right)^{3},\left(s_{i} s_{k}\right)^{2},\left(s_{j} s_{k}\right)^{3}\right\rangle
$$

and we assume $i<k$. We have again three relevant Coxeter subgroups $H$.

Case 1: $H=\left\langle s_{i}, s_{j}\right\rangle \cong D_{3}$

The expanded character table for $D_{3}$ (from Table 4), assuming first $i<j$, is

\begin{tabular}{c|cccccc}
$D_{3}$ & $e$ & $s_{i}$ & $s_{j}$ & $s_{i} s_{j}$ & $s_{j} s_{i}$ & $s_{i} s_{j} s_{i}$ \\
\hline$\chi_{1}$ & 1 & 1 & 1 & 1 & 1 & 1 \\
$\chi_{2}$ & 1 & -1 & -1 & 1 & 1 & -1 \\
$\phi_{1}$ & 2 & 0 & 0 & -1 & -1 & 0
\end{tabular}

There are 3 conjugacy classes, $\{e\},\left\{s_{i}, s_{j}, s_{i} s_{j} s_{i}=s_{j} s_{i} s_{j}\right\}$ and $\left\{s_{i} s_{j}, s_{j} s_{i}\right\}$, which remain unchanged if we swap $s_{i}$ and $s_{j}$, hence if $j<i$ the table stays the same and we do not have to treat those two cases separately. The character table of $G$ 


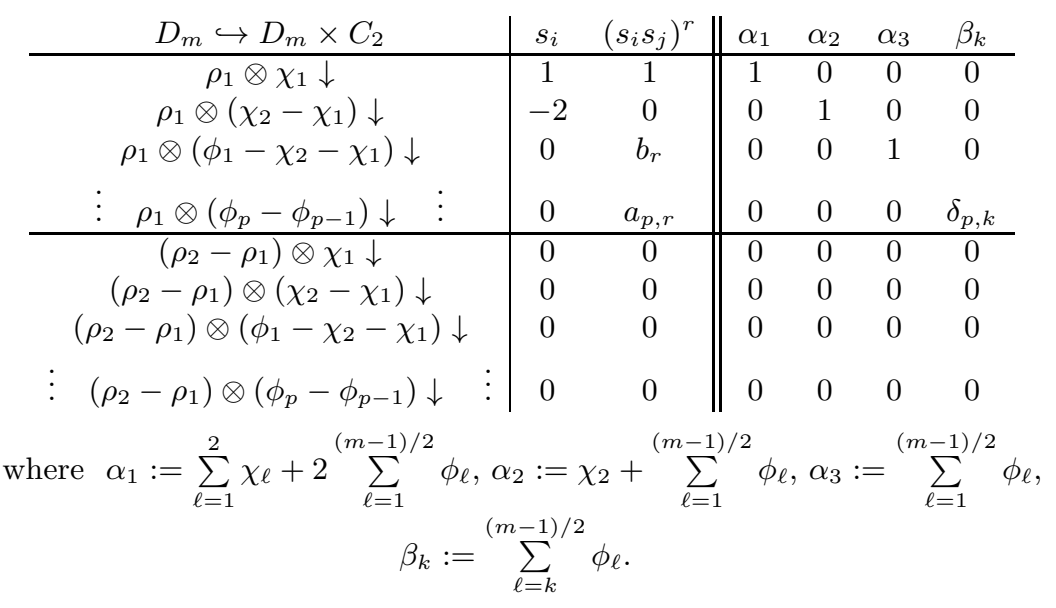

TABLE 24. Restricted characters and map induced by $D_{m} \hookrightarrow$ $\Delta(2,2, m)=D_{m} \times C_{2}$ for $m \geq 3$ odd. Here $a_{p, r}:=2 \cos \left(\frac{2 \pi p r}{m}\right)-$ $2 \cos \left(\frac{2 \pi(p-1) r}{m}\right), b_{r}:=2 \cos \left(\frac{2 \pi r}{m}\right)-2, \delta_{p, k}$ the Kronecker delta, $2 \leq p, k \leq \frac{m-1}{2}$ and $0 \leq r \leq \frac{m-1}{2}$.

\begin{tabular}{|c|c|c|c|c|c|c|c|c|}
\hline$D_{2} \hookrightarrow D_{m} \times C_{2}$ & $e$ & $s_{i}$ & $\alpha$ & $\alpha s_{i}$ & $\sum \chi_{i}$ & $\chi_{2}+\widehat{\chi_{3}}$ & $\widehat{\chi_{3}}$ & $\widehat{\chi_{3}}+\widehat{\chi_{4}}$ \\
\hline$\rho_{1} \otimes \chi_{1} \downarrow$ & 1 & 1 & 1 & 1 & 1 & 0 & 0 & 0 \\
\hline$\rho_{1} \otimes\left(\chi_{2}-\chi_{1}\right) \downarrow$ & 0 & -2 & 0 & -2 & 0 & 0 & 0 & 1 \\
\hline$\rho_{1} \otimes\left(\phi_{1}-\chi_{2}-\chi_{1}\right) \downarrow$ & 0 & 0 & 0 & 0 & 0 & 0 & 0 & 0 \\
\hline$\rho_{1} \otimes\left(\phi_{p}-\phi_{p-1}\right) \downarrow$ & 0 & 0 & 0 & 0 & 0 & 0 & 0 & 0 \\
\hline$\left(\rho_{2}-\rho_{1}\right) \otimes \chi_{1} \downarrow$ & 0 & 0 & $\overline{-2}$ & -2 & 0 & 1 & 0 & $\overline{0}$ \\
\hline$\left(\rho_{2}-\rho_{1}\right) \otimes\left(\chi_{2}-\chi_{1}\right) \downarrow$ & 0 & 0 & 0 & 4 & 0 & 0 & 1 & 0 \\
\hline$\left(\rho_{2}-\rho_{1}\right) \otimes\left(\phi_{1}-\chi_{2}-\chi_{1}\right) \downarrow$ & 0 & 0 & 0 & 0 & 0 & 0 & 0 & 0 \\
\hline$\left(\rho_{2}-\rho_{1}\right) \otimes\left(\phi_{p}-\phi_{p-1}\right) \downarrow$ & 0 & 0 & 0 & 0 & 0 & 0 & 0 & 0 \\
\hline
\end{tabular}

TABLE 25. Restricted characters and map induced by the two inclusions $D_{2} \hookrightarrow \Delta(2,2, m)=D_{m} \times C_{2}$, for $m \geq 3$ odd. Here $2 \leq p \leq \frac{m-1}{2}$.

(Table 15) restricted to $H$ consists on the 1st, 2nd, 2nd, 3rd, 3rd, 2nd columns (since $s_{i} \sim s_{j}, s_{j} s_{i} \sim s_{i} s_{j}$ and $s_{i} s_{j} s_{i} \sim s_{i}$ ):

\begin{tabular}{c|cccccc}
$S_{4} \downarrow$ & $e$ & $s_{i}$ & $s_{j}$ & $s_{i} s_{j}$ & $s_{j} s_{i}$ & $s_{i} s_{j} s_{i}$ \\
\hline$\xi_{1}$ & 1 & 1 & 1 & 1 & 1 & 1 \\
$\xi_{2}$ & 1 & -1 & -1 & 1 & 1 & -1 \\
$\xi_{3}$ & 2 & 0 & 0 & -1 & -1 & 0 \\
$\xi_{4}$ & 3 & 1 & 1 & 0 & 0 & 1 \\
$\xi_{5}$ & 3 & -1 & -1 & 0 & 0 & -1
\end{tabular}




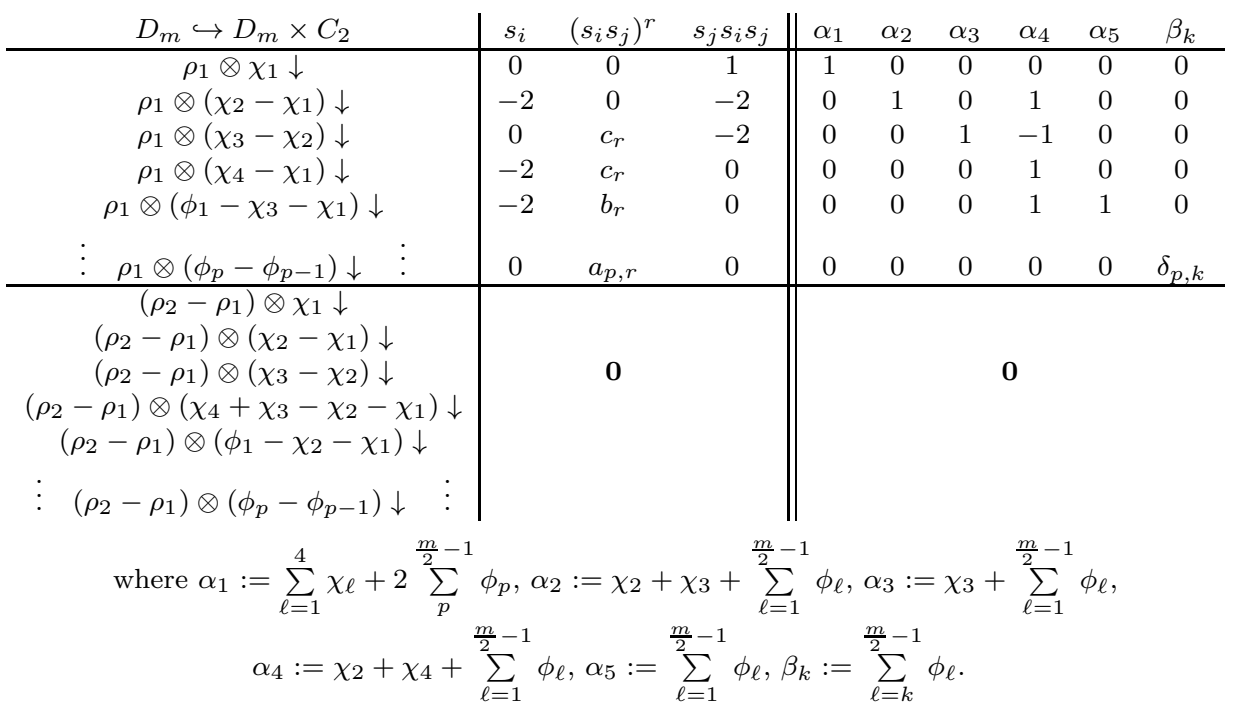

TABLE 26. Restricted characters and map induced by the inclusion $D_{m} \hookrightarrow \Delta(2,2, m)=D_{m} \times C_{2}$, for $m \geq 6$ even, not a power of 2 . Here $a_{p, r}:=2 \cos \left(\frac{2 \pi p r}{m}\right)-2 \cos \left(\frac{2 \pi(p-1) r}{m}\right), b_{r}:=2 \cos \left(\frac{2 \pi r}{m}\right)-(-1)^{r}-$ $1, c_{r}:=(-1)^{r}-1, \delta_{p, k}$ the Kronecker delta, $2 \leq p, k \leq \frac{m}{2}-1$, and $0 \leq r \leq \frac{m}{2}$.

\begin{tabular}{|c|c|c|c|c|c|c|c|c|}
\hline$D_{2} \hookrightarrow D_{m} \times C_{2}$ & $e$ & $s_{j} s_{i} s_{j}$ & $\alpha$ & $\alpha s_{j} s_{i} s_{j}$ & $\sum \chi_{i}$ & $\chi_{2}+\widehat{\chi_{3}}$ & $\widehat{\chi_{3}}$ & $\widehat{\chi_{3}}+\widehat{\chi_{4}}$ \\
\hline$\rho_{1} \otimes \chi_{1} \downarrow$ & 1 & 1 & 1 & 1 & 1 & 0 & 0 & 0 \\
\hline$\rho_{1} \otimes\left(\chi_{2}-\chi_{1}\right) \downarrow$ & 0 & -2 & 0 & -2 & 0 & 0 & 0 & 1 \\
\hline$\rho_{1} \otimes\left(\chi_{3}-\chi_{2}\right) \downarrow$ & 0 & 0 & 0 & 0 & 0 & 0 & 0 & 0 \\
\hline$\rho_{1} \otimes\left(\chi_{4}-\chi_{1}\right) \downarrow$ & 0 & 0 & 0 & 0 & 0 & 0 & 0 & 0 \\
\hline$\rho_{1} \otimes\left(\phi_{1}-\chi_{3}-\chi_{1}\right) \downarrow$ & 0 & 0 & 0 & 0 & 0 & 0 & 0 & 0 \\
\hline$\vdots \quad \rho_{1} \otimes\left(\phi_{p}-\phi_{p-1}\right) \downarrow \quad \vdots$ & 0 & 0 & 0 & 0 & 0 & 0 & 0 & 0 \\
\hline$\left(\rho_{2}-\rho_{1}\right) \otimes \chi_{1} \downarrow$ & 0 & 0 & -2 & -2 & 0 & 1 & 0 & 0 \\
\hline$\left(\rho_{2}-\rho_{1}\right) \otimes\left(\chi_{2}-\chi_{1}\right) \downarrow$ & 0 & 0 & 0 & 4 & 0 & 0 & 1 & 0 \\
\hline$\left(\rho_{2}-\rho_{1}\right) \otimes\left(\chi_{3}-\chi_{2}\right) \downarrow$ & 0 & 0 & 0 & 0 & 0 & 0 & 0 & 0 \\
\hline$\left(\rho_{2}-\rho_{1}\right) \otimes\left(\chi_{4}+\chi_{3}-\chi_{2}-\chi_{1}\right) \downarrow$ & 0 & 0 & 0 & 0 & 0 & 0 & 0 & 0 \\
\hline$\left(\rho_{2}-\rho_{1}\right) \otimes\left(\phi_{1}-\chi_{2}-\chi_{1}\right) \downarrow$ & 0 & 0 & 0 & 0 & 0 & 0 & 0 & 0 \\
\hline$\vdots \quad\left(\rho_{2}-\rho_{1}\right) \otimes\left(\phi_{p}-\phi_{p-1}\right) \downarrow$ & 0 & 0 & 0 & 0 & 0 & 0 & 0 & 0 \\
\hline
\end{tabular}

TABLE 27. Restricted characters and map induced by the two inclusions $D_{2} \hookrightarrow \Delta(2,2, m)=D_{m} \times C_{2}$, for $m \geq 2$ even, not a power of 2 . Here $2 \leq p \leq \frac{m}{2}-1$. Our choice on the generators is $(12)(34) \mapsto s_{j} s_{i} s_{j}$ and $(12) \mapsto \alpha$; any other choice yields an equivalent matrix.

Multiplying rows and dividing by $|H|=6$ we obtain

$$
\begin{aligned}
\chi_{1} \uparrow & =\xi_{1}+\xi_{4} \\
\chi_{2} \uparrow & =\xi_{2}+\xi_{5} \\
\phi_{1} \uparrow & =\xi_{3}+\xi_{4}+\xi_{5}
\end{aligned}
$$




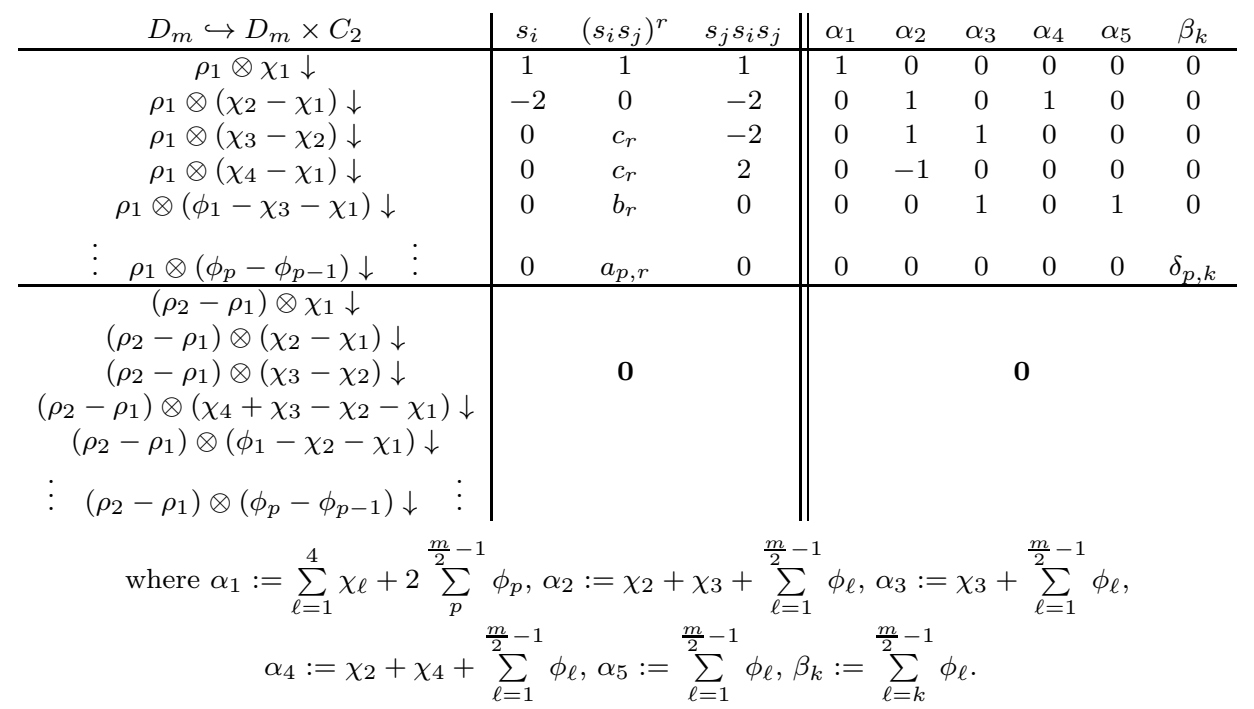

TABLE 28. Restricted characters and map induced by the inclusion $D_{m} \hookrightarrow \Delta(2,2, m)=D_{m} \times C_{2}$, for $m \geq 4$ a power of 2 . Here $a_{p, r}:=$ $2 \cos \left(\frac{2 \pi p r}{m}\right)-2 \cos \left(\frac{2 \pi(p-1) r}{m}\right), b_{r}:=2 \cos \left(\frac{2 \pi r}{m}\right)-2, c_{r}:=(-1)^{r}-1$, $\delta_{p, k}$ the Kronecker delta, $2 \leq p, k \leq \frac{m}{2}-1$ where $1<r<\frac{m}{2}$ and $1<k<\frac{m}{2}-1$.

\begin{tabular}{|c|c|c|c|c|c|c|c|c|}
\hline$D_{2} \hookrightarrow D_{m} \times C_{2}$ & $e$ & $s_{i}$ & $\alpha$ & $\alpha s_{i}$ & $\sum \chi_{i}$ & $\chi_{2}+\widehat{\chi_{3}}$ & $\widehat{\chi_{3}}$ & $\widehat{\chi_{3}}+\widehat{\chi_{4}}$ \\
\hline$\rho_{1} \otimes \chi_{1} \downarrow$ & 1 & 1 & 1 & 1 & 1 & 0 & 0 & 0 \\
\hline$\rho_{1} \otimes\left(\chi_{2}-\chi_{1}\right) \downarrow$ & 0 & -2 & 0 & -2 & 0 & 0 & 0 & 1 \\
\hline$\rho_{1} \otimes\left(\chi_{3}-\chi_{1}\right) \downarrow$ & 0 & 0 & 0 & 0 & 0 & 0 & 0 & 0 \\
\hline$\rho_{1} \otimes\left(\chi_{4}-\chi_{2}\right) \downarrow$ & 0 & 0 & 0 & 0 & 0 & 0 & 0 & 0 \\
\hline$\rho_{1} \otimes\left(\phi_{1}-\chi_{2}-\chi_{1}\right) \downarrow$ & 0 & 0 & 0 & 0 & 0 & 0 & 0 & 0 \\
\hline$\vdots \quad \rho_{1} \otimes\left(\phi_{p}-\phi_{p-1}\right) \downarrow \quad \vdots$ & 0 & 0 & 0 & 0 & 0 & 0 & 0 & 0 \\
\hline$\left(\rho_{2}-\rho_{1}\right) \otimes \chi_{1} \downarrow$ & 0 & 0 & -2 & -2 & 0 & 1 & 0 & 0 \\
\hline$\left(\rho_{2}-\rho_{1}\right) \otimes\left(\chi_{2}-\chi_{1}\right) \downarrow$ & 0 & 0 & 0 & 4 & 0 & 0 & 1 & 0 \\
\hline$\left(\rho_{2}-\rho_{1}\right) \otimes\left(\chi_{3}-\chi_{1}\right) \downarrow$ & 0 & 0 & 0 & 0 & 0 & 0 & 0 & 0 \\
\hline$\left(\rho_{2}-\rho_{1}\right) \otimes\left(\chi_{4}+\chi_{3}-\chi_{2}-\chi_{1}\right) \downarrow$ & 0 & 0 & 0 & 0 & 0 & 0 & 0 & 0 \\
\hline$\left(\rho_{2}-\rho_{1}\right) \otimes\left(\phi_{1}-\chi_{2}-\chi_{1}\right) \downarrow$ & 0 & 0 & 0 & 0 & 0 & 0 & 0 & 0 \\
\hline$\vdots \quad\left(\rho_{2}-\rho_{1}\right) \otimes\left(\phi_{p}-\phi_{p-1}\right) \downarrow$ & 0 & 0 & 0 & 0 & 0 & 0 & 0 & 0 \\
\hline
\end{tabular}

TABLE 29. Restricted characters and map induced by the two inclusions $D_{2} \hookrightarrow \Delta(2,2, m)=D_{m} \times C_{2}$, for $m \geq 4$ a power of 2 . Here $2 \leq p \leq \frac{m}{2}-1$. Our choice on the generators is $(12)(34) \mapsto s_{i}$ and (12) $\mapsto \alpha$, and any other choice yields an equivalent matrix.

Equivalently, we have the map of free abelian groups shown in Figure 8.

$\underline{\text { Case } 2: H=\left\langle s_{j}, s_{k}\right\rangle \cong D_{3}}$ 


$$
\begin{aligned}
R_{\mathbb{C}}(H) & \rightarrow R_{\mathbb{C}}(G) \\
\mathbb{Z}^{3} & \rightarrow \mathbb{Z}^{5} \\
(a, b, c) & \mapsto(a, b, c, a+c, b+c) .
\end{aligned}
$$

FiguRE 8. Induction homomorphism from $H=\left\langle s_{i}, s_{j}\right\rangle \cong D_{3}$ or $H=\left\langle s_{j}, s_{k}\right\rangle \cong D_{3}$ to $G=\left\langle s_{i}, s_{j}, s_{k}\right\rangle \cong \Delta(2,3,3)=S_{4}$, and $i<k$.

This case is completely analogous to the previous one, so we obtain the same map, also shown in Figure 8.

Case 3: $H=\left\langle s_{i}, s_{k}\right\rangle \cong D_{2}=C_{2} \times C_{2}$

The character table of $G$ (Table 15 ) restricted to $H$ consists on the 1 st, 2 nd, 2 nd, 5th columns:

\begin{tabular}{c|cccc}
$S_{4} \downarrow$ & $e$ & $s_{i}$ & $s_{k}$ & $s_{i} s_{k}$ \\
\hline$\xi_{1}$ & 1 & 1 & 1 & 1 \\
$\xi_{2}$ & 1 & -1 & -1 & 1 \\
$\xi_{3}$ & 2 & 0 & 0 & 2 \\
$\xi_{4}$ & 3 & 1 & 1 & -1 \\
$\xi_{5}$ & 3 & -1 & -1 & -1
\end{tabular}

Multiplying the rows with the characters of $H$ (Table 5) and dividing by $|H|=4$ we obtain (note that $i<k$ already holds)

$$
\begin{aligned}
\rho_{1} \otimes \rho_{1} \uparrow & =\xi_{1}+\xi_{3}+\xi_{4} \\
\rho_{1} \otimes \rho_{2} \uparrow & =\xi_{4}+\xi_{5} \\
\rho_{2} \otimes \rho_{1} \uparrow & =\xi_{4}+\xi_{5} \\
\rho_{2} \otimes \rho_{2} \uparrow & =\xi_{2}+\xi_{3}+\xi_{5}
\end{aligned}
$$

Equivalently, this is the homomorphism of abelian groups shown in Figure 9. Note that this is the first time that of an induction homomorphism with nontrivial kernel.

$$
\begin{aligned}
R_{\mathbb{C}}(H) & \rightarrow R_{\mathbb{C}}(G) \\
\mathbb{Z}^{4} & \rightarrow \mathbb{Z}^{5} \\
(a, b, c, d) & \mapsto(a, d, a+d, a+b+c, b+c+d) .
\end{aligned}
$$

FiguRE 9. Induction homomorphism from $H=\left\langle s_{i}, s_{k}\right\rangle \cong C_{2} \times C_{2}$ to $G=\left\langle s_{i}, s_{j}, s_{k}\right\rangle \cong \Delta(2,3,3)=S_{4}$, and $i<k$.

Now we give the induction homomorphisms with respect to the transformed bases (Tables 6, 7, 16 in Appendix A), summarised in Table 30.

B.3.4. $G=\Delta(2,3,4)$. This group is isomorphic to $S_{4} \times C_{2}$ with Coxeter presentation

$$
\Delta(2,3,4)=\left\langle s_{i}, s_{j}, s_{k} \mid s_{i}^{2}, s_{j}^{2}, s_{k}^{2},\left(s_{i} s_{j}\right)^{3},\left(s_{i} s_{k}\right)^{2},\left(s_{j} s_{k}\right)^{4}\right\rangle .
$$

The three relevant induction homomorphism are as follows.

Case 1: $H=\left\langle s_{i}, s_{k}\right\rangle \cong D_{2}=C_{2} \times C_{2}$ 


\begin{tabular}{|c|c|c|c|c|c|c|c|c|}
\hline$D_{2} \hookrightarrow S_{4}$ & $(1)$ & $\left(\begin{array}{ll}1 & 2\end{array}\right)$ & & $(12)(34)$ & $\left(\widetilde{\xi}_{i} \mid \sum \chi_{i}\right) \quad\left(\widetilde{\xi}_{i}\right.$ & $\left.\widetilde{\xi}_{i} \mid \chi_{2}+\widehat{\chi_{3}}\right)$ & $\left(\widetilde{\xi}_{i} \mid \widehat{\chi_{3}}\right.$ & $\left(\widetilde{\xi_{i}} \mid \widehat{\chi_{4}}\right)$ \\
\hline$\widetilde{\xi_{1} \downarrow}$ & 1 & 1 & & 1 & 1 & 0 & 0 & 0 \\
\hline$\widetilde{\xi_{2}} \downarrow$ & 0 & -2 & & 0 & 0 & 1 & 0 & 0 \\
\hline$\widetilde{\xi_{3}} \downarrow$ & 0 & 0 & & 0 & 0 & 0 & 0 & 0 \\
\hline$\widetilde{\xi}_{4} \downarrow$ & 0 & 0 & & -4 & 0 & 0 & 1 & 1 \\
\hline$\widetilde{\xi}_{5} \downarrow$ & 0 & 0 & & 0 & 0 & 0 & 0 & 0 \\
\hline \multicolumn{2}{|c|}{$D_{3} \hookrightarrow S_{4}$} & (1) & $(12)$ & $(123)$ & $\left(\widetilde{\xi}_{i} \mid 2 \phi_{1}+\sum \chi_{i}\right)$ & \multicolumn{3}{|c|}{ i) $\quad\left(\widetilde{\xi}_{i} \mid \chi_{2}+\phi_{1}\right) \quad\left(\widetilde{\xi}_{i} \mid \phi_{1}\right)$} \\
\hline \multicolumn{2}{|c|}{$\widetilde{\xi_{1}} \downarrow$} & 1 & 1 & 1 & 1 & 0 & \multicolumn{2}{|r|}{0} \\
\hline \multicolumn{2}{|c|}{$\widetilde{\xi_{2}} \downarrow$} & 0 & -2 & 0 & 0 & 1 & \multicolumn{2}{|r|}{0} \\
\hline \multicolumn{2}{|c|}{$\underset{\widetilde{\xi_{3}}}{\widetilde{\sim}} \downarrow$} & 0 & 0 & -3 & 0 & 0 & \multicolumn{2}{|r|}{1} \\
\hline \multicolumn{2}{|c|}{$\widetilde{\xi}_{4} \downarrow$} & 0 & 0 & 0 & 0 & 0 & \multicolumn{2}{|r|}{0} \\
\hline \multicolumn{2}{|c|}{$\widetilde{\xi}_{5} \downarrow$} & 0 & 0 & 0 & 0 & 0 & \multicolumn{2}{|r|}{0} \\
\hline
\end{tabular}

TABLE 30. Restricted characters and map induced by the inclusions of $D_{2}$ and $D_{3}$ into $S_{4}$. The second inclusion $\langle(23),(34)\rangle \cong$ $D_{3} \hookrightarrow S_{4}$ induces the same map as the first one, because $(23) \sim$ (12) and (234) (123).

The character table of $G$ (Table 17) restricted to $H$ consists on the 1st, 7th, 10th and 2nd columns:

\begin{tabular}{c|cccc}
$S_{4} \times C_{2} \downarrow$ & $e$ & $s_{i}$ & $s_{k}$ & $s_{i} s_{k}$ \\
\hline$\rho_{1} \otimes \xi_{1}$ & 1 & 1 & 1 & 1 \\
$\rho_{1} \otimes \xi_{2}$ & 1 & -1 & 1 & -1 \\
$\rho_{1} \otimes \xi_{3}$ & 2 & 0 & 2 & 0 \\
$\rho_{1} \otimes \xi_{4}$ & 3 & 1 & -1 & 1 \\
$\rho_{1} \otimes \xi_{5}$ & 3 & -1 & -1 & -1 \\
\hline$\rho_{2} \otimes \xi_{1}$ & 1 & -1 & -1 & 1 \\
$\rho_{2} \otimes \xi_{2}$ & 1 & 1 & -1 & -1 \\
$\rho_{2} \otimes \xi_{3}$ & 2 & 0 & -2 & 0 \\
$\rho_{2} \otimes \xi_{4}$ & 3 & -1 & 1 & 1 \\
$\rho_{2} \otimes \xi_{5}$ & 3 & 1 & 1 & -1
\end{tabular}

Suppose first that $i<k$. Multiplying these rows with the rows of Table 5 we deduce that (note the shortcut in notation)

$$
\begin{array}{rll} 
& \rho_{1} \otimes{ }_{-} & \rho_{2} \otimes \\
\rho_{1} \otimes \rho_{1} \uparrow & =\xi_{1}+\xi_{3}+\xi_{4}+ & \xi_{4}+\xi_{5} \\
\rho_{1} \otimes \rho_{2} \uparrow & =\xi_{4}+\xi_{5}+ & \xi_{2}+\xi_{3}+\xi_{5} \\
\rho_{2} \otimes \rho_{1} \uparrow & =\xi_{2}+\xi_{3}+\xi_{5}+ & \xi_{4}+\xi_{5} \\
\rho_{2} \otimes \rho_{2} \uparrow & =\xi_{4}+\xi_{5}+ & \xi_{1}+\xi_{3}+\xi_{4}
\end{array}
$$

On the other hand, if $k<i$, then we should interchange the 2nd and 3rd generators. All in all, we have the homomorphisms of free abelian groups shown in Figure 10.

Case $2: H=\left\langle s_{i}, s_{j}\right\rangle \cong D_{3}$ 


$$
\begin{aligned}
R_{\mathbb{C}}(H) & \rightarrow R_{\mathbb{C}}(G) \\
\mathbb{Z}^{4} & \rightarrow \mathbb{Z}^{10} \\
(a, b, c, d) & \mapsto(a, c, a+c, a+b+d, b+c+d, d, b, b+d, a+c+d, a+b+c) \text { if } i<k, \\
(a, b, c, d) & \mapsto(a, b, a+b, a+c+d, b+c+d, d, c, c+d, a+b+d, a+b+c) \text { if } k<i .
\end{aligned}
$$

FIgURE 10. Induction homomorphism from $H=\left\langle s_{i}, s_{k}\right\rangle \cong C_{2} \times$ $C_{2}$ to $G=\left\langle s_{i}, s_{j}, s_{k}\right\rangle \cong \Delta(2,3,4)=S_{4} \times C_{2}$.

The characters of $G$ (Table 17 ) restricted to $H$ consists on the 1 st, 7 th, 7 th, $3 \mathrm{rd}$, 3rd, 7th columns:

\begin{tabular}{c|cccccc}
$S_{4} \times C_{2} \downarrow$ & $e$ & $s_{i}$ & $s_{j}$ & $s_{i} s_{j}$ & $s_{j} s_{i}$ & $s_{i} s_{j} s_{i}$ \\
\hline$\rho_{1} \otimes \xi_{1}$ & 1 & 1 & 1 & 1 & 1 & 1 \\
$\rho_{1} \otimes \xi_{2}$ & 1 & -1 & -1 & 1 & 1 & -1 \\
$\rho_{1} \otimes \xi_{3}$ & 2 & 0 & 0 & -1 & -1 & 0 \\
$\rho_{1} \otimes \xi_{4}$ & 3 & 1 & 1 & 0 & 0 & 1 \\
$\rho_{1} \otimes \xi_{5}$ & 3 & -1 & -1 & 0 & 0 & -1 \\
\hline$\rho_{2} \otimes \xi_{1}$ & 1 & -1 & -1 & 1 & 1 & -1 \\
$\rho_{2} \otimes \xi_{2}$ & 1 & 1 & 1 & 1 & 1 & 1 \\
$\rho_{2} \otimes \xi_{3}$ & 2 & 0 & 0 & -1 & -1 & 0 \\
$\rho_{2} \otimes \xi_{4}$ & 3 & -1 & -1 & 0 & 0 & -1 \\
$\rho_{2} \otimes \xi_{5}$ & 3 & 1 & 1 & 0 & 0 & 1
\end{tabular}

Multiplying these rows with the rows of character table of $D_{3}$ above we deduce that (recall that this table is fixed by interchanging the Coxeter generators)

$$
\begin{array}{rll} 
& \rho_{1} \otimes{ }_{-} & \rho_{2} \otimes- \\
\chi_{1} \uparrow=\xi_{1}+\xi_{4}+ & \xi_{2}+\xi_{5} \\
\chi_{1} \uparrow=\xi_{2}+\xi_{5}+ & \xi_{1}+\xi_{4} \\
\phi_{1} \uparrow= & \xi_{3}+\xi_{4}+\xi_{5}+ & \xi_{3}+\xi_{4}+\xi_{5}
\end{array}
$$

or, equivalently, the linear map shown in Figure 11.

$$
\begin{aligned}
R_{\mathbb{C}}(H) & \rightarrow R_{\mathbb{C}}(G) \\
\mathbb{Z}^{3} & \rightarrow \mathbb{Z}^{10} \\
(a, b, c) & \mapsto(a, b, c, a+c, b+c, b, a, c, b+c, a+c) .
\end{aligned}
$$

FiguRE 11. Induction homomorphism from $H=\left\langle s_{i}, s_{j}\right\rangle \cong D_{3}$ to $G=\left\langle s_{i}, s_{j}, s_{k}\right\rangle \cong \Delta(2,3,4)=S_{4} \times C_{2}$.

Case 3: $H=\left\langle s_{j}, s_{k}\right\rangle \cong D_{4}$ 
First we expand the character table of $D_{4}$, assuming $j<k$,

\begin{tabular}{c|cccccccc}
$D_{4}$ & $e$ & $s_{j}$ & $s_{k}$ & $s_{j} s_{k}$ & $s_{k} s_{j}$ & $s_{j} s_{k} s_{j}$ & $s_{k} s_{j} s_{k}$ & $s_{j} s_{k} s_{j} s_{k}$ \\
\hline$\chi_{1}$ & 1 & 1 & 1 & 1 & 1 & 1 & 1 & 1 \\
$\chi_{2}$ & 1 & -1 & -1 & 1 & 1 & -1 & -1 & 1 \\
$\chi_{3}$ & 1 & -1 & 1 & -1 & -1 & 1 & -1 & 1 \\
$\chi_{4}$ & 1 & 1 & -1 & -1 & -1 & -1 & 1 & 1 \\
$\phi_{1}$ & 2 & 0 & 0 & 0 & 0 & 0 & 0 & -2
\end{tabular}

Note that if $k<j$ we should interchange the characters $\chi_{3}$ and $\chi_{4}$ in order to maintain the notation consistent. The characters of $G$ restricted to $H$ are the 1st, 7th, 10th, 4th, 4th, 10th, 7th, 5th columns of Table 17:

\begin{tabular}{c|cccccccc}
$S_{4} \times C_{2} \downarrow$ & $e$ & $s_{2}$ & $s_{3}$ & $s_{2} s_{3}$ & $s_{3} s_{2}$ & $s_{2} s_{3} s_{2}$ & $s_{3} s_{2} s_{3}$ & $s_{2} s_{3} s_{2} s_{3}$ \\
\hline$\rho_{1} \otimes \xi_{1}$ & 1 & 1 & 1 & 1 & 1 & 1 & 1 & 1 \\
$\rho_{1} \otimes \xi_{2}$ & 1 & -1 & 1 & -1 & -1 & 1 & -1 & 1 \\
$\rho_{1} \otimes \xi_{3}$ & 2 & 0 & 2 & 0 & 0 & 2 & 0 & 2 \\
$\rho_{1} \otimes \xi_{4}$ & 3 & 1 & -1 & -1 & -1 & -1 & 1 & -1 \\
$\rho_{1} \otimes \xi_{5}$ & 3 & -1 & -1 & 1 & 1 & -1 & -1 & -1 \\
\hline$\rho_{2} \otimes \xi_{1}$ & 1 & -1 & -1 & 1 & 1 & -1 & -1 & 1 \\
$\rho_{2} \otimes \xi_{2}$ & 1 & 1 & -1 & -1 & -1 & -1 & 1 & 1 \\
$\rho_{2} \otimes \xi_{3}$ & 2 & 0 & -2 & 0 & 0 & -2 & 0 & 2 \\
$\rho_{2} \otimes \xi_{4}$ & 3 & -1 & 1 & -1 & -1 & 1 & -1 & -1 \\
$\rho_{2} \otimes \xi_{5}$ & 3 & 1 & 1 & 1 & 1 & 1 & 1 & -1
\end{tabular}

Multiplying these rows with the rows of the character table of $D_{4}$ above we deduce that

$$
\begin{array}{rll} 
& \rho_{1} \otimes_{-} & \rho_{2} \otimes_{-} \\
\chi_{1} \uparrow & =\xi_{1}+\xi_{3}+ & \xi_{5} \\
\chi_{2} \uparrow & =\xi_{5}+ & \xi_{1}+\xi_{3} \\
\chi_{3} \uparrow & =\xi_{2}+\xi_{3}+ & \xi_{4} \\
\chi_{4} \uparrow & =\xi_{4}+ & \xi_{2}+\xi_{3} \\
\phi_{1} \uparrow & =\xi_{4}+\xi_{5}+ & \xi_{4}+\xi_{5}
\end{array}
$$

The computation in the case $k<j$ is identical, but interchanging $\chi_{3}$ and $\chi_{4}$. All in all, we have the induction homomorphisms shown, as maps between abelian groups, in Figure $\mathrm{X}$

$$
\begin{aligned}
R_{\mathbb{C}}(H) & \rightarrow R_{\mathbb{C}}(G) \\
\mathbb{Z}^{5} & \rightarrow \mathbb{Z}^{10} \\
(a, b, c, d, e) & \mapsto(a, c, a+c, d+e, b+e, b, d, b+d, c+e, a+e) \text { if } j<k \\
(a, b, c, d, e) & \mapsto(a, d, a+d, c+e, b+e, b, c, b+c, d+e, a+e) \text { if } k<j
\end{aligned}
$$

FIGURE 12. Induction homomorphism from $H=\left\langle s_{j}, s_{k}\right\rangle \cong D_{4}$ to $G=\left\langle s_{i}, s_{j}, s_{k}\right\rangle \cong \Delta(2,3,4)=S_{4} \times C_{2}$.

On the other hand, the induction homomorphisms with respect to the transformed bases (Tables 7, 8, 18 in Appendix A) are summarised in Tables 31, 32 and 33. 


\begin{tabular}{c|cccc||cccc}
$D_{2} \hookrightarrow S_{4} \times C_{2}$ & $(1)$ & $\alpha(12)$ & $\alpha(12)(34)$ & $(34)$ & $\left(\cdot \mid \sum \chi_{i}\right)$ & $\left(\cdot \mid \chi_{2}+\widehat{\chi_{3}}\right)$ & $\left(\cdot \mid \widehat{\chi_{3}}+\widehat{\chi_{4}}\right)$ & $\left(\cdot \mid \widehat{\chi_{4}}\right)$ \\
\hline$\alpha_{1} \downarrow$ & 1 & 1 & 1 & 1 & 1 & 0 & 0 & 0 \\
$\alpha_{2} \downarrow$ & 0 & -2 & 0 & -2 & 0 & 1 & 0 & 0 \\
$\alpha_{3} \downarrow$ & 0 & 0 & 0 & 0 & 0 & 0 & 0 & 0 \\
$\alpha_{4} \downarrow$ & 0 & 0 & -2 & -2 & 0 & 0 & 1 & 1 \\
$\alpha_{5} \downarrow$ & 0 & 0 & 0 & 0 & 0 & 0 & 0 & 0 \\
\hline$\alpha_{6} \downarrow$ & 0 & 0 & -2 & 2 & 0 & 0 & 1 & 0 \\
$\alpha_{7} \downarrow$ & 0 & 0 & 0 & -4 & 0 & 0 & 0 & 1 \\
$\alpha_{8} \downarrow$ & 0 & 0 & 0 & 0 & 0 & 0 & 0 & 0 \\
$\alpha_{9} \downarrow$ & 0 & 0 & 0 & 0 & 0 & 0 & 0 & 0 \\
$\alpha_{10} \downarrow$ & 0 & 0 & 0 & 0 & 0 & 0 & 0 & 0
\end{tabular}

TABLE 31. Restricted characters and map induced by the inclusion of $D_{2}$ into $S_{4} \times C_{2}$. Here $\alpha_{1}, \ldots, \alpha_{10}$ are as in Table 18 .

\begin{tabular}{c|ccccc}
$D_{4} \hookrightarrow S_{4} \times C_{2}$ & $(1)$ & $\alpha(13)$ & $(13)(24)$ & $\alpha(12)(34)$ & $(1432)$ \\
\hline$\alpha_{1} \downarrow$ & 1 & 1 & 1 & 1 & 1 \\
$\alpha_{2} \downarrow$ & 0 & -2 & 0 & 0 & -2 \\
$\alpha_{3} \downarrow$ & 0 & 0 & 0 & 0 & 0 \\
$\alpha_{4} \downarrow$ & 0 & 0 & -4 & -2 & 4 \\
$\alpha_{5} \downarrow$ & 0 & 0 & 0 & 0 & 4 \\
\hline$\alpha_{6} \downarrow$ & 0 & 0 & 0 & -2 & 2 \\
$\alpha_{7} \downarrow$ & 0 & 0 & 0 & 0 & 0 \\
$\alpha_{8} \downarrow$ & 0 & 0 & 0 & 0 & 0 \\
$\alpha_{9} \downarrow$ & 0 & 0 & 0 & 0 & 0 \\
$\alpha_{10} \downarrow$ & 0 & 0 & 0 & 0 & 0
\end{tabular}

\begin{tabular}{c|ccccc}
$D_{4} \hookrightarrow S_{4} \times C_{2}$ & $\left(\cdot \mid 2 \phi_{1}+\sum \chi_{i}\right)$ & $\left(\cdot \mid \chi_{2}+\widehat{\chi_{3}}+\phi_{1}\right)$ & $\left(\cdot \mid \widehat{\chi_{3}}+\phi_{1}\right)$ & $\left(\cdot \mid \widehat{\chi_{4}}+\chi_{2}\right)$ & $\left(\cdot \mid \phi_{1}\right)$ \\
\hline$\alpha_{1} \downarrow$ & 1 & 0 & 0 & 0 & 0 \\
$\alpha_{2} \downarrow$ & 0 & 0 & 0 & 1 & 0 \\
$\alpha_{3} \downarrow$ & 0 & 0 & 0 & 0 & 0 \\
$\alpha_{4} \downarrow$ & 0 & 1 & 0 & -1 & 1 \\
$\alpha_{5} \downarrow$ & 0 & 0 & -1 & 0 & 0 \\
\hline$\alpha_{6} \downarrow$ & 0 & 1 & 0 & 0 & 0 \\
$\alpha_{7} \downarrow$ & 0 & 0 & 0 & 0 & 0 \\
$\alpha_{8} \downarrow$ & 0 & 0 & 0 & 0 & 0 \\
$\alpha_{9} \downarrow$ & 0 & 0 & 0 & 0 & 0 \\
$\alpha_{10} \downarrow$ & 0 & 0 & 0 & 0 & 0
\end{tabular}

TABLE 32. Restricted characters (top) and map induced by the inclusion of $D_{4}$ into $S_{4} \times C_{2}$ (bottom). Here $\alpha_{1}, \ldots, \alpha_{10}$ are as in Table 18.

B.3.5. $G=\Delta(2,3,5)$. This group is isomorphic to $A_{5} \times C_{2}$ with Coxeter presentation

$$
\Delta(2,3,5)=\left\langle s_{i}, s_{j}, s_{k} \mid s_{i}^{2}, s_{j}^{2}, s_{k}^{2},\left(s_{i} s_{j}\right)^{3},\left(s_{i} s_{k}\right)^{2},\left(s_{j} s_{k}\right)^{5}\right\rangle .
$$

We have again three relevant induction homomorphisms.

Case 1: $H=\left\langle s_{i}, s_{k}\right\rangle \cong D_{2}=C_{2} \times C_{2}$

The characters of $G$ (Table 19) restricted to $H$ consists on the 1st, 8th, 8th, 3rd 


\begin{tabular}{c|ccc||ccc}
$D_{3} \hookrightarrow S_{4} \times C_{2}$ & $(1)$ & $\alpha(12)$ & $(123)$ & $\left(\cdot \mid 2 \phi_{1}+\sum \chi_{i}\right)$ & $\left(\cdot \mid \chi_{2}+\phi_{1}\right)$ & $\left(\cdot \mid \phi_{1}\right)$ \\
\hline$\alpha_{1} \downarrow$ & 1 & 1 & 1 & 1 & 0 & 0 \\
$\alpha_{2} \downarrow$ & 0 & -2 & 0 & 0 & 1 & 0 \\
$\alpha_{3} \downarrow$ & 0 & 0 & -3 & 0 & 0 & 1 \\
$\alpha_{4} \downarrow$ & 0 & 0 & 0 & 0 & 0 & 0 \\
$\alpha_{5} \downarrow$ & 0 & 0 & 0 & 0 & 0 & 0 \\
\hline$\alpha_{6} \downarrow$ & 0 & 0 & 0 & 0 & 0 & 0 \\
$\alpha_{7} \downarrow$ & 0 & 0 & 0 & 0 & 0 & 0 \\
$\alpha_{8} \downarrow$ & 0 & 0 & 0 & 0 & 0 & 0 \\
$\alpha_{9} \downarrow$ & 0 & 0 & 0 & 0 & 0 & 0 \\
$\alpha_{10} \downarrow$ & 0 & 0 & 0 & 0 & 0 & 0
\end{tabular}

TABLE 33. Restricted characters and map induced by the inclusion of $D_{3}$ into $S_{4} \times C_{2}$. Here $\alpha_{1}, \ldots, \alpha_{10}$ are as in Table 18 .

columns

\begin{tabular}{c|cccc}
$A_{5} \times C_{2} \downarrow$ & $e$ & $s_{i}$ & $s_{k}$ & $s_{i} s_{k}$ \\
\hline$\rho_{1} \otimes \xi_{1}$ & 1 & 1 & 1 & 1 \\
$\rho_{1} \otimes \xi_{2}$ & 4 & 0 & 0 & 0 \\
$\rho_{1} \otimes \xi_{3}$ & 5 & 1 & 1 & 1 \\
$\rho_{1} \otimes \xi_{4}$ & 3 & -1 & -1 & -1 \\
$\rho_{1} \otimes \xi_{5}$ & 3 & -1 & -1 & -1 \\
\hline$\rho_{2} \otimes \xi_{1}$ & 1 & -1 & -1 & 1 \\
$\rho_{2} \otimes \xi_{2}$ & 4 & 0 & 0 & 0 \\
$\rho_{2} \otimes \xi_{3}$ & 5 & -1 & -1 & 1 \\
$\rho_{2} \otimes \xi_{4}$ & 3 & 1 & 1 & -1 \\
$\rho_{2} \otimes \xi_{5}$ & 3 & 1 & 1 & -1
\end{tabular}

Suppose first $i<k$. Multiplying these rows with the rows of Table 5 we obtain

$$
\begin{array}{lll} 
& \rho_{1} \otimes_{-} & \rho_{2} \otimes \\
\rho_{1} \otimes \rho_{1} \uparrow & =\xi_{1}+\xi_{2}+2 \xi_{3}+ & \xi_{2}+\xi_{3}+\xi_{4}+\xi_{5} \\
\rho_{1} \otimes \rho_{2} \uparrow= & \xi_{2}+\xi_{3}+\xi_{4}+\xi_{5}+ & \xi_{2}+\xi_{3}+\xi_{4}+\xi_{5} \\
\rho_{2} \otimes \rho_{1} \uparrow= & \xi_{2}+\xi_{3}+\xi_{4}+\xi_{5}+ & \xi_{2}+\xi_{3}+\xi_{4}+\xi_{5} \\
\rho_{2} \otimes \rho_{2} \uparrow= & \xi_{2}+\xi_{3}+\xi_{4}+\xi_{5}+ & \xi_{1}+\xi_{2}+2 \xi_{3}
\end{array}
$$

If $k<i$ we must interchange the 2 nd and 3 rd generators, but note that we obtain the same map. All in one, we have one induction map, given as a homomorphism of free abelian groups in Figure 13.

$$
\begin{aligned}
R_{\mathbb{C}}(H) & \rightarrow R_{\mathbb{C}}(G) \\
\mathbb{Z}^{4} \rightarrow \mathbb{Z}^{10} & \\
(a, b, c, d) \rightarrow & (a, b+c+d, 2 a+b+c+d, b+c+d, b+c+d, \\
& d, a+b+c, a+b+c+2 d, a+b+c, a+b+c) .
\end{aligned}
$$

FIGURE 13. Induction homomorphism from $H=\left\langle s_{i}, s_{k}\right\rangle \cong C_{2} \times$ $C_{2}$ to $G=\left\langle s_{i}, s_{j}, s_{k}\right\rangle \cong \Delta(2,3,5)=A_{5} \times C_{2}$. 
Case $2: H=\left\langle s_{i}, s_{j}\right\rangle \cong D_{3}$

The characters of $G$ (Table 19) restricted to $H$ consists on the 1st, 8th, 8th, 2nd, 2nd and 8th columns:

\begin{tabular}{c|cccccc}
$A_{5} \times C_{2} \downarrow$ & $e$ & $s_{i}$ & $s_{j}$ & $s_{i} s_{j}$ & $s_{j} s_{i}$ & $s_{i} s_{j} s_{i}$ \\
\hline$\rho_{1} \otimes \xi_{1}$ & 1 & 1 & 1 & 1 & 1 & 1 \\
$\rho_{1} \otimes \xi_{2}$ & 4 & 0 & 0 & 1 & 1 & 0 \\
$\rho_{1} \otimes \xi_{3}$ & 5 & 1 & 1 & -1 & -1 & 1 \\
$\rho_{1} \otimes \xi_{4}$ & 3 & -1 & -1 & 0 & 0 & -1 \\
$\rho_{1} \otimes \xi_{5}$ & 3 & -1 & -1 & 0 & 0 & -1 \\
\hline$\rho_{2} \otimes \xi_{1}$ & 1 & -1 & -1 & 1 & 1 & -1 \\
$\rho_{2} \otimes \xi_{2}$ & 4 & 0 & 0 & 1 & 1 & 0 \\
$\rho_{2} \otimes \xi_{3}$ & 5 & -1 & -1 & -1 & -1 & -1 \\
$\rho_{2} \otimes \xi_{4}$ & 3 & 1 & 1 & 0 & 0 & 1 \\
$\rho_{2} \otimes \xi_{5}$ & 3 & 1 & 1 & 0 & 0 & 1
\end{tabular}

Multiplying these rows with the rows of the character table of $D_{3}$ (which is independent of whether $i<j$ or $j<i$ )

$$
\begin{array}{rll} 
& \rho_{1} \otimes_{-} & \rho_{2} \otimes_{-} \\
\chi_{1} \uparrow & =\xi_{1}+\xi_{2}+\xi_{3}+ & \xi_{2}+\xi_{4}+\xi_{5} \\
\chi_{2} \uparrow & =\xi_{2}+\xi_{4}+\xi_{5}+ & \xi_{1}+\xi_{2}+\xi_{3} \\
\phi_{1} \uparrow & =\xi_{2}+2 \xi_{3}+\xi_{4}+\xi_{5}+ & \xi_{2}+2 \xi_{3}+\xi_{4}+\xi_{5}
\end{array}
$$

This is then the map of free abelian groups shown in Figure 14.

$$
\begin{aligned}
R_{\mathbb{C}}(H) & \rightarrow R_{\mathbb{C}}(G) \\
\mathbb{Z}^{3} \rightarrow & \mathbb{Z}^{10} \\
(a, b, c) \rightarrow & (a, a+b+c, a+2 c, b+c, b+c \\
& b, a+b+c, b+2 c, a+c, a+c) .
\end{aligned}
$$

FIgURE 14. Induction homomorphism from $H=\left\langle s_{i}, s_{j}\right\rangle \cong D_{3}$ to $G=\left\langle s_{i}, s_{j}, s_{k}\right\rangle \cong \Delta(2,3,5)=A_{5} \times C_{2}$.

Case 3: $H=\left\langle s_{j}, s_{k}\right\rangle \cong D_{5}$

First we expand the character table for $D_{5}$ (from Table 4)

\begin{tabular}{c|cccccccccc}
$D_{5}$ & $e$ & $s_{j}$ & $s_{k}$ & $s_{j} s_{k}$ & $s_{k} s_{j}$ & $s_{j} s_{k} s_{j}$ & $s_{k} s_{j} s_{k}$ & $s_{j} s_{k} s_{j} s_{k}$ & $s_{k} s_{j} s_{k} s_{j}$ & $s_{j} s_{k} s_{j} s_{k} s_{j}$ \\
\hline$\chi_{1}$ & 1 & 1 & 1 & 1 & 1 & 1 & 1 & 1 & 1 & 1 \\
$\chi_{2}$ & 1 & -1 & -1 & 1 & 1 & -1 & -1 & 1 & 1 & -1 \\
$\phi_{1}$ & 2 & 0 & 0 & $\varphi-1$ & $\varphi-1$ & 0 & 0 & $\varphi$ & $\varphi$ & 0 \\
$\phi_{2}$ & 2 & 0 & 0 & $\varphi$ & $\varphi$ & 0 & 0 & $\varphi-1$ & $\varphi-1$ & 0
\end{tabular}

where $\varphi$ is the golden ratio $\frac{1+\sqrt{5}}{2}$, and we have used $2 \cos \left(\frac{2 \pi}{5}\right)=\varphi-1$ and $2 \cos \left(\frac{4 \pi}{5}\right)=\varphi$. Note that this table is independent of interchanging $s_{i}$ with $s_{j}$. Next we restrict the characters of $G$ (Table 19) to $H$, that is, the 1st, 8th, 8th, 4th, 


\begin{tabular}{|c|c|c|c|c|c|}
\hline$D_{2} \hookrightarrow A_{5} \times C_{2}$ & $(1)$ & $(12)(35) \alpha$ & 15) $(23) \alpha$ & \multicolumn{2}{|c|}{$(13)(25)$} \\
\hline$\beta_{1} \downarrow$ & 1 & 1 & 1 & \multicolumn{2}{|c|}{1} \\
\hline$\beta_{2} \downarrow$ & 0 & 0 & 0 & \multicolumn{2}{|c|}{0} \\
\hline$\beta_{3} \downarrow$ & 0 & 0 & 0 & \multicolumn{2}{|c|}{0} \\
\hline$\beta_{4} \downarrow$ & 0 & 0 & 0 & \multicolumn{2}{|c|}{0} \\
\hline$\beta_{5} \downarrow$ & 0 & 0 & 0 & \multicolumn{2}{|c|}{-4} \\
\hline$\beta_{6} \downarrow$ & 0 & -2 & -2 & \multicolumn{2}{|c|}{0} \\
\hline$\beta_{7} \downarrow$ & 0 & 0 & 0 & \multicolumn{2}{|c|}{0} \\
\hline$\beta_{8} \downarrow$ & 0 & 0 & 0 & \multirow{2}{*}{\multicolumn{2}{|c|}{$\begin{array}{l}0 \\
0\end{array}$}} \\
\hline$\beta_{9} \downarrow$ & 0 & 0 & 0 & & \\
\hline$\beta_{10} \downarrow$ & 0 & 0 & 0 & \multicolumn{2}{|c|}{0} \\
\hline$D_{2} \hookrightarrow A_{5} \times C_{2}$ & $\left(. \mid \sum \chi_{i}\right)$ & $\left(\cdot \mid \chi_{2}+\chi_{3}\right)$ & $\left(\cdot \mid \chi_{3}+\right.$ & $\left.\chi_{4}\right)$ & $\left(\cdot \mid \chi_{4}\right)$ \\
\hline$\beta_{1} \downarrow$ & 1 & 0 & 0 & & 0 \\
\hline$\beta_{2} \downarrow$ & 0 & 0 & 0 & & 0 \\
\hline$\beta_{3} \downarrow$ & 0 & 0 & 0 & & 0 \\
\hline$\beta_{4} \downarrow$ & 0 & 0 & 0 & & 0 \\
\hline$\beta_{5} \downarrow$ & 0 & 0 & 0 & & 1 \\
\hline$\beta_{6} \downarrow$ & 0 & 1 & 1 & & 0 \\
\hline$\beta_{7} \downarrow$ & 0 & 0 & 0 & & 0 \\
\hline$\beta_{8} \downarrow$ & 0 & 0 & 0 & & 0 \\
\hline$\beta_{9} \downarrow$ & 0 & 0 & 0 & & 0 \\
\hline$\beta_{10} \downarrow$ & 0 & 0 & 0 & & 0 \\
\hline
\end{tabular}

TABLE 34. Restricted characters (top) and map induced by the inclusion of $D_{2}$ into $A_{5} \times C_{2}$ (bottom).

4th, 8th, 8th, 5th, 5th and 8th columns.

\begin{tabular}{c|cccccccccc}
$A_{5} \times C_{2} \downarrow$ & $e$ & $s_{j}$ & $s_{k}$ & $s_{j} s_{k}$ & $s_{k} s_{j}$ & $s_{j} s_{k} s_{j}$ & $s_{k} s_{j} s_{k}$ & $s_{j} s_{k} s_{j} s_{k}$ & $s_{k} s_{j} s_{k} s_{j}$ & $s_{j} s_{k} s_{j} s_{k} s_{j}$ \\
\hline$\rho_{1} \otimes \xi_{1}$ & 1 & 1 & 1 & 1 & 1 & 1 & 1 & 1 & 1 & 1 \\
$\rho_{1} \otimes \xi_{2}$ & 4 & 0 & 0 & -1 & -1 & 0 & 0 & -1 & -1 & 0 \\
$\rho_{1} \otimes \xi_{3}$ & 5 & 1 & 1 & 0 & 0 & 1 & 1 & 0 & 0 & 1 \\
$\rho_{1} \otimes \xi_{4}$ & 3 & -1 & -1 & $\varphi$ & $\varphi$ & -1 & -1 & $-\varphi+1$ & $-\varphi+1$ & -1 \\
$\rho_{1} \otimes \xi_{5}$ & 3 & -1 & -1 & $-\varphi+1$ & $-\varphi+1$ & -1 & -1 & $\varphi$ & $\varphi$ & -1 \\
\hline$\rho_{2} \otimes \xi_{1}$ & 1 & -1 & -1 & 1 & 1 & -1 & -1 & 1 & 1 & -1 \\
$\rho_{2} \otimes \xi_{2}$ & 4 & 0 & 0 & -1 & -1 & 0 & 0 & -1 & -1 & 0 \\
$\rho_{2} \otimes \xi_{3}$ & 5 & -1 & -1 & 0 & 0 & -1 & -1 & 0 & 0 & -1 \\
$\rho_{2} \otimes \xi_{4}$ & 3 & 1 & 1 & $\varphi$ & $\varphi$ & 1 & 1 & $-\varphi+1$ & $-\varphi+1$ & 1 \\
$\rho_{2} \otimes \xi_{5}$ & 3 & 1 & 1 & $-\varphi+1$ & $-\varphi+1$ & 1 & 1 & $\varphi$ & $\varphi$ & 1
\end{tabular}

Remark 6. With a non-conjugated choice of Coxeter generators, it would be the 5 th instead of the 4 th column, or, equivalently, swapping $\rho_{i} \otimes \xi_{4}$ with $\rho_{i} \otimes \xi_{5}$ for $i=1$ and 2 .

Multiplying these rows with the rows of character table of $D_{5}$ above, and using that $\varphi^{2}-\varphi=1$, we obtain

$$
\begin{array}{rll} 
& \rho_{1} \otimes_{-} & \rho_{2} \otimes_{-} \\
\chi_{1} \uparrow & =\xi_{1}+\xi_{3}+ & \xi_{4}+\xi_{5} \\
\chi_{2} \uparrow & =\xi_{4}+\xi_{5}+ & \xi_{1}+\xi_{3} \\
\phi_{1} \uparrow & =\xi_{2}+\xi_{3}+\xi_{4}+ & \xi_{2}+\xi_{3}+\xi_{4} \\
\phi_{2} \uparrow & =\xi_{2}+\xi_{3}+\xi_{5}+ & \xi_{2}+\xi_{3}+\xi_{5}
\end{array}
$$

This gives the homomorphism of free abelian groups in Figure 15 .

We finish by giving the same induction homomorphisms but this time with respect to the transformed bases (Tables 6, 7, 20 in Appendix A) in Tables 34, 35 and 36 . 


$$
\begin{aligned}
& R_{\mathbb{C}}(H) \rightarrow R_{\mathbb{C}}(G) \\
& \mathbb{Z}^{4} \rightarrow \mathbb{Z}^{10} \\
& (a, b, c, d) \mapsto \quad(a, c+d, a+c+d, b+c, b+d, \\
& b, c+d, b+c+d, a+c, a+d) .
\end{aligned}
$$

FiguRE 15. Induction homomorphism from $H=\left\langle s_{j}, s_{k}\right\rangle \cong D_{5}$ to $G=\left\langle s_{i}, s_{j}, s_{k}\right\rangle \cong \Delta(2,3,5)=A_{5} \times C_{2}$.

\begin{tabular}{|c|c|c|c|c|c|c|c|c|}
\hline$D_{5} \hookrightarrow A_{5} \times C_{2}$ & (1) & $(12345)$ & (12354) & $(12)(34) \alpha$ & $\left(\cdot \mid \sum \chi_{i}+2 \sum \phi_{i}\right)$ & $\left(\cdot \mid \chi_{2}+\sum \phi_{i}\right)$ & $\left(\cdot \mid \sum \phi_{i}\right)$ & $\left(\cdot \mid \phi_{2}\right)$ \\
\hline $\bar{\beta} \beta_{1} \downarrow$ & 1 & 1 & 1 & 1 & 1 & 0 & 0 & 0 \\
\hline$\beta_{2} \downarrow$ & 0 & $\sqrt{5}$ & $-\sqrt{5}$ & 0 & 0 & 0 & 0 & 1 \\
\hline$\beta_{3} \downarrow$ & 0 & 0 & 0 & 0 & 0 & 0 & 0 & 0 \\
\hline$\beta_{4} \downarrow$ & 0 & $\frac{5+\sqrt{5}}{2}$ & $\frac{5-\sqrt{5}}{2}$ & 0 & 0 & 0 & -1 & 0 \\
\hline$\beta_{5} \downarrow$ & 0 & ${ }_{0}^{2}$ & $0_{0}^{2}$ & 0 & 0 & 0 & 0 & 0 \\
\hline$\beta_{6} \downarrow$ & 0 & 0 & 0 & -2 & 0 & 1 & 0 & 0 \\
\hline$\beta_{7} \downarrow$ & 0 & 0 & 0 & 0 & 0 & 0 & 0 & 0 \\
\hline$\beta_{8} \downarrow$ & 0 & 0 & 0 & 0 & 0 & 0 & 0 & 0 \\
\hline$\beta_{9} \downarrow$ & 0 & 0 & 0 & 0 & 0 & 0 & 0 & 0 \\
\hline$\beta_{10} \downarrow$ & 0 & 0 & 0 & 0 & 0 & 0 & 0 & 0 \\
\hline
\end{tabular}

\begin{tabular}{c|ccc||ccc}
$D_{3} \hookrightarrow A_{5} \times C_{2}$ & $(1)$ & $(12)(34) \alpha$ & $(123)$ & $\left(\cdot \mid \sum \chi_{i}+2 \phi_{1}\right)$ & $\left(\cdot \mid \chi_{2}+\phi_{1}\right)$ & $\left(\cdot \mid \phi_{1}\right)$ \\
\hline$\beta_{1} \downarrow$ & 1 & 1 & 1 & 0 & 0 & 0 \\
$\beta_{2} \downarrow$ & 0 & 0 & 0 & 0 & 0 & 0 \\
$\beta_{3} \downarrow$ & 0 & 0 & -3 & 0 & 0 & 1 \\
$\beta_{4} \downarrow$ & 0 & 0 & 0 & 0 & 0 & 0 \\
$\beta_{5} \downarrow$ & 0 & 0 & 0 & 0 & 0 & 0 \\
\hline$\beta_{6} \downarrow$ & 0 & -2 & 0 & 0 & 1 & 0 \\
$\beta_{7} \downarrow$ & 0 & 0 & 0 & 0 & 0 & 0 \\
$\beta_{8} \downarrow$ & 0 & 0 & 0 & 0 & 0 & 0 \\
$\beta_{9} \downarrow$ & 0 & 0 & 0 & 0 & 0 & 0 \\
$\beta_{10} \downarrow$ & 0 & 0 & 0 & 0 & 0 & 0
\end{tabular}

TABLE 35. Restricted characters and map induced by the inclusion of $D_{3}$ into $A_{5} \times C_{2}$.

TABLE 36. Restricted characters and map induced by the inclusion of $D_{5}$ into $A_{5} \times C_{2}$.

We show that $\mathrm{H}_{0}^{\mathfrak{F i n}}\left(\Gamma ; R_{\mathbb{C}}\right)$ is torsion-free for $\Gamma$ the Heisenberg semidirect product group of Lück's paper.

\section{Appendix C. The Heisenberg semidirect product group}

In Tables 37 and 38, we transform the character tables of all the non-trivial finite subgroups of the Heisenberg semidirect product group, as specified by Lück [14].

In Tables 39, 40 and 41, we compute all possible induction homomorphisms $R_{\mathbb{C}}(H) \rightarrow R_{\mathbb{C}}(G)$ appearing in any possible Bredon chain complex. 
Cyclic group of order 2 with generator $s$.

$$
\left(\begin{array}{c|rr}
C_{2} & e & s \\
\hline \rho_{1} & 1 & 1 \\
\rho_{2} & 1 & -1
\end{array}\right) \mapsto\left(\begin{array}{c|rr}
C_{2} & e & s \\
\hline \rho_{1}+\rho_{2} & 2 & 0 \\
\rho_{2} & 1 & -1
\end{array}\right)
$$

TABLE 37. Character table of $C_{2}$

Cyclic group of order 4 with generator $s$, we let $i^{2}=-1$.

$$
\left(\begin{array}{r|rrrr}
C_{4} & e & s & s^{2} & s^{3} \\
\hline \rho_{1} & 1 & 1 & 1 & 1 \\
\rho_{2} & 1 & -1 & 1 & -1 \\
\rho_{3} & 1 & i & -1 & -i \\
\rho_{4} & 1 & -i & -1 & i
\end{array}\right) \mapsto\left(\begin{array}{c|rrrr}
C_{4} & e & s & s^{2} & s^{3} \\
\hline \rho_{1} & 1 & 1 & 1 & 1 \\
\rho_{2}-\rho_{1} & 0 & -2 & 0 & -2 \\
\rho_{3}-\rho_{1} & 0 & i-1 & -2 & -i-1 \\
\rho_{4}-\rho_{3} & 0 & -2 i & 0 & 2 i
\end{array}\right)
$$

TABle 38. Character table of $C_{4}$

The only non-trivial inclusion of a cyclic group of order 2 into a cyclic group of order 4:

\begin{tabular}{c|rr|cc}
$C_{2} \hookrightarrow C_{4}$ & $e$ & $s^{2}$ & $\left(\cdot \mid \rho_{1}+\rho_{2}\right)$ & $\left(\cdot \mid \rho_{2}\right)$ \\
\hline$\rho_{1} \downarrow$ & 1 & 1 & 1 & 0 \\
$\left(\rho_{2}-\rho_{1}\right) \downarrow$ & 0 & 0 & 0 & 0 \\
$\left(\rho_{3}-\rho_{1}\right) \downarrow$ & 0 & -2 & 0 & 1 \\
$\left(\rho_{4}+\rho_{3}-\rho_{2}-\rho_{1}\right) \downarrow$ & 0 & 0 & 0 & 0
\end{tabular}

TABLE 39. The only non-trivial inclusion $C_{2} \hookrightarrow C_{4}: s \mapsto s^{2}$.

The trivial inclusion of a cyclic group of order 2 into a cyclic group of order 4:

\begin{tabular}{c|cc|cc}
$C_{2} \hookrightarrow C_{4}$ & $e$ & $e$ & $\left(\cdot \mid \rho_{1}+\rho_{2}\right)$ & $\left(\cdot \mid \rho_{2}\right)$ \\
\hline$\rho_{1} \downarrow$ & 1 & 1 & 1 & 0 \\
$\left(\rho_{2}-\rho_{1}\right) \downarrow$ & 0 & 0 & 0 & 0 \\
$\left(\rho_{3}-\rho_{1}\right) \downarrow$ & 0 & 0 & 0 & 0 \\
$\left(\rho_{4}+\rho_{3}-\rho_{2}-\rho_{1}\right) \downarrow$ & 0 & 0 & 0 & 0
\end{tabular}

TABLE 40. The trivial inclusion $C_{2} \hookrightarrow C_{4}: s \mapsto e$.

The inclusion of the trivial group into a cyclic group of order 4:

\begin{tabular}{c|c|c}
$C_{2} \hookrightarrow C_{4}$ & $e$ & $(\cdot \mid \tau)$ \\
\hline$\rho_{1} \downarrow$ & 1 & 1 \\
$\left(\rho_{2}-\rho_{1}\right) \downarrow$ & 0 & 0 \\
$\left(\rho_{3}-\rho_{1}\right) \downarrow$ & 0 & 0 \\
$\left(\rho_{4}+\rho_{3}-\rho_{2}-\rho_{1}\right) \downarrow$ & 0 & 0
\end{tabular}

TABLE 41. The inclusion of the trivial group $\{1\} \hookrightarrow C_{4}$

Obviously, any concatenation of copies of the three matrices given in Tables 39, 40 and 41 yields a matrix with all of its minors contained in the set $\{-1,0,1\}$. For the inclusions into cyclic groups of order 2, we proceed analogously, only it is then 
even simpler to compute the induced matrices. Hence by Theorem $4, \mathrm{H}_{0}^{\mathfrak{F i n}}\left(\Gamma ; R_{\mathbb{C}}\right)$ is torsion-free for $\Gamma$ the Heisenberg semidirect product group of Lück's article [14].

\section{Appendix D. Crystallographic groups}

Davis and Lück [4] consider the semidirect product of $\mathbb{Z}^{n}$ with the cyclic $p$ group $\mathbb{Z} / p$, where the action of $\mathbb{Z} / p$ on $\mathbb{Z}^{n}$ is given by an integral representation, which is assumed to act freely on the complement of zero. The action of this semidirect product group $\Gamma$ on $\underline{\mathrm{E}} \Gamma \cong \mathbb{R}^{n}$ is crystallographic, with $\mathbb{Z}^{n}$ acting by lattice translations, and $\mathbb{Z} / p$ acting with a single fixed point. In particular, all cell stabilizers are trivial except for one orbit of vertices of stabilizer type $\mathbb{Z} / p$. So all maps in the Bredon chain complex are induced by the trivial representation, and we can easily apply Theorem 4 to see that $\mathrm{H}_{0}^{\mathfrak{F i n}}\left(\Gamma ; R_{\mathbb{C}}\right)$ is torsion-free for $\Gamma$. 